RAIDAR - ES\&T Supporting Information (es0514085)

Screening level risk assessment model for chemical fate and effects in the environment Supporting Information

\author{
Jon A. Arnot* \\ Don Mackay \\ Eva Webster \\ Canadian Environmental Modelling Centre \\ 1600 West Bank Drive, Trent University \\ Peterborough, ON \\ K9J 7B8 \\ Jeanette M. Southwood \\ Golder Associates, \\ 32 Steacie Drive \\ Kanata, ON \\ K2K 2 A9 \\ Contact information: \\ jonarnot@trentu.ca \\ T 705-748-1011 extn 1645 \\ F 705-748-1080
}

Number of pages: $\quad 41$

Number of tables: $\quad 14$

Number of Figures: 3 
RAIDAR - ES\&T Supporting Information (es0514085)

\section{Evaluative environment}

The purpose of the model is to provide screening level risk assessments of chemicals in a consistent evaluative environment. Table SI-1 lists the abiotic and biotic components of the RAIDAR evaluative environment. Tables SI-2 and SI-3 respectively summarize key parameters of the abiotic and biotic (food webs) compartments. Fate and transport of chemical in the abiotic environment are determined by Level II and Level III fugacity model calculations described elsewhere $[1,2]$ except where otherwise noted. Representative food webs are included to assess chemical exposure routes from the environment to ecological and human receptors. The bioaccumulation of chemical in fish, wildlife, agriculture and humans is quantified using a generic bioaccumulation model that is subsequently described.

\section{Chemical concentration and fugacity}

Chemical concentrations $\mathrm{C}\left(\mathrm{mol} / \mathrm{m}^{3}\right)$ are expressed as the product $\mathrm{Z} f$ where $f(\mathrm{~Pa})$ is the fugacity and $\mathrm{Z}\left(\mathrm{mol} / \mathrm{m}^{3} \cdot \mathrm{Pa}\right)$ is the fugacity capacity. The fugacity capacity of a phase depends on the chemical and the nature and temperature of the medium or compartment. $Z$ values quantify the ability of a phase to retain a chemical. The fugacity can be regarded as a partial pressure or the "escaping tendency" of a particular chemical in a particular phase. Chemical uptake and loss processes are defined by a D value $(\mathrm{mol} / \mathrm{Pa} \cdot \mathrm{h})$, which is essentially a contaminant transport or transformation rate parameter. The rate of chemical transport or flux $\mathrm{N}(\mathrm{mol} / \mathrm{h})$ is the product $\mathrm{D} f$. It follows that larger D values refer to faster contaminant transport processes. These $\mathrm{D}$ values are analogous to rate constants. Tables SI-4 and SI-5 provide definitions for some of these parameters while full details of the fugacity concept are explained elsewhere [1].

\section{Fugacity-based generic bioaccumulation model and representative food webs}

Bioaccumulation is the result of the competing processes of chemical uptake and elimination in an organism. Figure SI-1 illustrates a conceptual model for major routes of uptake and elimination in aquatic and terrestrial organisms. Assuming steady-state conditions, a general mass balance expression written in terms of the flux of chemical into and out of an organism is:

$$
\underset{\text { uptake }}{\mathrm{D}_{\mathrm{V}} f_{\mathrm{V}}+\sum \mathrm{D}_{\mathrm{D}, \mathrm{i}, \mathrm{i}} f_{\mathrm{W}}}+\mathrm{D}_{\mathrm{W}} f_{\mathrm{W}}=f_{\mathrm{B}}\left(\mathrm{D}_{\mathrm{V}}+\mathrm{D}_{\mathrm{E}}+\mathrm{D}_{\mathrm{G}}+\mathrm{D}_{\mathrm{M}}+\mathrm{D}_{\mathrm{U}}+\mathrm{D}_{\mathrm{O}}+\mathrm{D}_{\mathrm{L}}\right)
$$

where $D_{V}, D_{D}, D_{W}, D_{E}, D_{G}, D_{M}, D_{U}, D_{O}, D_{L}$ represent respiration or ventilation (i.e., uptake from and loss to air or water), uptake from ingestion of food, uptake from ingestion of water, egestion in feces, pseudo-elimination by "growth dilution", loss from metabolism of parent compound, urinary excretion, off-spring losses and lactation $\mathrm{D}$ values, respectively. $f_{\mathrm{V}}$ is the fugacity in respired air or water, $f_{\mathrm{D}}$ is the fugacity in the diet, $f_{\mathrm{W}}$ is the fugacity in drinking water and $f_{\mathrm{B}}$ is the fugacity in the organism. i represents each of the individual dietary components of an organism. Dermal uptake and elimination rates are not considered in the model. The rate of fecal egestion can be estimated as:

$$
\mathrm{D}_{\mathrm{E}} f_{\mathrm{B}}=\Sigma \mathrm{D}_{\mathrm{D}, \mathrm{i}} f_{\mathrm{D}, \mathrm{i}} / \mathrm{Q}(2)
$$


where $\mathrm{Q}$ is the species-specific theoretical maximum biomagnification factor representing biotafeces partitioning. The value varies between species because it is dependent on specific factors related to the digestion process as a result of dietary preferences, the digestibility of the diet and the physiology of the organism. Essentially, Q is a product of a reduction in the volume of diet as it passes through the gastrointestinal tract and an elevation of the biota-to-gut partition coefficient above the biota-to-diet partition coefficient [3]. Q is lower in herbivorous organisms than carnivorous organisms. Water ingestion for fish is considered insignificant compared to chemical uptake at the gill and urinary excretion is similarly excluded for fish. The algorithms for each bioaccumulation D value are included in Table SI-5.

Equation 1 is used to assess bioaccumulation in a range of species with a few exceptions. The fugacities calculated for plankton, vegetation and aquatic and terrestrial invertebrates are assumed to be a result of thermodynamic equilibrium with the media in which they reside. Specifically, plankton is at equilibrium with the water, benthic invertebrates are at equilibrium with the sediment and terrestrial invertebrates are at equilibrium with the soil. Likewise, root vegetation is at equilibrium with the soil whereas foliage vegetation is at equilibrium with the air. Other organisms, including humans, are represented by the general bioaccumulation expression. Fish ventilate water while all other organisms respire air.

This model includes the evaluation of hydrophobic chemicals as well as more watersoluble chemicals and ionizing compounds and a three compartment partitioning model is included. In addition to the lipid and water fractions, nonlipid organic matter (NLOM), such as proteins in animals $[4,5]$ and carbohydrates in plants [6], also play a role in partitioning. Thus, the total storage capacity (i.e. Z) for plants and animals in the model is a reflection of the relative contributions of each component as:

$$
\mathrm{Z}_{\mathrm{B}}=v_{\mathrm{L}} \mathrm{Z}_{\mathrm{L}}+v_{\mathrm{NLOM}} \mathrm{Z}_{\mathrm{NLOM}}+v_{\mathrm{W}} \mathrm{Z}_{\mathrm{WT}}(3)
$$

where $Z_{\mathrm{B}}, Z_{\mathrm{L}}, \mathrm{Z}_{\mathrm{NLOM}}, \mathrm{Z}_{\mathrm{WT}}$ are the fugacity capacities of the biota, lipid, NLOM and water, respectively and $v_{\mathrm{L}}, v_{\mathrm{NLOM}}, v_{\mathrm{W}}$ are the volume fractions of the component parts.

Table SI-6 provides a summary of the allometric relationships used to describe the specific rates of air inhalation $G_{V}$, feeding $G_{D}$ and water intake $G_{W}$ for all avian and mammalian species [7] and for feeding $G_{D}$ and water ventilation $G_{V}$ for fish [4]. For the allometric feeding rates, a wet weight:dry weight conversion ratio $\left(\mathrm{R}_{\mathrm{WD}}\right)$ of $4: 1$ is assumed for all dietary items (except "poultry" where 2:1 is used). The conversions are not required for fish as the feeding rate is derived for dietary wet weight. The majority of the allometric relationships describe rate processes as L or $\mathrm{kg}$ per day and are converted to $\mathrm{m}^{3} / \mathrm{h}$ for the model. The whole body density of all organisms is assumed to be $1 \mathrm{~kg} / \mathrm{L} . \mathrm{E}_{\mathrm{V}}$, the environmental medium chemical transfer efficiency, for mammals and birds is assumed to be 1 , whereas for fish it is described in Table SI$6[8]$.

The efficiency of chemical uptake from the diet $E_{D}$ is observed to be greatest in mammals and birds while efficiency is less in fish $[3,4,9-11] . \mathrm{E}_{\mathrm{D}}$ is a function of the hydrophobicity of the 
chemical and is observed to decrease for chemicals with log $\mathrm{K}_{\mathrm{OW}} \sim 6-7$ and greater in fish and slightly higher in these other species. For fish this equation is:

$$
\mathrm{E}_{\mathrm{D}}=\left(2.0+10^{-8} \mathrm{~K}_{\mathrm{OW}}\right)^{-1}(4)
$$

and for avian species and mammals:

$$
\mathrm{E}_{\mathrm{D}}=\left(1.05+10^{-10} \mathrm{~K}_{\mathrm{OW}}\right)^{-1}(5)
$$

Bulk dairy (e.g., milk, cheese, yogurt, etc) and chicken eggs are dietary items included in most human diets. The capacity of these media to store chemical are estimated from Equation 3 and parameters from Table SI-3. Estimated chemical concentrations in these agricultural products and others (i.e., meat) are assumed to be the result of equilibrium partitioning within the organism.

The chemical solubility in water and octanol-water partition coefficient for the neutral or non-ionic species are required as inputs for the RAIDAR model. A correction for ionization is included in the model for substances that can dissociate in water. An acid with a low pKa (e.g., 3) ionizes appreciably as does a base with a high $\mathrm{pKb}$ (e.g., 11). In the interest of simplicity the bases are treated using a $\mathrm{pKa}$ equation. The same equation is thus applied to bases since they are also characterized by $\mathrm{pKa}$. When an organic acid ionizes the ratio of ionized (I) to non ionized (NI) is given by $\mathrm{I} / \mathrm{NI}=10^{(\mathrm{pH}-\mathrm{pKa})}$.

The fugacity capacity for water for the ionized fraction of dissociating chemicals can be calculated as $Z_{\mathrm{WI}}\left(\right.$ ionic) $=\mathrm{Z}_{\mathrm{WN}}$ (neutral) $\bullet \mathrm{I} / \mathrm{NI}$ and $\mathrm{Z}_{\mathrm{WT}}$ (total) $=\mathrm{Z}_{\mathrm{WN}}+\mathrm{Z}_{\mathrm{WI}}$. The RAIDAR model assumes that the $\mathrm{K}_{\mathrm{OW}}$ for a dissociating chemical is that of the neutral species. For this case study, the model assumes internal organism and fish gill $\mathrm{pH}$ to be the same as the environment (i.e., 7). The current approach provides a reasonable first approximation and may be refined as the science progresses.

\section{Food Web Model Parameterization}

Table SI-3 illustrates respiration, feeding, drinking, growth and urination rates derived from the equations described in Tables SI-5 and SI-6. These rates are generally comparable with empirical food web studies and evaluated models for persistent organic pollutants (POPs) [3, 4, 9, 10, 12]. The theoretical maximum biomagnification factors, lipid contents, wet weights and feeding preferences were based on these empirical and evaluated studies. Feeding preferences used in this evaluation are presented in Table SI-7. The model is parameterized for "adult male" organisms such that reproductive and lactation losses are not included. This allows for conservative estimates since females are known to lower their chemical body burdens of hydrophobic chemicals through these mechanisms. Thus, for screening level evaluations it is assumed that $1 \%$ of the diet is converted to new body mass as a 'growth rate' to calculate growth dilution in Equation 1. For all organisms that drink water, the urinary excretion rate was assumed to be equal to the water intake rate. It was assumed that protein and carbohydrate (i.e., $v_{\text {NLOM) }}$ contribute approximately $20 \%$ to most biota. 
The model allows for the input of metabolic transformation rates for chemicals that are known to be metabolized. There is a lack of data available for the metabolic transformation rate of most chemicals and this parameter is highly variable and uncertain in organisms. Thus, it was assumed as a default to be negligible. This practice of ignoring metabolism is commonly used by regulatory agencies, i.e., in the absence of information to the contrary assume no metabolism. This conservative approach is considered reasonable for assessing risk at a screening level and the current level of knowledge on rates of metabolic transformation.

In the RAIDAR model the representative food webs are kept relatively simple but are still indicative of major exposure routes to key ecological receptors and to humans. The exposure pathways and food webs could be manipulated for specific ecosystems (e.g., number of organisms included and their characteristics) and for humans to reflect different demographics and subpopulations. Currently, the RAIDAR model accounts for "farfield" exposures and does not calculate occupational or industrial exposures or indoor air and consumer product uses (e.g., personal care or household).

\section{Other notable RAIDAR model calculations}

There are some differences in the RAIDAR model from Level II and Level III calculations found in the EQC model [1]. Notably the process D-value for rain dissolution $\left(\mathrm{D}_{\mathrm{R}}\right)$ is calculated based on the relationship between $\mathrm{K}_{\mathrm{AW}}$ and the scavenging efficiency of rain as

$$
\begin{aligned}
& \mathrm{D}_{\mathrm{R}}=\mathrm{A} \times \mathrm{MTC}_{\mathrm{Rain}} \times \mathrm{Z}_{\mathrm{WN}} \text { for } \mathrm{K}_{\mathrm{AW}} \geq 1 / \text { Scavenging (6) } \\
& \mathrm{D}_{\mathrm{R}}=\mathrm{A} \times \mathrm{MTC}_{\mathrm{Rain}} \times \mathrm{Scavenging} \times \mathrm{Z}_{\mathrm{A}} \text { for } \mathrm{K}_{\mathrm{AW}}<1 / \text { Scavenging (7) }
\end{aligned}
$$

Where A is the surface area of either water or soil. The Level III model version 2.80 uses equation 6 for all chemicals. Equation 7 limits removal by rain dissolution by setting a maximum value for low $\mathrm{K}_{\mathrm{AW}}$ chemicals.

Partitioning to aerosols in the RAIDAR environment is approximated by a proportionality constant of 0.1 as $\mathrm{K}_{\mathrm{QA}}=0.1 \cdot \mathrm{K}_{\mathrm{OA}}$. This assumes that particles contain about $20 \%$ organic matter with an "octanol" equivalence of approximately $10 \%$.

The advective flow residence time for water in the evaluative EQC environment is increased from $10^{4}$ to $10^{5}$ hours as the residence time for water in the standard EQC environment was originally intended to reflect the rapid flushing form a large volume of water as occurs in regions such as the North American Great Lakes. The revised longer residence time is more generally applicable.

As in EQC, to ensure internally consistent partitioning behaviour in the RAIDAR model the octanol-air partition coefficient is calculated from the octanol-water partition coefficient and the unitless air-water partition coefficient as $\mathrm{K}_{\mathrm{OA}}=\mathrm{K}_{\mathrm{OW}} / \mathrm{K}_{\mathrm{Aw}}$, where $\mathrm{K}_{\mathrm{Aw}}$ is $\mathrm{H} / \mathrm{RT}$. 
RAIDAR - ES\&T Supporting Information (es0514085)

\section{Characteristic Transport}

A characteristic travel distance for long range transport in air $\left(L_{\mathrm{A}}\right)$ is calculated for each chemical as $U \cdot F_{A} \cdot \tau_{R}$ where $U$ is an assumed air velocity of $4 \mathrm{~m} / \mathrm{s}$ and $F_{A}$ is fraction of chemical mass in air [13].

\section{Illustrative model output}

Illustrative model output referred to in the manuscript is presented. Table SI- 8 includes unit concentrations $\left(\mathrm{C}_{\mathrm{U}}\right)$ in all abiotic and biotic concentrations for five different model scenarios based on a unit emission rate $\left(\mathrm{E}_{\mathrm{U}}\right)$ of $1000 \mathrm{~kg} / \mathrm{h}(1 \mathrm{t} / \mathrm{h})$. Table SI-8 indicates the most sensitive endpoints for each model scenario in bold font for "objective" endpoints and bold italic font for "effect" endpoints. Examples of RAIDAR calculated hazard data are also presented in Table SI-8 (e.g., BAF, $\left.P_{\mathrm{OV}}, L_{\mathrm{A}}\right)$.

Figure SI-2 illustrates risk quotients (RQ) for each RAIDAR compartment based on "objective" and "effect" endpoints for B(a)P, DCE and HCB from unit emissions in Level II and Level III calculations.

Table SI-9 lists RAIDAR model input for 70 illustrative chemicals described in the manuscript. Results including the most sensitive endpoint, critical emission rate, RAF, risk identification "bin" and relative rank are summarized for the Level II and each of the four Level III model scenarios are summarized in Tables SI-10 through SI-14.

Figure SI-3 compares different Level III mode of entry RAFs to Level II RAFs. This indicates the selection of fate calculation does not have a large impact on the overall rankings. In absence of reliable information the more "simple" Level II calculation may be sufficient for screening purposes.

\section{Actual emission rate estimation for 70 chemicals}

Emission rates were estimated from Canada's National Pollutant Release Inventory where they were available [14]. If they were not available they were estimated from annual production quantity range data reported for the DSL (ca. 1986) [15]. If the quantity was expressed as a range between 1 and 1,000 tonnes the geometric mean was chosen as an estimate of annual emissions (i.e. $31.6 \mathrm{t} /$ year). If the quantity was greater than or equal to 1000 tonnes a value of 3,160 tonnes was selected. If the quantity was less than 1 tonne an emission rate of $316 \mathrm{~kg} /$ year was estimated. Estimated emissions rates are from reported "point sources" (i.e., industrial) and do not include releases from "non-point sources" such as vehicle exhaust (i.e., PAHs) or leachate from consumer products (i.e., phthalates, PBDEs) and landfills. Actual emission rate $\left(\mathrm{E}_{\mathrm{A}}\right)$ estimates are included to illustrate the method. More recent and reliable data are essential for accurately determining RAFs from the model. 

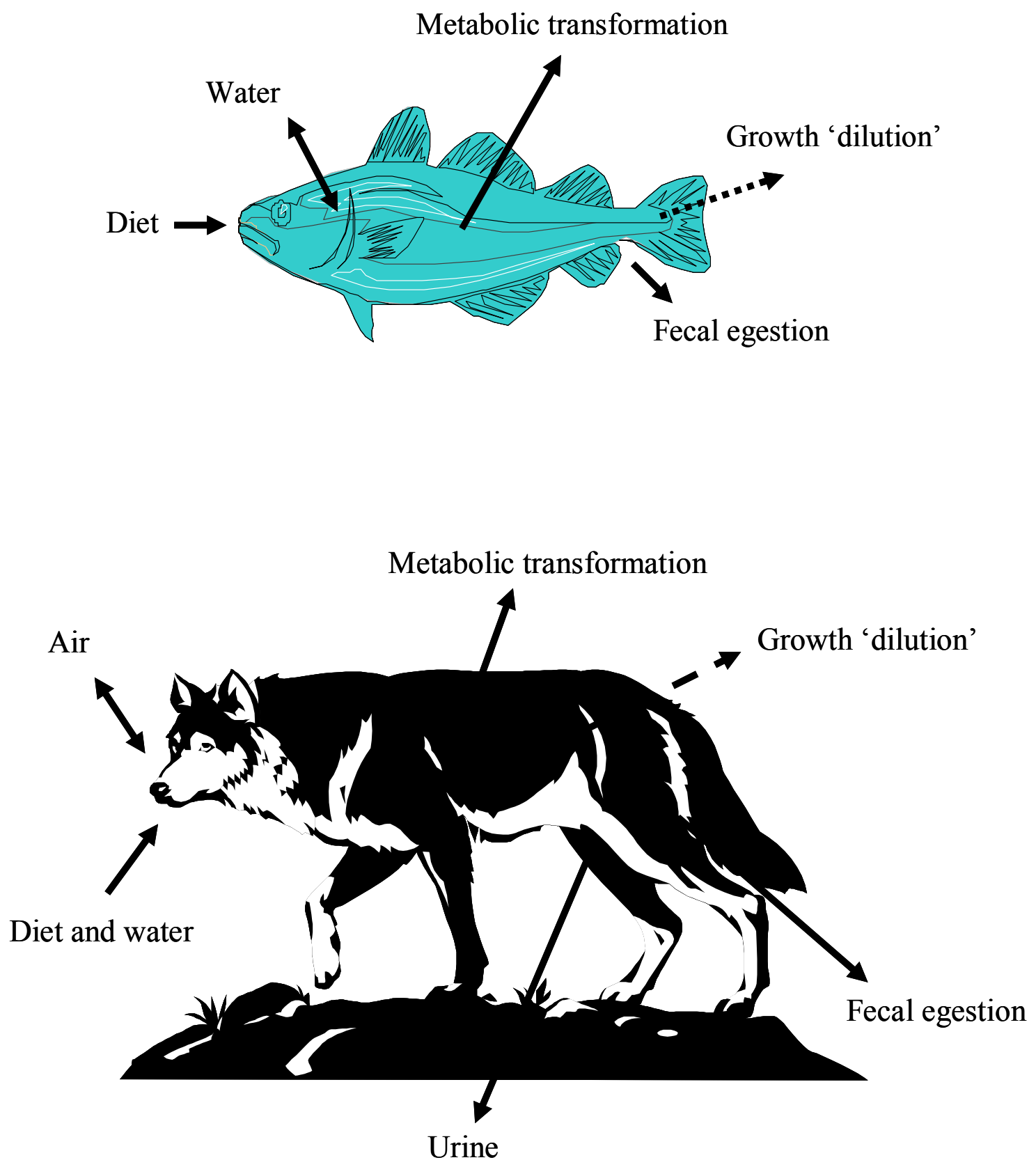

Figure SI - 1. Conceptual overview for major routes of chemical uptake and elimination included in the general bioaccumulation model. 


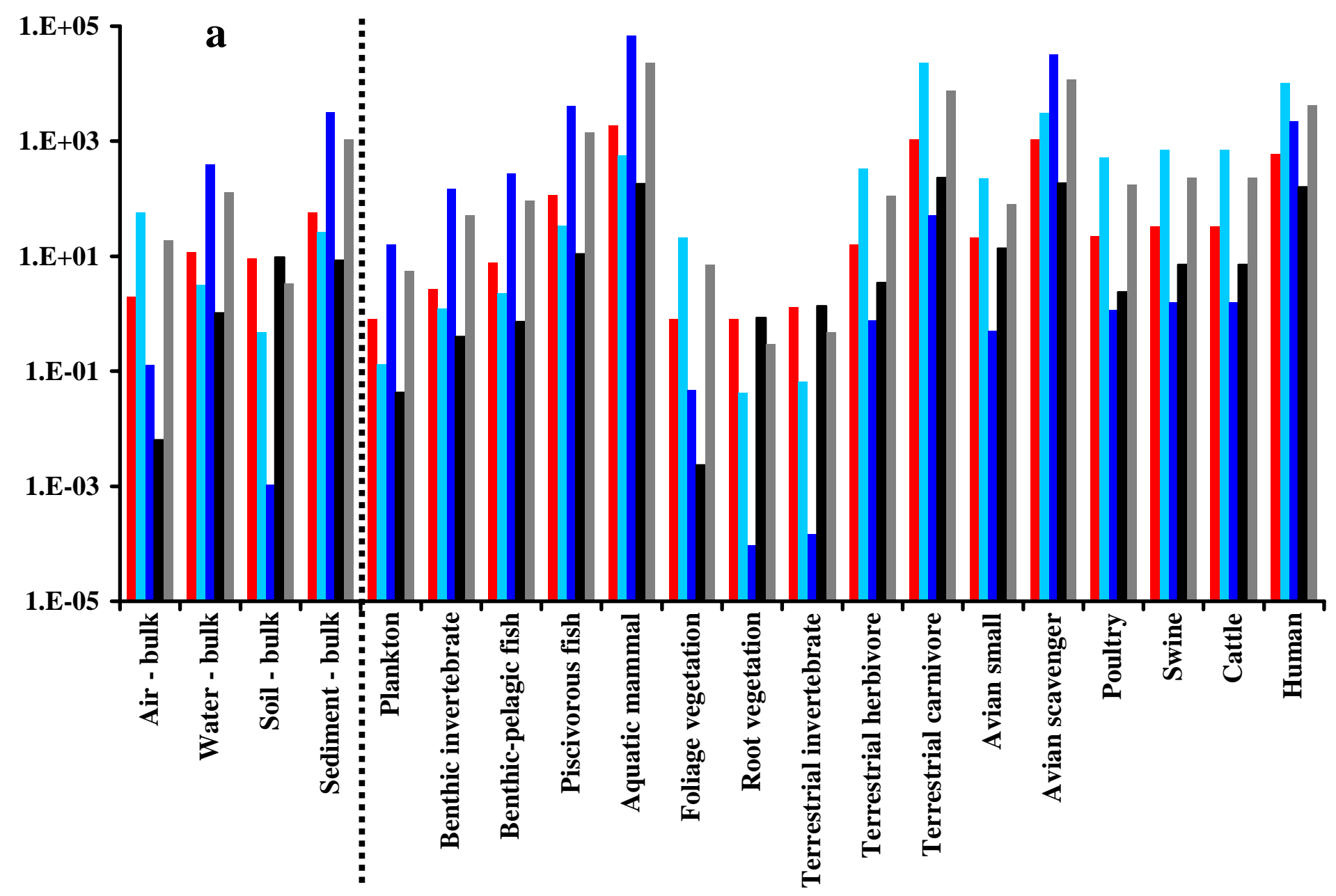




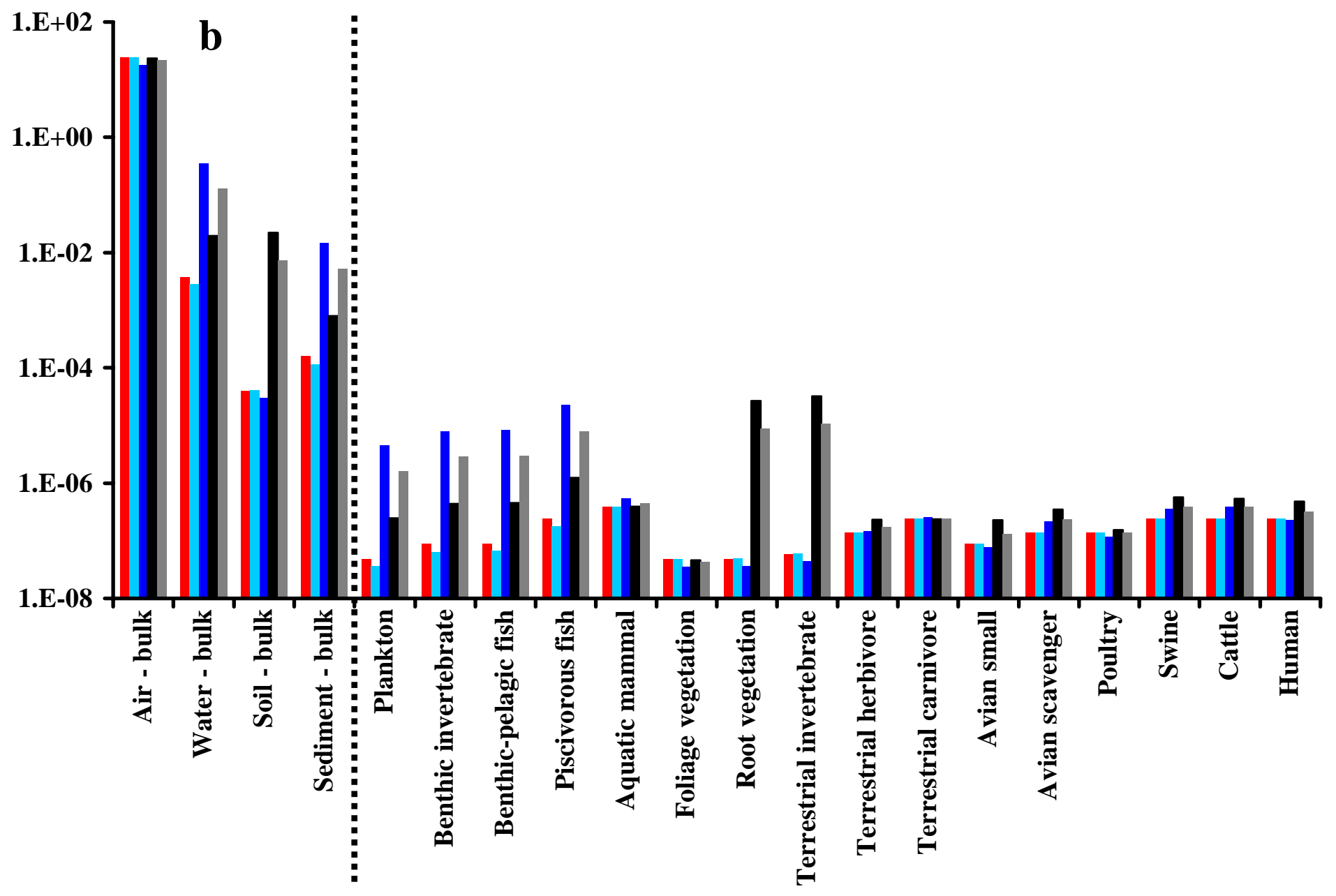




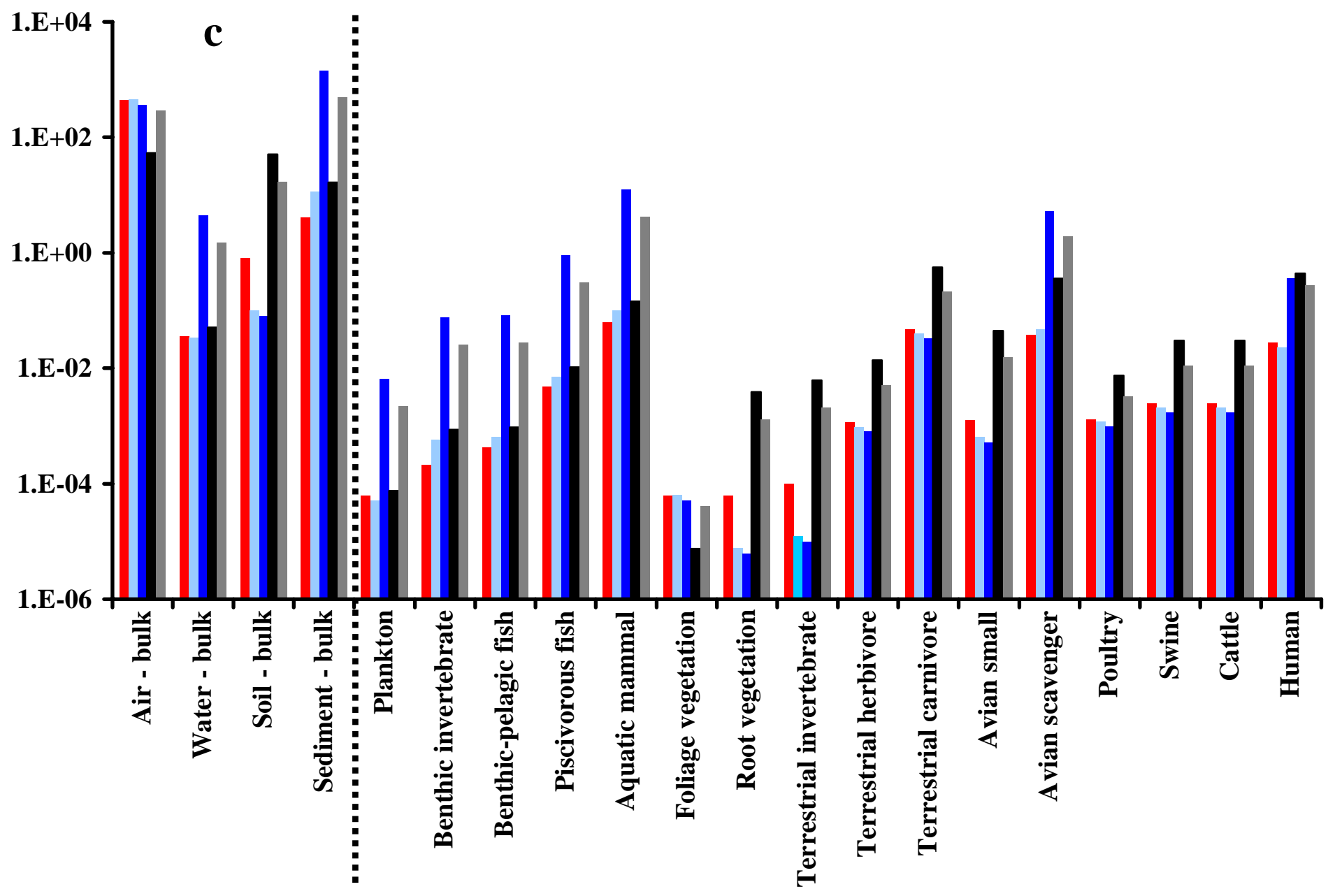

Figure SI-2. Risk quotients in compartments of the RAIDAR model for "objective" (left of dashed line) and "effect" (right of dashed line) endpoints (a - B(a)P; b - DCE; c - HCB). Model scenarios: Red - Level II; light blue - Level III (MOE-air); dark blue - Level III (MOE-water); black - Level III (MOE-soil); grey - Level III (MOE-AWS). 
RAIDAR - ES\&T Supporting Information (es0514085)
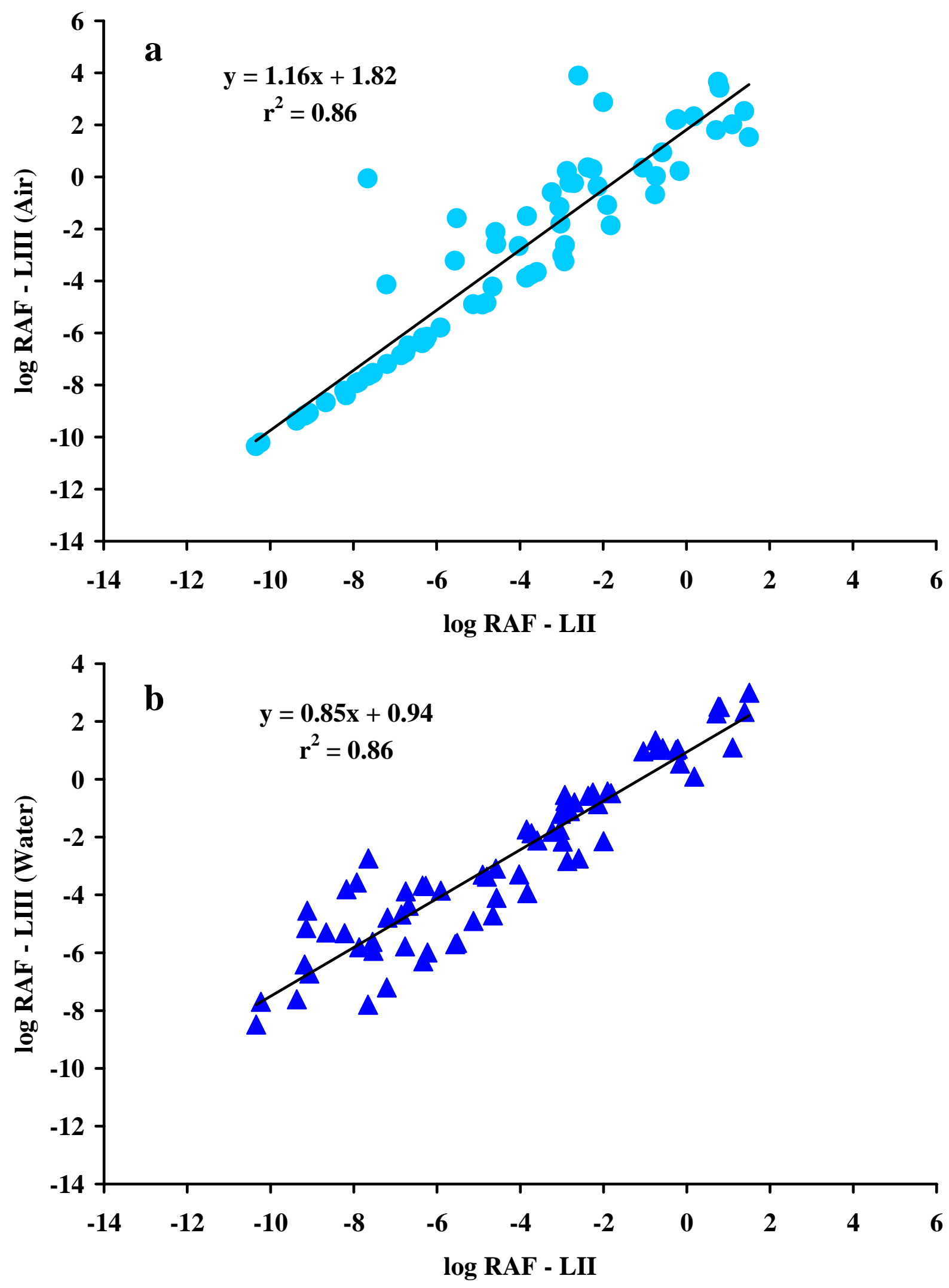

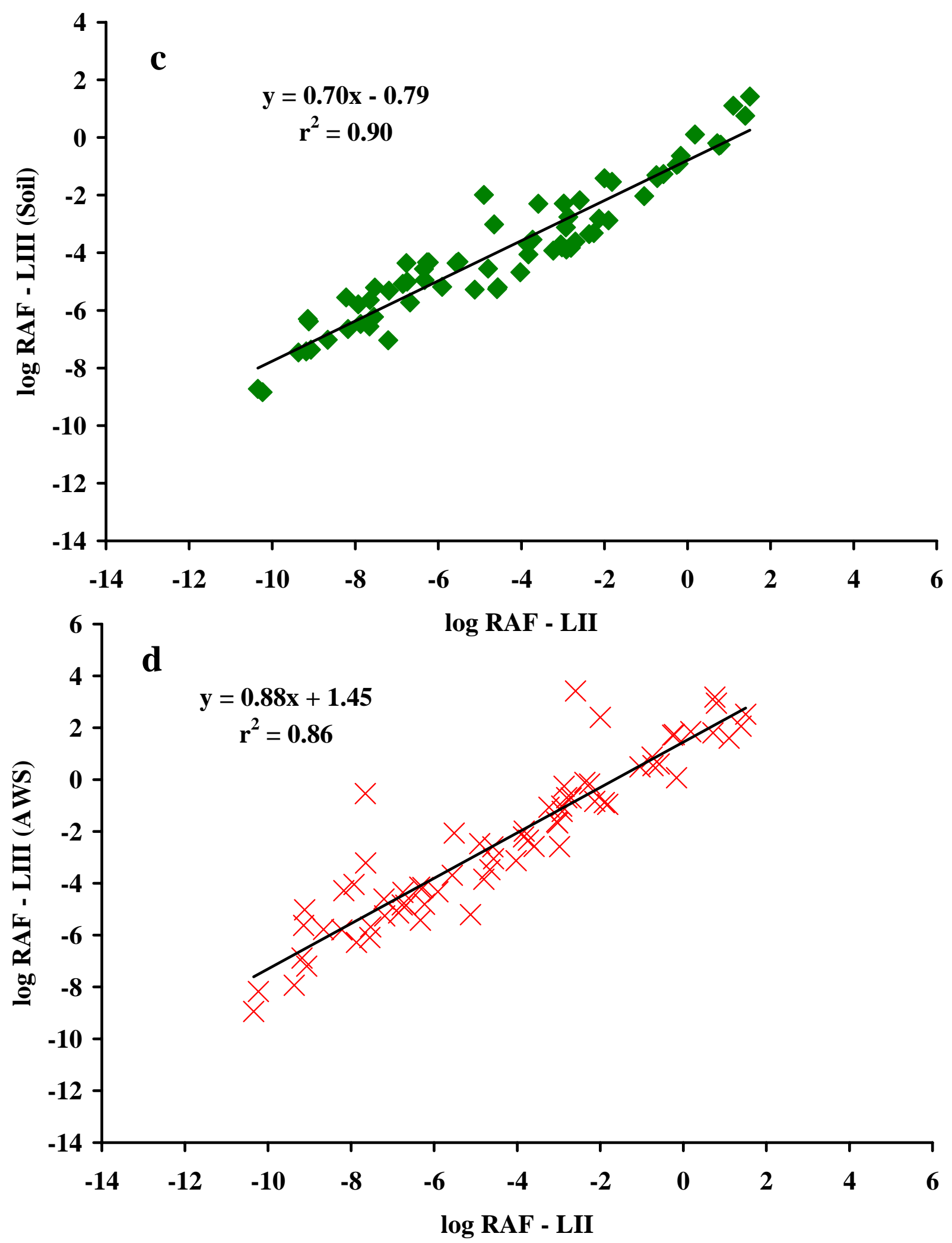

Figure SI-3. RAIDAR risk assessment factor (RAF) comparisons between Level II and Level III mode of entry fate calculations for air (a), water (b), soil (c) and equal unit emission rate to air, water and soil (d), respectively. 
RAIDAR - ES\&T Supporting Information (es0514085)

Table SI-1. Abiotic and biotic media included in the RAIDAR model environment.

\section{Abiotic Media}

Air - bulk

Water - bulk

Soil - bulk

Sediment - bulk

\section{$\underline{\text { Aquatic Organisms }}$}

Plankton (various)

Benthic invertebrate (e.g., shrimp, mussel)

'Pelagic-benthic' fish (e.g., smelt)

'Piscivorous' fish (e.g., salmonid)

Aquatic mammal (e.g., beluga whale)

\section{Vegetation}

Foliage vegetation (e.g., fruit, grass, nuts)

Root vegetation (e.g., vegetables, roots)

\section{Terrestrial Organisms}

Terrestrial invertebrate (e.g., worm)

Terrestrial herbivore (e.g., caribou)

Terrestrial carnivore (e.g., wolf)

Avian omnivore - small (e.g., robin)

Avian omnivore - scavenger (e.g., eagle)

Agricultural - poultry (e.g., chicken)

Agricultural - swine (e.g., pork)

Agricultural - cattle (e.g., beef, bulk dairy)

Human (e.g., adult male)

Table SI - 2. RAIDAR abiotic compartments and compositions for the Level III model calculations. Level II model calculations only consider the solid phase fractions for soil and sediment bulk compartments.

\begin{tabular}{|c|c|c|c|c|c|}
\hline Media & $\begin{array}{c}\text { Area, } \\
\mathbf{m}^{2}\end{array}$ & $\begin{array}{c}\text { Depth, } \\
\text { m }\end{array}$ & $\begin{array}{l}\text { Volume } \\
\text { Fraction }\end{array}$ & $\begin{array}{c}\text { Volume, } \\
\mathbf{m}^{3}\end{array}$ & $\begin{array}{c}\text { Density, } \\
\text { kg/m }\end{array}$ \\
\hline Air (Bulk) & $10^{11}$ & 1000 & & $10^{14}$ & 1.19 \\
\hline Air phase & & & 1 & $10^{14}$ & 1.19 \\
\hline Aerosol phase & & & $2 \times 10^{-11}$ & 2000 & 2400 \\
\hline Water (Bulk) & $10^{10}$ & 20 & & $2 \times 10^{11}$ & 1000 \\
\hline Water phase & & & 1 & $2 \times 10^{11}$ & 1000 \\
\hline Suspended particle phase & & & $5 \times 10^{-6}$ & $10^{6}$ & 1500 \\
\hline Biota phase & & & $10^{-6}$ & $2 \times 10^{5}$ & 1000 \\
\hline Soil (Bulk) & $9 \times 10^{10}$ & 0.2 & & $1.8 \times 10^{10}$ & 1500 \\
\hline Air phase & & & 0.2 & $3.6 \times 10^{9}$ & 1.19 \\
\hline Water phase & & & 0.3 & $5.4 \times 10^{9}$ & 1000 \\
\hline Solid phase & & & 0.5 & $9 \times 10^{9}$ & 2400 \\
\hline Sediment (Bulk) & $10^{10}$ & 0.05 & & $5 \times 10^{8}$ & 1280 \\
\hline Water phase & & & 0.8 & $4 \times 10^{8}$ & 1000 \\
\hline Solid phase & & & 0.2 & $1 \times 10^{8}$ & 2400 \\
\hline
\end{tabular}


Table SI - 3. RAIDAR biotic compartments, compositions and key bioaccumulation parameters. The five rates are calculated using equations and parameters in Table SI-5.

\begin{tabular}{|c|c|c|c|c|c|c|c|c|c|}
\hline Media & $\begin{array}{c}\text { Mass, } \\
\text { kg }\end{array}$ & $\begin{array}{c}\text { Lipid } \\
\text { mass } \\
\text { fraction }\end{array}$ & $\begin{array}{c}\text { Water } \\
\text { mass } \\
\text { fraction }\end{array}$ & $\begin{array}{c}\text { Q, } \\
\text { max } \\
\text { BMF }\end{array}$ & $\begin{array}{c}\text { Respiration } \\
\text { rate, } \\
\mathbf{m}^{3} / \mathbf{h}\end{array}$ & $\begin{array}{c}\text { Drinking } \\
\text { rate, } \\
\mathbf{m}^{3} / \mathbf{h}\end{array}$ & $\begin{array}{c}\text { Feeding } \\
\text { rate, } \\
\mathbf{m}^{3} / \mathbf{h}\end{array}$ & $\begin{array}{c}\text { Growth } \\
\text { rate, } \\
\mathbf{m}^{3} / \mathbf{h}\end{array}$ & $\begin{array}{c}\text { Urination } \\
\text { rate, } \\
\mathbf{m}^{3} / \mathbf{h}\end{array}$ \\
\hline Plankton & NA & 0.01 & 0.79 & NA & NA & NA & NA & NA & NA \\
\hline Benthic invertebrate & NA & 0.05 & 0.75 & NA & NA & NA & NA & NA & NA \\
\hline Pelagic-benthic fish & 0.1 & 0.05 & 0.75 & 4 & 8.7E-04 & NA & $2.1 \mathrm{E}-07$ & 2.1E-09 & NA \\
\hline Piscivorous fish & 2.2 & 0.15 & 0.65 & 8 & $6.5 \mathrm{E}-03$ & NA & $3.0 \mathrm{E}-06$ & $3.0 \mathrm{E}-08$ & NA \\
\hline Aquatic mammal & 1000 & 0.35 & 0.45 & 120 & $8.6 \mathrm{E}+00$ & $2.1 \mathrm{E}-04$ & 8.4E-04 & 8.4E-06 & 2.1E-04 \\
\hline Foliage vegetation & NA & 0.01 & 0.79 & NA & NA & NA & NA & NA & NA \\
\hline Root vegetation & NA & 0.01 & 0.79 & NA & NA & NA & NA & NA & NA \\
\hline Terrestrial invertebrate & NA & 0.02 & 0.78 & NA & NA & NA & NA & NA & NA \\
\hline Terrestrial herbivore & 120 & 0.1 & 0.7 & 4 & $1.6 \mathrm{E}+00$ & $2.8 \mathrm{E}-04$ & $4.2 \mathrm{E}-04$ & $4.2 \mathrm{E}-06$ & $2.8 \mathrm{E}-04$ \\
\hline Terrestrial carnivore & 80 & 0.2 & 0.6 & 120 & $1.1 \mathrm{E}+00$ & 1.9E-04 & $1.1 \mathrm{E}-04$ & $1.1 \mathrm{E}-06$ & $1.9 \mathrm{E}-04$ \\
\hline Avian omnivore - small & 0.25 & 0.05 & 0.75 & 10 & $1.4 \mathrm{E}-02$ & $9.5 \mathrm{E}-07$ & $2.6 \mathrm{E}-06$ & $2.6 \mathrm{E}-08$ & $9.5 \mathrm{E}-07$ \\
\hline Avian scavenger & 4.5 & 0.1 & 0.7 & 60 & 1.3E-01 & 7.2E-06 & 3.0E-05 & 3.0E-07 & 7.2E-06 \\
\hline Poultry & 3 & 0.1 & 0.7 & 6 & $9.7 \mathrm{E}-02$ & $5.4 \mathrm{E}-06$ & $1.1 \mathrm{E}-05$ & $1.1 \mathrm{E}-07$ & $5.4 \mathrm{E}-06$ \\
\hline Swine & 175 & 0.2 & 0.6 & 6 & $2.1 \mathrm{E}+00$ & 8.7E-04 & $1.1 \mathrm{E}-03$ & $1.1 \mathrm{E}-05$ & 8.7E-04 \\
\hline Cattle & 550 & 0.2 & 0.6 & 6 & $5.4 \mathrm{E}+00$ & $2.4 \mathrm{E}-03$ & $2.5 \mathrm{E}-03$ & $2.5 \mathrm{E}-05$ & $2.4 \mathrm{E}-03$ \\
\hline Bulk dairy & NA & 0.08 & 0.72 & NA & NA & NA & NA & NA & NA \\
\hline Eggs & NA & 0.08 & 0.72 & NA & NA & NA & NA & NA & $\mathrm{NA}$ \\
\hline Human & 70 & 0.2 & 0.6 & 100 & $7.5 \mathrm{E}-01$ & $7.6 \mathrm{E}-05$ & $1.0 \mathrm{E}-04$ & $1.0 \mathrm{E}-06$ & $7.6 \mathrm{E}-05$ \\
\hline
\end{tabular}


Table SI - 4. Summary of $\mathrm{Z}$ values $\left(\mathrm{mol} / \mathrm{m}^{3} \cdot \mathrm{Pa}\right)$ and relevant parameters in the model.

\begin{tabular}{lll}
\hline Media & Z value & Parameters and Units \\
\hline Air & $\mathrm{Z}_{\mathrm{A}}=1 / \mathrm{RT}$ & $\mathrm{R}=$ Ideal gas law constant $\left(\mathrm{Pa} \cdot \mathrm{m}^{3} / \mathrm{mol} \cdot \mathrm{K}\right)$ \\
& $\mathrm{Z}_{\mathrm{Q}}=\mathrm{Z}_{\mathrm{A}} \cdot 0.1 \cdot \mathrm{K}_{\mathrm{OA}}$ & Temperature $(\mathrm{K})$ \\
& & $\mathrm{K}_{\mathrm{OA}}=$ octanol-air partition coefficient $=\mathrm{K}_{\mathrm{OW}} / \mathrm{K}_{\mathrm{AW}}$ \\
Aerosol & $\mathrm{K}_{\mathrm{OW}}=$ octanol-water partition coefficient
\end{tabular}


Table SI - 5. Summary of generic bioaccumulation model $D$ value equations, definitions and units. $D$ values have units of $\mathrm{mol} / \mathrm{Pa} \mathbf{h}$, efficiencies, $E_{i}$, are unitless ratios, rates, $G_{i}$, have units of $\mathbf{m}^{3} / \mathbf{h}$.

\begin{tabular}{|c|c|}
\hline Equation & Parameter definition \\
\hline \multirow[t]{4}{*}{$\mathrm{D}_{\mathrm{V}}=\mathrm{E}_{\mathrm{V}} \mathrm{G}_{\mathrm{V}} \mathrm{Z}_{\mathrm{WT}}$} & $\mathrm{D}_{\mathrm{V}}=$ environmental medium exchange rate (i.e., air by respiration or water by ventilation) \\
\hline & $\mathrm{E}_{\mathrm{V}}=$ environmental medium exchange chemical transfer efficiency \\
\hline & $\begin{array}{l}\text { for fish } \mathrm{E}_{\mathrm{V}}=1 /\left(1.85+\left(155 / \mathrm{K}_{\mathrm{OW}}\right)\right) \text { for } \log \mathrm{K}_{\mathrm{OW}} \geq 0.5 \text { and } \mathrm{E}_{\mathrm{V}}=0.02 \text { for } \log \mathrm{K}_{\mathrm{OW}}<0.5 \\
\text { for all other organisms, } \mathrm{E}_{\mathrm{V}}=1\end{array}$ \\
\hline & $\mathrm{G}_{\mathrm{V}}=$ gross flow rate for exchange with the environment (i.e., allometric relationship, see Table SI-5) \\
\hline \multirow[t]{4}{*}{$D_{D}=E_{D} G_{D} Z_{D}$} & $\mathrm{D}_{\mathrm{D}}=$ net chemical uptake from its diet \\
\hline & $\mathrm{E}_{\mathrm{D}}=$ dietary chemical transfer efficiency \\
\hline & $\mathrm{G}_{\mathrm{D}}=$ food ingestion rate \\
\hline & $Z_{D}=$ weighted sum of $Z$ values of all dietary items \\
\hline \multirow[t]{2}{*}{$\mathrm{D}_{\mathrm{W}}=\mathrm{G}_{\mathrm{W}} \mathrm{Z}_{\mathrm{WT}}$} & $\mathrm{D}_{\mathrm{W}}=$ net chemical uptake from water ingestion \\
\hline & $\mathrm{G}_{\mathrm{W}}=$ gross volumetric flow rate for drinking water \\
\hline \multirow[t]{2}{*}{$\mathrm{D}_{\mathrm{E}}=\mathrm{D}_{\mathrm{D}} / \mathrm{Q}$} & $\mathrm{D}_{\mathrm{E}}=$ net chemical fecal egestion \\
\hline & $\mathrm{Q}=$ species-specific theoretical maximum biomagnification factor \\
\hline \multirow[t]{2}{*}{$\mathrm{D}_{\mathrm{G}}=\mathrm{G}_{\mathrm{G}} \mathrm{Z}_{\mathrm{B}}$} & $\mathrm{D}_{\mathrm{G}}=$ growth dilution \\
\hline & $\mathrm{G}_{\mathrm{G}}=$ growth rate \\
\hline \multirow[t]{3}{*}{$\mathrm{D}_{\mathrm{M}}=\mathrm{V}_{\mathrm{B}} \mathrm{Z}_{\mathrm{B}} k_{\mathrm{M}}$} & $\mathrm{D}_{\mathrm{M}}=$ metabolic transformation \\
\hline & $\mathrm{M}_{\mathrm{B}}=$ volume of biota, $\mathrm{m}^{3}$ \\
\hline & $k_{\mathrm{M}}=$ metabolic transformation rate constant, $\mathrm{h}^{-1}$ \\
\hline \multirow[t]{2}{*}{$\mathrm{D}_{\mathrm{U}}=\mathrm{G}_{\mathrm{U}} \mathrm{Z}_{\mathrm{WT}}$} & $\mathrm{D}_{\mathrm{U}}=$ urinary excretion \\
\hline & $\mathrm{G}_{\mathrm{U}}=$ urinary excretion rate \\
\hline
\end{tabular}


Table SI - 6. Equations and parameters describing respiration (i.e., air or water), food ingestion and water intake (i.e., drinking) rates for fish [4], avian and mammalian [7] species in the model. Rates, $G_{i}$, have units of $\mathrm{m}^{3} / \mathrm{h}$, activity factors, $A F_{i}$, are unitless. $M_{B}$ is the ratio of the mass (wet weight, $\mathrm{kg}$ ) of the organism to that of a $1 \mathrm{~kg}$ organism.

Respiration rate $\left(\mathrm{m}^{3} / \mathrm{h}\right)=G_{V}=a \cdot M_{B}{ }^{b} \cdot c /(24 \mathrm{~h} / \mathrm{d})$

\begin{tabular}{|c|c|c|c|c|c|}
\hline Organism class & a, $\mathbf{m}^{3} / \mathbf{d}$ & $\begin{array}{l}\text { b, } \\
\text { unitless }\end{array}$ & $\begin{array}{l}\text { c, } \\
\text { unitless }\end{array}$ & \multicolumn{2}{|c|}{ Details of c } \\
\hline \multirow{3}{*}{ Fish } & \multirow{3}{*}{0.98} & \multirow{3}{*}{0.65} & \multirow{3}{*}{$1 /(\mathrm{DO})$} & \multicolumn{2}{|c|}{$\begin{array}{l}\mathrm{DO} \text {, dissolved oxygen concentration, } \mathrm{mg} \\
\mathrm{O}_{2} / \mathrm{L}=(-0.24 \cdot \mathrm{T}+14.04) \cdot \mathrm{OS}\end{array}$} \\
\hline & & & & \multicolumn{2}{|c|}{$\mathrm{T}=$ mean water temperature $=10^{\circ} \mathrm{C}$} \\
\hline & & & & \multicolumn{2}{|c|}{ OS $=$ oxygen saturation $=0.9$ (unitless) } \\
\hline Avian & 0.40 & 0.77 & $A F_{A}$ & \multicolumn{2}{|c|}{$\mathrm{AF}_{\mathrm{A}}$, avian activity factor $=2.5$} \\
\hline Mammals & 0.55 & 0.80 & $A F_{M}$ & \multicolumn{2}{|c|}{$\mathrm{AF}_{\mathrm{M}}$, mammalian activity factor $=1.5$} \\
\hline Humans & 0.4 & 0.80 & $A F_{H}$ & \multicolumn{2}{|c|}{$\mathrm{AF}_{\mathrm{H}}$, human activity factor $=1.5$} \\
\hline \multicolumn{6}{|c|}{ Food ingestion rate $\left(\mathrm{m}^{3}\right.$ - wet weight $\left./ \mathrm{h}\right), G_{D}=a \cdot M_{B}^{b} \cdot c /\left(24000 \mathrm{~kg} \cdot \mathrm{h} / \mathrm{m}^{3} . d\right)$} \\
\hline \multicolumn{2}{|l|}{ Organism class } & a, kg/d & $\begin{array}{l}\text { b, } \\
\text { unitless }\end{array}$ & $\begin{array}{c}\text { c, } \\
\text { unitless }\end{array}$ & Details of $c$ \\
\hline \multicolumn{2}{|l|}{ Fish } & 0.02 & 0.85 & $e^{0.06 T}$ & $\mathrm{~T}=$ mean water temperature $=10^{\circ} \mathrm{C}$ \\
\hline \multicolumn{2}{|l|}{ Avian - wildlife } & 0.05 & 0.85 & $R_{W D}$ & $\mathrm{R}_{\mathrm{WD}}$, wet / dry ratio $=4$ wet $/$ dry \\
\hline \multicolumn{2}{|c|}{ Avian - agricultural } & 0.05 & 0.85 & $R_{W D}$ & $\mathrm{R}_{\mathrm{WD}}$, wet $/$ dry ratio $=2$ wet $/$ dry \\
\hline \multicolumn{2}{|c|}{ cattle, swine } & 0.16 & 0.72 & \multirow{4}{*}{$R_{W D}$} & \multirow{4}{*}{$\mathrm{R}_{\mathrm{WD}}=4$ wet $/$ dry } \\
\hline \multirow{3}{*}{ Mammals } & & 0.08 & 0.72 & & \\
\hline & & 0.02 & 0.8 & & \\
\hline & mammal & 0.02 & 0.8 & & \\
\hline \multicolumn{2}{|l|}{ Humans } & 0.02 & 0.80 & $R_{W D}$ & $\mathrm{R}_{\mathrm{WD}}=4$ wet $/$ dry \\
\hline
\end{tabular}


Water intake rate $\left(\mathrm{m}^{3} / \mathrm{h}\right), G_{W}=a \cdot M_{B}^{b} \cdot c /\left(24000 \mathrm{~L} . \mathrm{h} / \mathrm{m}^{3} . d\right)$

\begin{tabular}{llccc}
\hline Organism class & a, $\mathbf{L} / \mathbf{d}$ & b, unitless & c, unitless \\
\hline Avian & & 0.06 & 0.7 & 1 \\
& cattle, swine & 0.2 & & \\
\multirow{2}{*}{ Mammals } & terrestrial mammals & 0.09 & 0.90 & 1 \\
& aquatic mammals & 0.01 & & 1 \\
\hline
\end{tabular}

Table SI - 7. Feeding preferences in the representative RAIDAR food webs.

\begin{tabular}{|c|c|c|c|c|c|c|c|c|c|c|c|}
\hline Consumer & & 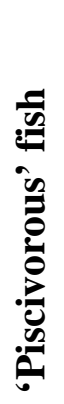 & 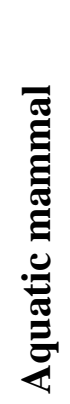 & & 䓪 & 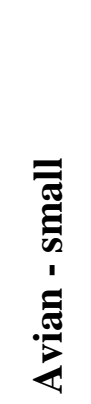 & 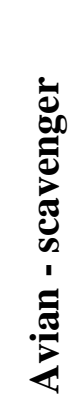 & $\frac{\vec{E}}{\bar{E}}$ & 莡 & نِّتِ & 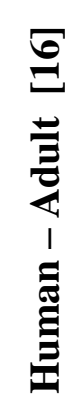 \\
\hline Foliage vegetation & & & & 0.8 & & 0.4 & & 0.9 & 0.8 & 0.8 & 0.22 \\
\hline Root vegetation & & & & 0.2 & & 0.05 & & 0.1 & 0.2 & 0.2 & 0.27 \\
\hline Plankton & 0.5 & & & & & & & & & & \\
\hline Benthic invertebrate & 0.5 & & 0.3 & & & & & & & & 0.01 \\
\hline 'Pelagic-benthic' fish & & 1 & 0.4 & & & & 0.4 & & & & \\
\hline 'Piscivorous' fish & & & 0.3 & & & & 0.15 & & & & 0.02 \\
\hline Aquatic mammal & & & & & & & 0.02 & & & & \\
\hline Terrestrial invertebrate & & & & & & 0.55 & 0.20 & & & & \\
\hline Terrestrial herbivore & & & & & 1 & & 0.18 & & & & \\
\hline Avian - small & & & & & & & 0.05 & & & & \\
\hline Poultry & & & & & & & & & & & 0.04 \\
\hline Pork & & & & & & & & & & & 0.04 \\
\hline Beef & & & & & & & & & & & 0.1 \\
\hline Dairy products & & & & & & & & & & & 0.28 \\
\hline Eggs & & & & & & & & & & & 0.02 \\
\hline
\end{tabular}


Table SI - 8. Illustration of output data for unit emissions $(1 \mathrm{t} / \mathrm{h})$ under different model scenarios for three illustrative chemicals, B(a)P, DCE and HCB. Pov - overall residence time; $L_{A}$ - characteristic travel distance in air; $C_{U}-$ unit emission concentration, $\mathbf{g} / \mathrm{m}^{3}$, wet weight, except Level II soil and sediment are dry weight; BAF = biota, $\mathrm{g} / \mathrm{kg}$ wet weight $/$ surrounding environment, $\mathrm{g} / \mathrm{kg}$

\begin{tabular}{|c|c|c|c|c|c|c|}
\hline $\begin{array}{r}\text { Chemical } \\
\text { Model - MOE }\end{array}$ & $\begin{array}{l}\text { BaP } \\
\text { LII }\end{array}$ & $\begin{array}{c}\text { BaP } \\
\text { LIII-Air }\end{array}$ & $\begin{array}{c}\text { BaP } \\
\text { LIII-Water }\end{array}$ & $\begin{array}{c}\text { BaP } \\
\text { LIII-Soil }\end{array}$ & $\begin{array}{c}\text { BaP } \\
\text { LIII-AWS }\end{array}$ & $\begin{array}{c}\text { BaP } \\
\text { LIII-AWS }\end{array}$ \\
\hline $\mathbf{P}_{\text {ov }}$, days & $8.50 \mathrm{E}+02$ & $5.49 \mathrm{E}+01$ & $2.07 \mathrm{E}+03$ & $8.67 \mathrm{E}+02$ & $8.07 \mathrm{E}+02$ & $8.07 \mathrm{E}+02$ \\
\hline$L_{\mathrm{A}}, \mathbf{k m}$ & $3.24 \mathrm{E}+00$ & $1.01 \mathrm{E}+02$ & N/A & N/A & N/A & N/A \\
\hline Media & $\mathbf{C}_{\mathbf{U}}$ & $\mathrm{C}_{\mathbf{U}}$ & $\mathbf{C}_{\mathbf{U}}$ & $\mathbf{C}_{\mathbf{U}}$ & $\mathbf{C}_{\mathbf{U}}$ & $\log \mathrm{BAF}$ \\
\hline Air - bulk & 2.23E-09 & $6.50 \mathrm{E}-08$ & $1.45 \mathrm{E}-10$ & $7.21 \mathrm{E}-12$ & $2.17 \mathrm{E}-08$ & N/A \\
\hline Water - bulk & $1.19 \mathrm{E}-04$ & $3.17 \mathrm{E}-05$ & $3.88 \mathrm{E}-03$ & $1.02 \mathrm{E}-05$ & $1.31 \mathrm{E}-03$ & N/A \\
\hline Soil - bulk & $2.19 \mathrm{E}+00$ & $5.63 \mathrm{E}-02$ & $1.25 \mathrm{E}-04$ & $1.15 E+00$ & $4.02 \mathrm{E}-01$ & N/A \\
\hline Sediment - bulk & $4.38 \mathrm{E}+00$ & 3.99E-01 & $4.87 \mathrm{E}+01$ & $1.28 \mathrm{E}-01$ & $1.64 \mathrm{E}+01$ & $\mathrm{~N} / \mathrm{A}$ \\
\hline Plankton & $1.99 \mathrm{E}+00$ & $3.26 \mathrm{E}-01$ & $3.99 \mathrm{E}+01$ & $1.05 \mathrm{E}-01$ & $1.34 \mathrm{E}+01$ & 4.01 \\
\hline Benthic invertebrate & $6.68 \mathrm{E}+00$ & $3.04 \mathrm{E}+00$ & $3.72 \mathrm{E}+02$ & $9.80 \mathrm{E}-01$ & $1.25 \mathrm{E}+02$ & 4.98 \\
\hline Benthic-pelagic fish & $1.86 \mathrm{E}+01$ & $5.47 \mathrm{E}+00$ & $6.69 \mathrm{E}+02$ & $1.76 \mathrm{E}+00$ & $2.25 \mathrm{E}+02$ & 5.24 \\
\hline Piscivorous fish & $2.86 \mathrm{E}+02$ & $8.34 \mathrm{E}+01$ & $1.02 \mathrm{E}+04$ & $2.69 \mathrm{E}+01$ & $3.43 \mathrm{E}+03$ & 6.42 \\
\hline Aquatic mammal & $4.59 E+03$ & $1.39 \mathrm{E}+03$ & $1.70 E+05$ & $4.47 \mathrm{E}+02$ & $5.72 E+04$ & 7.64 \\
\hline Foliage vegetation & $1.99 \mathrm{E}+00$ & $5.21 \mathrm{E}+01$ & $1.16 \mathrm{E}-01$ & $5.78 \mathrm{E}-03$ & $1.74 \mathrm{E}+01$ & 5.98 \\
\hline Root vegetation & $1.99 \mathrm{E}+00$ & $1.03 \mathrm{E}-01$ & $2.28 \mathrm{E}-04$ & $2.10 \mathrm{E}+00$ & $7.33 \mathrm{E}-01$ & 0.44 \\
\hline Terrestrial invertebrate & $3.17 \mathrm{E}+00$ & $1.63 \mathrm{E}-01$ & $3.63 \mathrm{E}-04$ & $3.33 \mathrm{E}+00$ & $1.16 \mathrm{E}+00$ & 0.64 \\
\hline Terrestrial herbivore & $3.97 \mathrm{E}+01$ & $8.30 \mathrm{E}+02$ & $1.88 \mathrm{E}+00$ & $8.44 \mathrm{E}+00$ & $2.80 \mathrm{E}+02$ & 7.19 \\
\hline Terrestrial carnivore & $2.68 \mathrm{E}+03$ & $5.60 E+04$ & $1.27 \mathrm{E}+02$ & $5.70 E+02$ & $1.89 \mathrm{E}+04$ & 9.02 \\
\hline Avian small & $5.28 \mathrm{E}+01$ & $5.53 \mathrm{E}+02$ & $1.25 \mathrm{E}+00$ & $3.34 \mathrm{E}+01$ & $1.96 \mathrm{E}+02$ & 7.03 \\
\hline Avian scavenger & $2.67 \mathrm{E}+03$ & $7.50 \mathrm{E}+03$ & $7.87 \mathrm{E}+04$ & $4.55 \mathrm{E}+02$ & $2.89 \mathrm{E}+04$ & 9.20 \\
\hline Poultry & $5.39 \mathrm{E}+01$ & $1.27 \mathrm{E}+03$ & $2.86 \mathrm{E}+00$ & $5.81 \mathrm{E}+00$ & $4.25 \mathrm{E}+02$ & 7.37 \\
\hline Swine & $8.24 \mathrm{E}+01$ & $1.72 \mathrm{E}+03$ & $3.92 \mathrm{E}+00$ & $1.75 \mathrm{E}+01$ & $5.81 \mathrm{E}+02$ & 7.50 \\
\hline Cattle & $8.24 \mathrm{E}+01$ & $1.72 \mathrm{E}+03$ & $3.94 \mathrm{E}+00$ & $1.75 \mathrm{E}+01$ & $5.81 \mathrm{E}+02$ & 7.50 \\
\hline Bulk dairy & $3.46 \mathrm{E}+01$ & $7.24 \mathrm{E}+02$ & $1.65 \mathrm{E}+00$ & $7.36 \mathrm{E}+00$ & $2.44 \mathrm{E}+02$ & 7.13 \\
\hline Egg & $4.38 \mathrm{E}+01$ & $1.03 \mathrm{E}+03$ & $2.32 \mathrm{E}+00$ & $4.72 \mathrm{E}+00$ & $3.46 \mathrm{E}+02$ & 7.28 \\
\hline Human & $1.47 \mathrm{E}+03$ & $2.53 \mathrm{E}+04$ & $5.49 \mathrm{E}+03$ & $3.87 \mathrm{E}+02$ & $1.04 \mathrm{E}+04$ & 8.76 \\
\hline
\end{tabular}


RAIDAR - ES\&T Supporting Information (es0514085)

Table SI- 8. Continued

\begin{tabular}{|c|c|c|c|c|c|c|}
\hline $\begin{array}{r}\text { Chemical } \\
\text { Model - MOE }\end{array}$ & $\begin{array}{c}\text { DCE } \\
\text { LII }\end{array}$ & $\begin{array}{c}\text { DCE } \\
\text { LIII-Air }\end{array}$ & $\begin{array}{c}\text { DCE } \\
\text { LIII-Water }\end{array}$ & $\begin{array}{c}\text { DCE } \\
\text { LIII-Soil }\end{array}$ & $\begin{array}{c}\text { DCE } \\
\text { LIII-AWS }\end{array}$ & $\begin{array}{c}\text { DCE } \\
\text { LIII-AWS }\end{array}$ \\
\hline Pov, days & $5.80 \mathrm{E}+01$ & $5.80 \mathrm{E}+01$ & $5.79 \mathrm{E}+01$ & $8.63 \mathrm{E}+01$ & $6.40 \mathrm{E}+01$ & $6.40 \mathrm{E}+01$ \\
\hline$L_{\mathrm{A}}, \mathbf{k m}$ & $1.92 \mathrm{E}+04$ & $1.94 \mathrm{E}+04$ & N/A & $\mathrm{N} / \mathrm{A}$ & N/A & N/A \\
\hline Media & $\mathbf{C}_{\mathbf{U}}$ & $\mathbf{C}_{\mathbf{U}}$ & $\mathbf{C}_{\mathbf{U}}$ & $\mathbf{C}_{\mathbf{U}}$ & $\mathbf{C}_{\mathbf{U}}$ & $\log$ BAF \\
\hline Air - bulk & 9.30E-07 & 9.31E-07 & 6.88E-07 & 8.96E-07 & 8.38E-07 & $\mathrm{N} / \mathrm{A}$ \\
\hline Water - bulk & $1.90 \mathrm{E}-05$ & $1.43 \mathrm{E}-05$ & $1.79 \mathrm{E}-03$ & $9.77 \mathrm{E}-05$ & $6.34 \mathrm{E}-04$ & $\mathrm{~N} / \mathrm{A}$ \\
\hline Soil - bulk & $9.65 \mathrm{E}-06$ & $1.10 \mathrm{E}-05$ & $8.10 \mathrm{E}-06$ & 5.91E-03 & $1.98 \mathrm{E}-03$ & N/A \\
\hline Sediment - bulk & $1.93 \mathrm{E}-05$ & $1.38 \mathrm{E}-05$ & $1.73 \mathrm{E}-03$ & $9.45 \mathrm{E}-05$ & $6.14 \mathrm{E}-04$ & N/A \\
\hline Plankton & $2.38 \mathrm{E}-05$ & $1.79 \mathrm{E}-05$ & $2.24 \mathrm{E}-03$ & $1.22 \mathrm{E}-04$ & 7.94E-04 & 0.10 \\
\hline Benthic invertebrate & $4.37 \mathrm{E}-05$ & $3.17 \mathrm{E}-05$ & $3.98 \mathrm{E}-03$ & $2.17 \mathrm{E}-04$ & $1.41 \mathrm{E}-03$ & 0.35 \\
\hline Benthic-pelagic fish & 4.38E-05 & $3.28 \mathrm{E}-05$ & $4.12 \mathrm{E}-03$ & $2.25 \mathrm{E}-04$ & $1.46 \mathrm{E}-03$ & 0.36 \\
\hline Piscivorous fish & $1.19 \mathrm{E}-04$ & 8.91E-05 & 1.12E-02 & $6.10 \mathrm{E}-04$ & $3.96 \mathrm{E}-03$ & 0.80 \\
\hline Aquatic mammal & $1.94 E-04$ & $1.94 E-04$ & $2.70 \mathrm{E}-04$ & $1.93 \mathrm{E}-04$ & 2.19E-04 & -0.46 \\
\hline Foliage vegetation & $2.38 \mathrm{E}-05$ & $2.38 \mathrm{E}-05$ & $1.76 \mathrm{E}-05$ & 2.29E-05 & $2.15 \mathrm{E}-05$ & -1.52 \\
\hline Root vegetation & $2.38 \mathrm{E}-05$ & $2.44 \mathrm{E}-05$ & $1.80 \mathrm{E}-05$ & $1.31 \mathrm{E}-02$ & 4.39E-03 & 0.52 \\
\hline Terrestrial invertebrate & $2.88 \mathrm{E}-05$ & $2.94 \mathrm{E}-05$ & $2.18 \mathrm{E}-05$ & $1.59 E-02$ & $5.31 E-03$ & 0.61 \\
\hline Terrestrial herbivore & $6.90 \mathrm{E}-05$ & $6.90 \mathrm{E}-05$ & $7.40 \mathrm{E}-05$ & $1.16 \mathrm{E}-04$ & $8.63 \mathrm{E}-05$ & -0.91 \\
\hline Terrestrial carnivore & $1.19 \mathrm{E}-04$ & 1.19E-04 & $1.27 \mathrm{E}-04$ & $1.17 \mathrm{E}-04$ & $1.21 \mathrm{E}-04$ & -0.77 \\
\hline Avian small & 4.39E-05 & 4.40E-05 & $3.80 \mathrm{E}-05$ & $1.13 \mathrm{E}-04$ & $6.49 \mathrm{E}-05$ & -1.04 \\
\hline Avian scavenger & $6.96 \mathrm{E}-05$ & $6.95 \mathrm{E}-05$ & $1.07 \mathrm{E}-04$ & $1.73 \mathrm{E}-04$ & $1.17 \mathrm{E}-04$ & -0.78 \\
\hline Poultry & $6.88 \mathrm{E}-05$ & $6.88 \mathrm{E}-05$ & $5.82 \mathrm{E}-05$ & 7.66E-05 & $6.79 \mathrm{E}-05$ & -1.02 \\
\hline Swine & $1.20 \mathrm{E}-04$ & $1.19 \mathrm{E}-04$ & $1.79 \mathrm{E}-04$ & $2.81 \mathrm{E}-04$ & $1.93 \mathrm{E}-04$ & -0.56 \\
\hline Cattle & $1.20 \mathrm{E}-04$ & 1.19E-04 & $1.90 \mathrm{E}-04$ & $2.67 \mathrm{E}-04$ & $1.92 \mathrm{E}-04$ & -0.56 \\
\hline Bulk dairy & $5.92 \mathrm{E}-05$ & 5.91E-05 & $9.43 \mathrm{E}-05$ & $1.32 \mathrm{E}-04$ & $9.52 \mathrm{E}-05$ & -0.87 \\
\hline Egg & $5.88 \mathrm{E}-05$ & $5.88 \mathrm{E}-05$ & 4.97E-05 & $6.55 \mathrm{E}-05$ & $5.80 \mathrm{E}-05$ & -1.08 \\
\hline Human & $1.19 \mathrm{E}-04$ & $1.19 \mathrm{E}-04$ & $1.14 \mathrm{E}-04$ & $2.40 \mathrm{E}-04$ & $1.58 \mathrm{E}-04$ & -0.65 \\
\hline
\end{tabular}


RAIDAR - ES\&T Supporting Information (es0514085)

Table SI- 8. Continued

\begin{tabular}{|c|c|c|c|c|c|c|}
\hline $\begin{array}{r}\text { Chemical } \\
\text { Model - MOE } \\
\end{array}$ & $\begin{array}{c}\text { HCB } \\
\text { LII } \\
\end{array}$ & $\begin{array}{c}\text { HCB } \\
\text { LIII-Air } \\
\end{array}$ & $\begin{array}{c}\text { HCB } \\
\text { LIII-Water } \\
\end{array}$ & $\begin{array}{c}\text { HCB } \\
\text { LIII-Soil } \\
\end{array}$ & $\begin{array}{c}\text { HCB } \\
\text { LIII-AWS } \\
\end{array}$ & $\begin{array}{c}\text { HCB } \\
\text { LIII-AWS } \\
\end{array}$ \\
\hline Pov, days & $1.93 \mathrm{E}+03$ & $1.16 \mathrm{E}+03$ & $5.34 \mathrm{E}+03$ & $2.60 \mathrm{E}+03$ & $2.75 \mathrm{E}+03$ & $2.75 \mathrm{E}+03$ \\
\hline$L_{\mathrm{A}}, \mathbf{k m}$ & $6.49 \mathrm{E}+04$ & N/A & N/A & N/A & $\mathrm{N} / \mathrm{A}$ & N/A \\
\hline Media & $\mathbf{C}_{\mathbf{U}}$ & $\mathbf{C}_{\mathbf{U}}$ & $\mathbf{C}_{\mathbf{U}}$ & $\mathbf{C}_{\mathbf{U}}$ & $\mathbf{C}_{\mathbf{U}}$ & $\log \mathbf{B A F}$ \\
\hline Air - bulk & 9.78E-07 & 9.89E-07 & 7.97E-07 & 1.17E-07 & $6.34 \mathrm{E}-07$ & N/A \\
\hline Water - bulk & $1.85 \mathrm{E}-05$ & $1.79 \mathrm{E}-05$ & $2.28 \mathrm{E}-03$ & $2.62 \mathrm{E}-05$ & 7.74E-04 & N/A \\
\hline Soil - bulk & $9.83 \mathrm{E}-02$ & $6.06 \mathrm{E}-03$ & 4.89E-03 & $3.05 \mathrm{E}+00$ & $1.02 \mathrm{E}+00$ & N/A \\
\hline Sediment - bulk & $1.97 \mathrm{E}-01$ & $1.09 \mathrm{E}-01$ & $1.39 E+01$ & $1.60 \mathrm{E}-01$ & 4.73E+00 & $\mathrm{N} / \mathrm{A}$ \\
\hline Plankton & $8.95 \mathrm{E}-02$ & 7.34E-02 & $9.33 \mathrm{E}+00$ & $1.07 \mathrm{E}-01$ & $3.17 \mathrm{E}+00$ & 3.61 \\
\hline Benthic invertebrate & $3.00 \mathrm{E}-01$ & $8.35 \mathrm{E}-01$ & $1.06 \mathrm{E}+02$ & $1.22 \mathrm{E}+00$ & $3.61 \mathrm{E}+01$ & 4.67 \\
\hline Benthic-pelagic fish & $6.09 \mathrm{E}-01$ & $9.21 \mathrm{E}-01$ & $1.17 \mathrm{E}+02$ & $1.35 \mathrm{E}+00$ & $3.98 \mathrm{E}+01$ & 4.71 \\
\hline Piscivorous fish & $6.89 \mathrm{E}+00$ & $1.01 \mathrm{E}+01$ & $1.28 \mathrm{E}+03$ & $1.48 \mathrm{E}+01$ & $4.36 \mathrm{E}+02$ & 5.75 \\
\hline Aquatic mammal & $8.78 E+01$ & $1.41 E+02$ & $1.79 E+04$ & $2.05 \mathrm{E}+02$ & $6.07 E+03$ & 6.89 \\
\hline Foliage vegetation & $8.95 \mathrm{E}-02$ & $9.06 \mathrm{E}-02$ & 7.30E-02 & $1.07 \mathrm{E}-02$ & $5.81 \mathrm{E}-02$ & 2.04 \\
\hline Root vegetation & 8.95E-02 & $1.10 \mathrm{E}-02$ & 8.90E-03 & $5.55 \mathrm{E}+00$ & $1.86 \mathrm{E}+00$ & 0.44 \\
\hline Terrestrial invertebrate & $1.42 \mathrm{E}-01$ & $1.75 \mathrm{E}-02$ & $1.41 \mathrm{E}-02$ & $8.81 \mathrm{E}+00$ & $2.95 \mathrm{E}+00$ & 0.64 \\
\hline Terrestrial herbivore & $1.64 \mathrm{E}+00$ & $1.38 \mathrm{E}+00$ & $1.13 \mathrm{E}+00$ & $1.96 \mathrm{E}+01$ & $7.36 \mathrm{E}+00$ & 4.14 \\
\hline Terrestrial carnivore & $6.70 \mathrm{E}+01$ & $5.65 \mathrm{E}+01$ & $4.66 \mathrm{E}+01$ & $7.98 E+02$ & $3.00 \mathrm{E}+02$ & 5.75 \\
\hline Avian small & $1.80 \mathrm{E}+00$ & $9.05 \mathrm{E}-01$ & $7.40 \mathrm{E}-01$ & $6.39 \mathrm{E}+01$ & $2.18 \mathrm{E}+01$ & 4.61 \\
\hline Avian scavenger & $5.36 \mathrm{E}+01$ & $6.83 \mathrm{E}+01$ & $7.53 \mathrm{E}+03$ & $5.08 \mathrm{E}+02$ & $2.70 \mathrm{E}+03$ & 6.70 \\
\hline Poultry & $1.84 \mathrm{E}+00$ & $1.72 \mathrm{E}+00$ & $1.40 \mathrm{E}+00$ & $1.05 \mathrm{E}+01$ & $4.55 \mathrm{E}+00$ & 3.93 \\
\hline Swine & $3.52 \mathrm{E}+00$ & $2.95 \mathrm{E}+00$ & $2.43 \mathrm{E}+00$ & $4.30 \mathrm{E}+01$ & $1.61 \mathrm{E}+01$ & 4.48 \\
\hline Cattle & $3.50 \mathrm{E}+00$ & $2.93 \mathrm{E}+00$ & $2.44 \mathrm{E}+00$ & $4.27 \mathrm{E}+01$ & $1.60 \mathrm{E}+01$ & 4.48 \\
\hline Bulk dairy & $1.47 \mathrm{E}+00$ & $1.23 \mathrm{E}+00$ & $1.02 \mathrm{E}+00$ & $1.79 \mathrm{E}+01$ & $6.73 \mathrm{E}+00$ & 4.10 \\
\hline Egg & $1.50 \mathrm{E}+00$ & $1.40 \mathrm{E}+00$ & $1.14 \mathrm{E}+00$ & $8.57 \mathrm{E}+00$ & $3.70 \mathrm{E}+00$ & 3.84 \\
\hline Human & $3.97 \mathrm{E}+01$ & $3.26 \mathrm{E}+01$ & $5.14 \mathrm{E}+02$ & $6.27 \mathrm{E}+02$ & $3.91 \mathrm{E}+02$ & 5.87 \\
\hline
\end{tabular}


Table SI - 9. Physical-chemical property [15,17] and selected half-life data used as RAIDAR input for 70 chemicals.

\begin{tabular}{|c|c|c|c|c|c|c|c|c|c|c|c|c|}
\hline $\begin{array}{c}\text { Chemical } \\
\text { ID \# }\end{array}$ & CAS \# & $\begin{array}{l}\text { Molar } \\
\text { Mass, } \\
\text { g/mol }\end{array}$ & $\begin{array}{c}\text { Water } \\
\text { Solubility, } \\
\mathbf{g} / \mathbf{m}^{3}\end{array}$ & $\begin{array}{c}\text { Vapor } \\
\text { Pressure, } \\
\text { Pa }\end{array}$ & $\begin{array}{c}\log \\
\text { Kow }_{\text {OW }}\end{array}$ & pKa & $\begin{array}{c}\mathrm{L}(\mathrm{E}) \mathrm{C}_{50} \\
\mathrm{mg} / \mathrm{L}\end{array}$ & $\begin{array}{c}\log \\
\text { BCF }^{\mathbf{a}} \\
\mathbf{L} / \mathbf{k g}\end{array}$ & $\begin{array}{l}t_{1 / 2} \text { in } \\
\text { Air, } \\
\text { days }\end{array}$ & $\begin{array}{c}\mathbf{t}_{1 / 2} \text { in } \\
\text { Water, } \\
\text { days }\end{array}$ & $\begin{array}{l}\mathrm{t}_{1 / 2} \text { in } \\
\text { Soil, } \\
\text { days }\end{array}$ & $\begin{array}{c}\mathbf{t}_{1 / 2} \text { in } \\
\text { Sediment, } \\
\text { days }\end{array}$ \\
\hline 1 & $50-00-0$ & 30.03 & $5.70 \mathrm{E}+04$ & $1.00 \mathrm{E}+05$ & 0.35 & NA & $3.0 \mathrm{E}-01$ & 0.03 & 1.31 & 5 & 10 & 30 \\
\hline 2 & $50-32-8$ & 252.32 & $3.80 \mathrm{E}-03$ & $7.00 \mathrm{E}-07$ & 6.04 & NA & $5.0 \mathrm{E}-03$ & 2.69 & 0.21 & 200 & 600 & 1800 \\
\hline 3 & $51-28-5$ & 184.11 & $1.97 \mathrm{E}+03$ & $1.72 \mathrm{E}-03$ & 1.67 & 4.0 & $3.9 \mathrm{E}-01$ & 0.57 & 15.93 & 80 & 160 & 10 \\
\hline 4 & $67-56-1$ & 32.04 & $1.00 \mathrm{E}+06$ & $1.69 \mathrm{E}+04$ & -0.77 & 15.2 & $3.3 \mathrm{E}+03$ & -0.02 & 17.06 & 7 & 14 & 20 \\
\hline 5 & $67-63-0$ & 60.10 & $4.02 \mathrm{E}+05$ & $6.05 \mathrm{E}+03$ & 0.05 & 15.3 & $6.6 \mathrm{E}+03$ & 0.00 & 1.47 & 8 & 16 & 20 \\
\hline 6 & $67-66-3$ & 119.38 & $2.10 \mathrm{E}+03$ & $2.62 E+04$ & 1.97 & NA & $3.5 \mathrm{E}+01$ & 0.78 & 91.31 & 45 & 75 & 50 \\
\hline 7 & $67-72-1$ & 236.74 & $8.15 \mathrm{E}+00$ & $2.79 \mathrm{E}+01$ & 4.14 & NA & $8.6 \mathrm{E}-01$ & 2.84 & 499.50 & 280 & 400 & 600 \\
\hline 8 & $68-12-2$ & 73.10 & $9.78 \mathrm{E}+05$ & $5.15 \mathrm{E}+02$ & -1.01 & -0.4 & $1.3 \mathrm{E}+04$ & -0.20 & 0.61 & 10 & 20 & 60 \\
\hline 9 & $70-30-4$ & 406.91 & $3.84 \mathrm{E}-03$ & $1.11 \mathrm{E}-08$ & 7.54 & 7.3 & $2.1 \mathrm{E}-02$ & 2.06 & 4.89 & 200 & 400 & 1200 \\
\hline 10 & $71-36-3$ & 74.12 & $7.67 \mathrm{E}+04$ & $1.04 \mathrm{E}+03$ & 0.88 & 15.2 & $1.2 \mathrm{E}+03$ & 0.13 & 1.55 & 5 & 10 & 20 \\
\hline 11 & $71-43-2$ & 78.11 & $2.00 \mathrm{E}+03$ & $1.27 \mathrm{E}+04$ & 2.13 & NA & $9.2 \mathrm{E}+00$ & 0.88 & 5.46 & 25 & 50 & 200 \\
\hline 12 & $74-85-1$ & 28.05 & $3.45 \mathrm{E}+03$ & $1.00 \mathrm{E}+05$ & 1.13 & NA & $1.0 \mathrm{E}+02$ & 0.21 & 1.05 & 11 & 22 & 66 \\
\hline 13 & $76-60-8$ & 698.02 & $2.66 \mathrm{E}-05$ & $1.00 \mathrm{E}-09$ & 7.86 & 6.7 & 7.0E-03 & 4.11 & 2.46 & 200 & 400 & 1200 \\
\hline 14 & $77-54-3$ & 264.41 & $5.50 \mathrm{E}-01$ & $6.86 \mathrm{E}-02$ & 5.33 & NA & $1.2 \mathrm{E}-01$ & 3.98 & 0.90 & 50 & 100 & 300 \\
\hline 15 & $78-87-5$ & 112.99 & $2.17 \mathrm{E}+03$ & $6.05 \mathrm{E}+03$ & 1.98 & NA & $8.3 \mathrm{E}+01$ & 0.30 & 23.62 & 60 & 120 & 360 \\
\hline 16 & $78-93-3$ & 72.11 & $7.61 \mathrm{E}+04$ & $1.27 \mathrm{E}+04$ & 0.29 & NA & $4.0 \mathrm{E}+02$ & 0.02 & 7.96 & 12 & 24 & 72 \\
\hline 17 & 79-01-6 & 131.39 & $7.79 \mathrm{E}+02$ & $9.18 \mathrm{E}+03$ & 2.42 & NA & $3.1 \mathrm{E}+00$ & 1.23 & 13.21 & 60 & 120 & 300 \\
\hline 18 & $79-34-5$ & 167.85 & $5.73 E+02$ & $6.14 \mathrm{E}+02$ & 2.39 & NA & $1.2 \mathrm{E}+01$ & 0.91 & 50.36 & 35 & 70 & 50 \\
\hline 19 & $79-92-5$ & 136.24 & $6.28 \mathrm{E}+00$ & $2.37 \mathrm{E}+02$ & 4.22 & NA & $1.8 \mathrm{E}+00$ & 2.94 & 0.16 & 25 & 50 & 150 \\
\hline 20 & 79-94-7 & 543.88 & $1.00 \mathrm{E}-03$ & $4.61 \mathrm{E}-09$ & 7.20 & 7.1 & $6.0 \mathrm{E}-02$ & 3.34 & 3.60 & 100 & 200 & 400 \\
\hline 21 & $84-74-2$ & 278.35 & $1.12 \mathrm{E}+01$ & $2.68 \mathrm{E}-03$ & 4.50 & NA & $4.0 \mathrm{E}-01$ & 3.13 & 1.15 & 4 & 12 & 36 \\
\hline
\end{tabular}




\begin{tabular}{|c|c|c|c|c|c|c|c|c|c|c|c|c|}
\hline $\begin{array}{c}\text { Chemical } \\
\text { ID \# }\end{array}$ & CAS \# & $\begin{array}{l}\text { Molar } \\
\text { Mass, } \\
\text { g/mol }\end{array}$ & $\begin{array}{c}\text { Water } \\
\text { Solubility, } \\
\mathbf{g} / \mathbf{m}^{3}\end{array}$ & $\begin{array}{c}\text { Vapor } \\
\text { Pressure, } \\
\text { Pa }\end{array}$ & $\begin{array}{c}\log \\
\text { Kow }_{\text {OW }}\end{array}$ & pKa & $\begin{array}{c}\mathrm{L}(\mathrm{E}) \mathrm{C}_{50} \\
\mathrm{mg} / \mathrm{L}\end{array}$ & $\begin{array}{c}\log \\
\mathbf{B C F}^{\mathbf{a}} \\
\mathbf{L} / \mathbf{k g}\end{array}$ & $\begin{array}{l}t_{1 / 2} \text { in } \\
\text { Air, } \\
\text { days }\end{array}$ & $\begin{array}{c}\mathbf{t}_{1 / 2} \text { in } \\
\text { Water, } \\
\text { days }\end{array}$ & $\begin{array}{l}t_{1 / 2} \text { in } \\
\text { Soil, } \\
\text { days }\end{array}$ & $\begin{array}{c}\mathbf{t}_{1 / 2} \text { in } \\
\text { Sediment } \\
\quad \text { days }\end{array}$ \\
\hline 22 & $85-01-8$ & 178.24 & $6.77 \mathrm{E}-01$ & $1.49 \mathrm{E}-02$ & 4.46 & NA & $5.1 \mathrm{E}-02$ & 3.16 & 0.82 & 50 & 150 & 450 \\
\hline 23 & $85-68-7$ & 312.37 & $9.49 \mathrm{E}-01$ & $1.10 \mathrm{E}-03$ & 4.73 & NA & $6.8 \mathrm{E}-01$ & 3.42 & 0.97 & 5 & 15 & 45 \\
\hline 24 & $87-86-5$ & 266.34 & $3.09 \mathrm{E}+00$ & $1.46 \mathrm{E}-02$ & 5.12 & 4.7 & $9.0 \mathrm{E}-02$ & 1.68 & 19.06 & 220 & 440 & 1200 \\
\hline 25 & $91-20-3$ & 128.18 & $1.42 \mathrm{E}+02$ & $5.39 \mathrm{E}+00$ & 3.30 & NA & 5.1E-01 & 2.01 & 0.49 & 25 & 75 & 225 \\
\hline 26 & $95-50-1$ & 147.00 & $9.20 \mathrm{E}+01$ & $1.81 \mathrm{E}+02$ & 3.43 & NA & 7.4E-01 & 2.14 & 26.01 & 50 & 100 & 350 \\
\hline 27 & $95-63-6$ & 120.20 & $7.96 \mathrm{E}+01$ & $2.16 \mathrm{E}+02$ & 3.63 & NA & $3.6 \mathrm{E}+00$ & 1.90 & 0.64 & 20 & 40 & 120 \\
\hline 28 & 101-61-1 & 254.38 & $4.14 \mathrm{E}+00$ & $4.43 \mathrm{E}-04$ & 4.37 & 6.0 & $9.3 \mathrm{E}-02$ & 3.44 & 0.05 & 150 & 300 & 900 \\
\hline 29 & $104-40-5$ & 220.36 & $1.57 \mathrm{E}+00$ & $9.14 \mathrm{E}-02$ & 5.76 & 10.4 & $4.5 \mathrm{E}-02$ & 2.43 & 0.21 & 15 & 30 & 90 \\
\hline 30 & $106-46-7$ & 147.00 & $9.02 \mathrm{E}+01$ & $1.33 \mathrm{E}+02$ & 3.44 & NA & $7.0 \mathrm{E}-01$ & 2.15 & 26.01 & 50 & 100 & 350 \\
\hline 31 & $107-06-2$ & 98.96 & $8.61 \mathrm{E}+03$ & $1.05 \mathrm{E}+04$ & 1.48 & NA & $1.2 \mathrm{E}+02$ & 0.30 & 40.18 & 40 & 120 & 360 \\
\hline 32 & $107-21-1$ & 62.07 & $1.00 \mathrm{E}+06$ & $1.23 E+01$ & -1.36 & 15.9 & $7.8 \mathrm{E}+03$ & -0.02 & 1.29 & 4 & 8 & 24 \\
\hline 33 & $108-10-1$ & 100.16 & $8.89 \mathrm{E}+03$ & $2.65 E+03$ & 1.31 & NA & $4.6 \mathrm{E}+02$ & 0.30 & 1.21 & 15 & 30 & 90 \\
\hline 34 & $108-88-3$ & 92.14 & $5.73 \mathrm{E}+02$ & $3.79 E+03$ & 2.73 & NA & $3.0 \mathrm{E}+00$ & 1.45 & 2.04 & 14 & 28 & 84 \\
\hline 35 & $108-90-7$ & 112.56 & $4.01 \mathrm{E}+02$ & $1.60 \mathrm{E}+03$ & 2.84 & NA & $1.7 \mathrm{E}+01$ & 1.56 & 7.74 & 35 & 70 & 210 \\
\hline 36 & $110-54-3$ & 86.18 & $9.50 \mathrm{E}+00$ & $2.02 \mathrm{E}+04$ & 3.90 & NA & $3.5 \mathrm{E}+00$ & 2.61 & 1.96 & 6 & 12 & 36 \\
\hline 37 & $110-82-7$ & 84.16 & $4.30 \mathrm{E}+01$ & $1.29 \mathrm{E}+04$ & 3.44 & NA & $2.4 \mathrm{E}+00$ & 1.85 & 1.26 & 15 & 30 & 90 \\
\hline 38 & $111-42-2$ & 105.14 & $1.00 \mathrm{E}+06$ & $3.73 \mathrm{E}-02$ & -1.43 & 15.1 & $2.9 \mathrm{E}+01$ & -0.02 & 0.12 & 4 & 8 & 24 \\
\hline 39 & $115-07-1$ & 42.08 & $1.16 \mathrm{E}+03$ & $1.00 \mathrm{E}+05$ & 1.77 & NA & $6.5 \mathrm{E}+01$ & 0.60 & 0.28 & 12 & 24 & 72 \\
\hline 40 & $117-81-7$ & 390.57 & $1.13 \mathrm{E}-03$ & $1.30 \mathrm{E}-03$ & 7.60 & NA & $6.9 \mathrm{E}-01$ & 3.13 & 0.49 & 10 & 30 & 90 \\
\hline 41 & 118-74-1 & 284.78 & $5.00 \mathrm{E}-03$ & $2.30 \mathrm{E}-03$ & 5.50 & NA & $3.0 \mathrm{E}-02$ & 4.11 & 387.52 & 900 & 1800 & 5400 \\
\hline 42 & $120-12-7$ & 178.24 & $6.91 \mathrm{E}-01$ & $3.56 \mathrm{E}-04$ & 4.45 & NA & $1.3 \mathrm{E}-03$ & 2.81 & 0.27 & 50 & 150 & 450 \\
\hline 43 & $120-83-2$ & 163.00 & $6.14 \mathrm{E}+02$ & $8.76 E+00$ & 3.06 & 8.1 & $2.6 \mathrm{E}-01$ & 1.40 & 3.58 & 40 & 80 & 240 \\
\hline 44 & $124-18-5$ & 142.29 & $1.25 \mathrm{E}+00$ & $2.31 \mathrm{E}+02$ & 5.01 & NA & $2.8 \mathrm{E}-02$ & 3.69 & 0.96 & 6 & 12 & 36 \\
\hline
\end{tabular}




\begin{tabular}{|c|c|c|c|c|c|c|c|c|c|c|c|c|}
\hline $\begin{array}{c}\text { Chemical } \\
\text { ID \# }\end{array}$ & CAS \# & $\begin{array}{l}\text { Molar } \\
\text { Mass, } \\
\text { g/mol }\end{array}$ & $\begin{array}{c}\text { Water } \\
\text { Solubility, } \\
\mathbf{g} / \mathbf{m}^{3}\end{array}$ & $\begin{array}{c}\text { Vapor } \\
\text { Pressure, } \\
\text { Pa }\end{array}$ & $\begin{array}{c}\log \\
\text { Kow }_{\text {OW }}\end{array}$ & pKa & $\begin{array}{c}\mathrm{L}(\mathrm{E}) \mathrm{C}_{50} \\
\mathrm{mg} / \mathrm{L}\end{array}$ & $\begin{array}{c}\log \\
\mathbf{B C F}^{\mathbf{a}} \\
\mathbf{L} / \mathbf{k g}\end{array}$ & $\begin{array}{l}t_{1 / 2} \text { in } \\
\text { Air, } \\
\text { days }\end{array}$ & $\begin{array}{c}\mathbf{t}_{1 / 2} \text { in } \\
\text { Water, } \\
\text { days }\end{array}$ & $\begin{array}{l}t_{1 / 2} \text { in } \\
\text { Soil, } \\
\text { days }\end{array}$ & $\begin{array}{c}\mathbf{t}_{1 / 2} \text { in } \\
\text { Sediment } \\
\quad \text { days }\end{array}$ \\
\hline 45 & $127-18-4$ & 165.83 & $8.03 \mathrm{E}+01$ & $2.47 \mathrm{E}+03$ & 3.40 & NA & $1.1 \mathrm{E}+01$ & 1.79 & 49.84 & 90 & 200 & 600 \\
\hline 46 & $129-00-0$ & 202.26 & $2.25 \mathrm{E}-01$ & $3.27 \mathrm{E}-04$ & 4.88 & NA & $2.3 \mathrm{E}-04$ & 2.99 & 0.21 & 200 & 600 & 1800 \\
\hline 47 & $603-35-0$ & 262.29 & $2.79 \mathrm{E}-01$ & $1.36 \mathrm{E}-03$ & 5.69 & NA & $9.1 \mathrm{E}-01$ & 4.26 & 1.83 & 30 & 60 & 180 \\
\hline 48 & $608-93-5$ & 250.34 & $9.06 \mathrm{E}-01$ & $2.92 \mathrm{E}-01$ & 5.17 & NA & 5.9E-01 & 3.83 & 156.02 & 280 & 560 & 1680 \\
\hline 49 & $1163-19-5$ & 959.17 & $1.00 \mathrm{E}-05$ & $1.00 \mathrm{E}-09$ & 9.00 & NA & $4.4 \mathrm{E}+00$ & 3.00 & 240.93 & 550 & 1000 & 3000 \\
\hline 50 & $1222-05-5$ & 258.41 & $9.57 \mathrm{E}-02$ & $1.17 \mathrm{E}-02$ & 6.26 & NA & $6.5 \mathrm{E}-01$ & 4.56 & 0.28 & 100 & 300 & 900 \\
\hline 51 & $1330-78-5$ & 368.37 & $1.84 \mathrm{E}-02$ & $8.00 \mathrm{E}-05$ & 6.34 & 9.4 & $2.2 \mathrm{E}+00$ & 2.90 & 0.78 & 25 & 50 & 150 \\
\hline 52 & $1582-09-8$ & 335.29 & $2.09 \mathrm{E}-01$ & $6.47 \mathrm{E}-03$ & 5.34 & NA & $5.0 \mathrm{E}-03$ & 3.64 & 0.45 & 120 & 200 & 40 \\
\hline 53 & $1897-45-6$ & 265.91 & $2.60 \mathrm{E}+01$ & $4.53 \mathrm{E}-05$ & 3.05 & NA & $1.1 \mathrm{E}-02$ & 1.40 & 633.43 & 70 & 140 & 420 \\
\hline 54 & $1912-24-9$ & 215.69 & $2.14 \mathrm{E}+02$ & $4.00 \mathrm{E}-05$ & 2.61 & 2.4 & $1.1 \mathrm{E}-02$ & 0.90 & 0.39 & 125 & 250 & 750 \\
\hline 55 & $3380-34-5$ & 289.55 & $4.62 \mathrm{E}+00$ & $6.20 \mathrm{E}-04$ & 4.76 & 7.8 & $2.8 \mathrm{E}-03$ & 1.57 & 0.66 & 85 & 170 & 510 \\
\hline 56 & $3846-71-7$ & 323.44 & $1.50 \mathrm{E}-01$ & $1.47 \mathrm{E}-07$ & 6.27 & 9.4 & $2.7 \mathrm{E}-02$ & 4.57 & 0.79 & 105 & 210 & 630 \\
\hline 57 & $4979-32-2$ & 346.55 & $5.38 \mathrm{E}-02$ & $6.05 \mathrm{E}-06$ & 5.95 & 4.1 & $5.5 \mathrm{E}-02$ & 4.43 & 0.09 & 40 & 80 & 240 \\
\hline 58 & $5285-60-9$ & 310.49 & $6.90 \mathrm{E}-02$ & $5.45 \mathrm{E}-05$ & 6.08 & 6.6 & $6.2 \mathrm{E}-03$ & 4.50 & 0.07 & 90 & 180 & 540 \\
\hline 59 & $6386-38-5$ & 292.42 & $2.46 \mathrm{E}+00$ & $1.80 \mathrm{E}-04$ & 5.06 & 12.7 & $2.5 \mathrm{E}-01$ & 3.73 & 0.56 & 40 & 80 & 240 \\
\hline 60 & $13560-89-9$ & 653.73 & $1.00 \mathrm{E}-05$ & $9.41 \mathrm{E}-08$ & 9.00 & NA & PJ & 2.58 & 0.47 & 800 & 1600 & 4800 \\
\hline 61 & $15646-96-5$ & 210.28 & $5.73 \mathrm{E}+00$ & $8.07 \mathrm{E}-01$ & 4.48 & NA & $1.4 \mathrm{E}+00$ & 3.18 & 1.25 & 10 & 30 & 90 \\
\hline 62 & $23593-75-1$ & 344.85 & $2.98 \mathrm{E}-02$ & $2.84 \mathrm{E}-07$ & 6.26 & 6.1 & $1.9 \mathrm{E}-02$ & 4.56 & 0.23 & 135 & 270 & 810 \\
\hline 63 & $25973-55-1$ & 351.50 & $1.48 \mathrm{E}-02$ & $2.57 \mathrm{E}-08$ & 7.25 & 8.7 & $1.3 \mathrm{E}-02$ & 4.42 & 0.68 & 125 & 250 & 750 \\
\hline 64 & $26140-60-3$ & 230.31 & $2.15 \mathrm{E}-01$ & $4.56 \mathrm{E}-05$ & 6.03 & NA & $1.6 \mathrm{E}-01$ & 4.47 & 1.16 & 40 & 80 & 240 \\
\hline 65 & $32534-81-9$ & 564.69 & $7.86 \mathrm{E}-05$ & $3.25 \mathrm{E}-06$ & 7.66 & NA & PJ & 3.87 & 19.07 & 500 & 1000 & 3000 \\
\hline 66 & $32536-52-0$ & 801.38 & $1.00 \mathrm{E}-05$ & 4.91E-09 & 9.00 & NA & $\mathrm{PJ}$ & 2.58 & 85.60 & 800 & 1600 & 4800 \\
\hline 67 & $54079-53-7$ & 413.57 & $2.54 \mathrm{E}-04$ & $1.00 \mathrm{E}-09$ & 7.88 & 1.6 & $4.0 \mathrm{E}-03$ & 4.10 & 0.04 & 75 & 150 & 450 \\
\hline
\end{tabular}




\begin{tabular}{|c|c|c|c|c|c|c|c|c|c|c|c|c|}
\hline $\begin{array}{c}\text { Chemical } \\
\text { ID \# }\end{array}$ & CAS \# & $\begin{array}{l}\text { Molar } \\
\text { Mass, } \\
\text { g/mol }\end{array}$ & $\begin{array}{c}\text { Water } \\
\text { Solubility, } \\
\mathbf{g} / \mathbf{m}^{3}\end{array}$ & $\begin{array}{c}\text { Vapor } \\
\text { Pressure, } \\
\text { Pa }\end{array}$ & $\begin{array}{c}\log \\
K_{\text {OW }}\end{array}$ & pKa & $\begin{array}{c}\mathrm{L}(\mathrm{E}) \mathrm{C}_{50} \\
\mathrm{mg} / \mathrm{L}\end{array}$ & $\begin{array}{c}\log \\
\mathbf{B C F} \\
\mathbf{L} / \mathbf{k g}\end{array}$ & $\begin{array}{l}t_{1 / 2} \text { in } \\
\text { Air, } \\
\text { days }\end{array}$ & $\begin{array}{c}\mathbf{t}_{1 / 2} \text { in } \\
\text { Water, } \\
\text { days }\end{array}$ & $\begin{array}{l}t_{1 / 2} \text { in } \\
\text { Soil, } \\
\text { days }\end{array}$ & $\begin{array}{c}\mathbf{t}_{1 / 2} \text { in } \\
\text { Sediment, } \\
\text { days }\end{array}$ \\
\hline 68 & $54827-17-7$ & 240.35 & $8.23 \mathrm{E}+00$ & $6.69 \mathrm{E}-06$ & 4.11 & 4.5 & $2.7 \mathrm{E}+00$ & 2.81 & 0.14 & 90 & 180 & 540 \\
\hline 69 & $63936-56-1$ & 880.28 & $1.00 \mathrm{E}-05$ & $1.00 \mathrm{E}-09$ & 9.00 & NA & PJ & 2.58 & 138.65 & 800 & 1600 & 4800 \\
\hline 70 & 89347-09-1 & 466.84 & $2.30 \mathrm{E}-05$ & $1.18 \mathrm{E}-09$ & 9.00 & NA & PJ & 3.31 & 0.02 & 200 & 400 & 1200 \\
\hline
\end{tabular}

${ }^{\mathbf{a}} \mathrm{BCF}$ value used to estimate internal effect concentration and toxic ratio

$\mathrm{PJ}$ - denoted by Environment Canada as professional judgment; selected as a narcotic (i.e., internal effect concentration $=5 \mathrm{mmol} / \mathrm{kg}$ ) 
Table SI - 10. Summary RAIDAR Level II model output for 70 chemicals.

\begin{tabular}{|c|c|c|c|c|c|c|c|c|}
\hline ID \# & CAS \# & $\begin{array}{c}\mathbf{E}_{\mathrm{A}} \\
\text { t / year }\end{array}$ & $\begin{array}{c}L \text { II E }_{C} \\
\text { t / year }\end{array}$ & $\begin{array}{c}\mathbf{R A F} \\
\mathbf{E}_{\mathbf{A}} / \mathbf{E}_{\mathbf{C}}\end{array}$ & $\begin{array}{c}\text { Risk ID } \\
\text { Bin }\end{array}$ & Media of concern & $\begin{array}{c}\text { Relative } \\
\text { Rank }\end{array}$ & $\begin{array}{c}L_{\mathrm{A}} \\
\mathbf{k m}\end{array}$ \\
\hline 1 & $50-00-0$ & $2.06 \mathrm{E}+03$ & $1.64 \mathrm{E}+08$ & $1.25 \mathrm{E}-05$ & $\mathrm{E}$ & Aquatic mammal & 40 & $6.39 \mathrm{E}+02$ \\
\hline 2 & $50-32-8$ & $2.41 E+01$ & $4.67 E+00$ & $5.15 \mathrm{E}+00$ & $\mathrm{~B}$ & Aquatic mammal & 6 & $3.24 \mathrm{E}+0 \mathrm{C}$ \\
\hline 3 & $51-28-5$ & $3.16 \mathrm{E}+03$ & $3.15 \mathrm{E}+05$ & $1.00 \mathrm{E}-02$ & $\mathrm{C}$ & Avian scavenger & 17 & $1.42 \mathrm{E}-03$ \\
\hline 4 & $67-56-1$ & $2.91 \mathrm{E}+04$ & $1.32 \mathrm{E}+09$ & $2.19 \mathrm{E}-05$ & $\mathrm{E}$ & Avian scavenger & 38 & $3.65 \mathrm{E}+02$ \\
\hline 5 & $67-63-0$ & $2.43 \mathrm{E}+03$ & $4.13 E+09$ & $5.89 \mathrm{E}-07$ & $\mathrm{~F}$ & Avian scavenger & 45 & $3.65 \mathrm{E}+02$ \\
\hline 6 & $67-66-3$ & $7.05 \mathrm{E}+01$ & $1.07 \mathrm{E}+11$ & $6.61 \mathrm{E}-10$ & $\mathrm{G}$ & Aquatic mammal & 67 & $4.52 \mathrm{E}+04$ \\
\hline 7 & $67-72-1$ & $3.30 \mathrm{E}-02$ & $5.61 \mathrm{E}+08$ & $5.89 \mathrm{E}-11$ & G & Aquatic mammal & 69 & $2.28 \mathrm{E}+05$ \\
\hline 8 & $68-12-2$ & $1.37 \mathrm{E}+01$ & $6.27 \mathrm{E}+08$ & $2.19 \mathrm{E}-08$ & $\mathrm{~F}$ & Avian scavenger & 58 & $1.40 \mathrm{E}-06$ \\
\hline 9 & $70-30-4$ & $3.16 \mathrm{E}+01$ & $5.05 \mathrm{E}+00$ & $6.26 \mathrm{E}+00$ & $\mathrm{~B}$ & Aquatic mammal & 4 & $1.79 \mathrm{E}-03$ \\
\hline 10 & $71-36-3$ & $9.46 \mathrm{E}+02$ & $2.02 \mathrm{E}+09$ & $4.68 \mathrm{E}-07$ & $\mathrm{~F}$ & Aquatic mammal & 47 & $3.04 \mathrm{E}+02$ \\
\hline 11 & $71-43-2$ & $1.54 \mathrm{E}+03$ & $2.37 \mathrm{E}+10$ & $6.48 \mathrm{E}-08$ & $\mathrm{~F}$ & Aquatic mammal & 53 & $2.71 \mathrm{E}+03$ \\
\hline 12 & $74-85-1$ & $1.90 \mathrm{E}+03$ & $3.15 \mathrm{E}+11$ & $6.02 \mathrm{E}-09$ & $\mathrm{G}$ & Aquatic mammal & 62 & $5.25 \mathrm{E}+02$ \\
\hline 13 & $76-60-8$ & $3.16 \mathrm{E}-01$ & $2.04 \mathrm{E}+02$ & $1.55 \mathrm{E}-03$ & $\mathrm{D}$ & Aquatic mammal & 23 & $1.91 \mathrm{E}-02$ \\
\hline 14 & $77-54-3$ & $3.16 \mathrm{E}+01$ & $2.23 \mathrm{E}+05$ & $1.42 \mathrm{E}-04$ & $\mathrm{D}$ & Terrestrial carnivore & 34 & $3.66 \mathrm{E}+02$ \\
\hline 15 & $78-87-5$ & $3.16 \mathrm{E}+03$ & $2.26 \mathrm{E}+10$ & $1.40 \mathrm{E}-07$ & $\mathrm{~F}$ & Aquatic mammal & 52 & $1.17 \mathrm{E}+04$ \\
\hline 16 & $78-93-3$ & $3.49 \mathrm{E}+03$ & $2.05 \mathrm{E}+10$ & $1.70 \mathrm{E}-07$ & $\mathrm{~F}$ & Aquatic mammal & 51 & $3.12 \mathrm{E}+03$ \\
\hline 17 & $79-01-6$ & $7.30 \mathrm{E}+02$ & $4.12 E+09$ & $1.77 \mathrm{E}-07$ & $\mathrm{~F}$ & Aquatic mammal & 50 & $6.58 \mathrm{E}+03$ \\
\hline 18 & $79-34-5$ & $3.14 \mathrm{E}-01$ & $6.94 \mathrm{E}+09$ & $4.52 \mathrm{E}-11$ & $\mathrm{G}$ & Aquatic mammal & 70 & $2.41 \mathrm{E}+04$ \\
\hline 19 & $79-92-5$ & $3.16 \mathrm{E}+01$ & $4.16 \mathrm{E}+10$ & $7.59 \mathrm{E}-10$ & $\mathrm{G}$ & Aquatic mammal & 65 & $7.91 \mathrm{E}+01$ \\
\hline 20 & $79-94-7$ & $3.16 \mathrm{E}+03$ & $5.49 \mathrm{E}+02$ & $5.76 \mathrm{E}+00$ & $\mathrm{~B}$ & Aquatic mammal & 5 & 4.16E-03 \\
\hline 21 & $84-74-2$ & $6.78 \mathrm{E}+00$ & $2.53 \mathrm{E}+05$ & $2.68 \mathrm{E}-05$ & $\mathrm{E}$ & Terrestrial carnivore & 36 & $2.95 \mathrm{E}+0 \mathrm{C}$ \\
\hline 22 & $85-01-8$ & $1.71 \mathrm{E}+02$ & $1.13 \mathrm{E}+04$ & $1.52 \mathrm{E}-02$ & $\mathrm{C}$ & Terrestrial carnivore & 15 & $3.49 \mathrm{E}+02$ \\
\hline 23 & $85-68-7$ & $2.09 \mathrm{E}+01$ & $2.22 \mathrm{E}+05$ & $9.39 \mathrm{E}-05$ & $\mathrm{E}$ & Terrestrial carnivore & 35 & $1.21 \mathrm{E}+01$ \\
\hline
\end{tabular}




\begin{tabular}{|c|c|c|c|c|c|c|c|c|}
\hline ID \# & CAS \# & $\begin{array}{c}\mathbf{E}_{\mathrm{A}} \\
\text { t / year }\end{array}$ & $\begin{array}{l}\mathrm{L} \text { II } \mathrm{E}_{\mathrm{C}} \\
\text { t / year }\end{array}$ & $\begin{array}{c}\mathbf{R A F} \\
\mathbf{E}_{\mathbf{A}} / \mathbf{E}_{\mathbf{C}}\end{array}$ & $\begin{array}{c}\text { Risk ID } \\
\text { Bin }\end{array}$ & Media of concern & $\begin{array}{c}\text { Relative } \\
\text { Rank }\end{array}$ & $\begin{array}{r}L_{\mathrm{A}} \\
\mathbf{k m}\end{array}$ \\
\hline 24 & $87-86-5$ & $3.16 \mathrm{E}+03$ & $2.49 \mathrm{E}+02$ & $1.27 \mathrm{E}+01$ & $\mathrm{~B}$ & Terrestrial carnivore & 3 & $1.06 \mathrm{E}+02$ \\
\hline 25 & $91-20-3$ & $2.78 \mathrm{E}+02$ & $1.09 \mathrm{E}+06$ & $2.55 \mathrm{E}-04$ & $\mathrm{D}$ & Terrestrial carnivore & 31 & $2.39 \mathrm{E}+02$ \\
\hline 26 & $95-50-1$ & $1.10 \mathrm{E}+01$ & $8.13 \mathrm{E}+08$ & $1.35 \mathrm{E}-08$ & $\mathrm{~F}$ & Aquatic mammal & 59 & $1.27 \mathrm{E}+04$ \\
\hline 27 & $95-63-6$ & $9.85 \mathrm{E}+02$ & $2.20 \mathrm{E}+09$ & $4.48 \mathrm{E}-07$ & $\mathrm{~F}$ & Aquatic mammal & 48 & $3.19 \mathrm{E}+02$ \\
\hline 28 & $101-61-1$ & $3.16 \mathrm{E}-01$ & $4.23 \mathrm{E}+04$ & $7.48 \mathrm{E}-06$ & $\mathrm{E}$ & Terrestrial carnivore & 41 & $1.18 \mathrm{E}+01$ \\
\hline 29 & $104-40-5$ & $1.93 E+02$ & $1.08 \mathrm{E}+03$ & $1.78 \mathrm{E}-01$ & $\mathrm{C}$ & Aquatic mammal & 13 & $4.75 \mathrm{E}+01$ \\
\hline 30 & $106-46-7$ & $1.60 \mathrm{E}+01$ & $5.64 \mathrm{E}+08$ & $2.84 \mathrm{E}-08$ & $\mathrm{~F}$ & Aquatic mammal & 56 & $1.27 \mathrm{E}+04$ \\
\hline 31 & $107-06-2$ & $9.52 \mathrm{E}+00$ & $2.23 \mathrm{E}+10$ & $4.27 \mathrm{E}-10$ & G & Aquatic mammal & 68 & $1.92 \mathrm{E}+04$ \\
\hline 32 & $107-21-1$ & $3.64 \mathrm{E}+03$ & $1.32 \mathrm{E}+09$ & $2.75 \mathrm{E}-06$ & $\mathrm{E}$ & Avian scavenger & 43 & $3.06 \mathrm{E}-01$ \\
\hline 33 & $108-10-1$ & $7.52 \mathrm{E}+02$ & $2.53 \mathrm{E}+10$ & $2.97 \mathrm{E}-08$ & $\mathrm{~F}$ & Aquatic mammal & 55 & $5.93 \mathrm{E}+02$ \\
\hline 34 & $108-88-3$ & $7.27 \mathrm{E}+03$ & $1.36 \mathrm{E}+10$ & $5.35 \mathrm{E}-07$ & $\mathrm{~F}$ & Aquatic mammal & 46 & $1.02 \mathrm{E}+03$ \\
\hline 35 & $108-90-7$ & $4.56 \mathrm{E}+00$ & $5.34 \mathrm{E}+09$ & $8.55 \mathrm{E}-10$ & G & Aquatic mammal & 64 & $3.85 \mathrm{E}+03$ \\
\hline 36 & $110-54-3$ & $6.04 \mathrm{E}+03$ & $2.70 \mathrm{E}+11$ & $2.24 \mathrm{E}-08$ & $\mathrm{~F}$ & Aquatic mammal & 57 & $9.77 \mathrm{E}+02$ \\
\hline 37 & $110-82-7$ & $1.64 \mathrm{E}+03$ & $1.39 \mathrm{E}+11$ & $1.18 \mathrm{E}-08$ & $\mathrm{~F}$ & Aquatic mammal & 60 & $6.28 \mathrm{E}+02$ \\
\hline 38 & $111-42-2$ & $3.55 \mathrm{E}+02$ & $1.16 \mathrm{E}+08$ & $3.05 \mathrm{E}-06$ & $\mathrm{E}$ & Avian scavenger & 42 & $1.58 \mathrm{E}-03$ \\
\hline 39 & $115-07-1$ & $1.11 \mathrm{E}+03$ & $1.55 \mathrm{E}+12$ & $7.17 \mathrm{E}-10$ & G & Aquatic mammal & 66 & $1.42 \mathrm{E}+02$ \\
\hline 40 & $117-81-7$ & $8.02 \mathrm{E}+01$ & $6.65 \mathrm{E}+04$ & $1.21 \mathrm{E}-03$ & $\mathrm{D}$ & Aquatic mammal & 25 & $3.77 \mathrm{E}+01$ \\
\hline 41 & $118-74-1$ & $2.95 \mathrm{E}-02$ & $1.42 \mathrm{E}+05$ & $2.08 \mathrm{E}-07$ & $\mathrm{~F}$ & Aquatic mammal & 49 & $6.49 \mathrm{E}+04$ \\
\hline 42 & $120-12-7$ & $1.22 \mathrm{E}+01$ & $1.78 \mathrm{E}+01$ & $6.84 \mathrm{E}-01$ & $\mathrm{C}$ & Terrestrial carnivore & 8 & $3.98 \mathrm{E}+01$ \\
\hline 43 & $120-83-2$ & $3.16 \mathrm{E}+01$ & $3.01 \mathrm{E}+04$ & $1.05 \mathrm{E}-03$ & $\mathrm{D}$ & Terrestrial carnivore & 27 & $1.38 \mathrm{E}+03$ \\
\hline 44 & $124-18-5$ & $3.16 \mathrm{E}+01$ & $4.71 \mathrm{E}+09$ & $6.71 \mathrm{E}-09$ & $\mathrm{G}$ & Fish & 61 & $4.79 \mathrm{E}+02$ \\
\hline 45 & $127-18-4$ & $4.21 \mathrm{E}+01$ & $1.94 \mathrm{E}+10$ & $2.17 \mathrm{E}-09$ & $\mathrm{G}$ & Aquatic mammal & 63 & $2.48 \mathrm{E}+04$ \\
\hline 46 & $129-00-0$ & $9.37 \mathrm{E}+01$ & $2.94 \mathrm{E}+00$ & $3.19 \mathrm{E}+01$ & $\mathrm{~B}$ & Terrestrial carnivore & 1 & $7.81 \mathrm{E}+01$ \\
\hline 47 & $603-35-0$ & $3.16 \mathrm{E}+01$ & $3.36 \mathrm{E}+04$ & $9.41 \mathrm{E}-04$ & $\mathrm{D}$ & Aquatic mammal & 28 & $2.01 \mathrm{E}+01$ \\
\hline
\end{tabular}




\begin{tabular}{|c|c|c|c|c|c|c|c|c|}
\hline ID \# & CAS \# & $\begin{array}{c}\mathbf{E}_{\mathbf{A}} \\
\text { t / year }\end{array}$ & $\begin{array}{l}\text { L II E } E_{C} \\
\text { t / year }\end{array}$ & $\begin{array}{c}\mathbf{R A F} \\
\mathbf{E}_{\mathbf{A}} / \mathbf{E}_{\mathbf{C}}\end{array}$ & $\begin{array}{c}\text { Risk ID } \\
\text { Bin }\end{array}$ & Media of concern & $\begin{array}{c}\text { Relative } \\
\text { Rank }\end{array}$ & $\begin{array}{r}L_{\mathrm{A}} \\
\mathbf{k m}\end{array}$ \\
\hline 48 & $608-93-5$ & $3.16 \mathrm{E}-01$ & $2.56 \mathrm{E}+05$ & $1.23 \mathrm{E}-06$ & $\mathrm{E}$ & Terrestrial carnivore & 44 & $2.63 \mathrm{E}+04$ \\
\hline 49 & $1163-19-5$ & $5.77 \mathrm{E}+00$ & $9.93 \mathrm{E}+03$ & $5.81 \mathrm{E}-04$ & $\mathrm{D}$ & Terrestrial carnivore & 30 & $1.27 \mathrm{E}-02$ \\
\hline 50 & $1222-05-5$ & $3.16 \mathrm{E}+01$ & $2.70 \mathrm{E}+04$ & $1.17 \mathrm{E}-03$ & $\mathrm{D}$ & Aquatic mammal & 26 & $1.17 \mathrm{E}+02$ \\
\hline 51 & $1330-78-5$ & $3.16 \mathrm{E}+03$ & $3.50 \mathrm{E}+04$ & $9.03 \mathrm{E}-02$ & $\mathrm{C}$ & Aquatic mammal & 14 & $4.78 \mathrm{E}+00$ \\
\hline 52 & $1582-09-8$ & $3.16 \mathrm{E}-01$ & $1.70 \mathrm{E}+03$ & $1.86 \mathrm{E}-04$ & $\mathrm{D}$ & Terrestrial carnivore & 32 & $1.85 \mathrm{E}+02$ \\
\hline 53 & $1897-45-6$ & $3.16 \mathrm{E}+01$ & $2.10 \mathrm{E}+01$ & $1.51 \mathrm{E}+00$ & $\mathrm{~B}$ & Terrestrial carnivore & 7 & $2.29 \mathrm{E}+00$ \\
\hline 54 & $1912-24-9$ & $3.16 \mathrm{E}+01$ & $1.25 \mathrm{E}+04$ & $2.54 \mathrm{E}-03$ & $\mathrm{D}$ & Avian scavenger & 21 & $1.13 \mathrm{E}-05$ \\
\hline 55 & $3380-34-5$ & $3.16 \mathrm{E}+01$ & $1.29 \mathrm{E}+00$ & $2.45 \mathrm{E}+01$ & $\mathrm{~B}$ & Terrestrial carnivore & 2 & $1.38 \mathrm{E}+01$ \\
\hline 56 & $3846-71-7$ & $3.16 \mathrm{E}+01$ & $7.44 \mathrm{E}+03$ & $4.25 \mathrm{E}-03$ & $\mathrm{D}$ & Aquatic mammal & 20 & $4.69 \mathrm{E}-03$ \\
\hline 57 & $4979-32-2$ & $3.16 \mathrm{E}+01$ & $2.15 \mathrm{E}+05$ & $1.47 \mathrm{E}-04$ & $\mathrm{D}$ & Terrestrial carnivore & 33 & $1.44 \mathrm{E}-01$ \\
\hline 58 & $5285-60-9$ & $3.16 \mathrm{E}+03$ & $1.70 \mathrm{E}+04$ & $1.86 \mathrm{E}-01$ & $\mathrm{C}$ & Aquatic mammal & 12 & $4.23 \mathrm{E}+00$ \\
\hline 59 & $6386-38-5$ & $3.16 \mathrm{E}+01$ & $3.56 \mathrm{E}+04$ & $8.88 \mathrm{E}-04$ & $\mathrm{D}$ & Terrestrial carnivore & 29 & $1.91 \mathrm{E}+00$ \\
\hline 60 & $13560-89-9$ & $3.16 \mathrm{E}+01$ & $4.30 \mathrm{E}+03$ & $7.36 \mathrm{E}-03$ & $\mathrm{D}$ & Terrestrial carnivore & 18 & $1.29 \mathrm{E}+00$ \\
\hline 61 & $15646-96-5$ & $3.16 \mathrm{E}+01$ & $2.01 \mathrm{E}+06$ & $1.57 \mathrm{E}-05$ & $\mathrm{E}$ & Terrestrial carnivore & 39 & $5.27 \mathrm{E}+02$ \\
\hline 62 & $23593-75-1$ & $3.16 \mathrm{E}-01$ & $1.22 \mathrm{E}+04$ & $2.59 \mathrm{E}-05$ & $\mathrm{E}$ & Terrestrial carnivore & 37 & $6.35 \mathrm{E}-02$ \\
\hline 63 & $25973-55-1$ & $3.16 \mathrm{E}+01$ & $5.71 \mathrm{E}+03$ & $5.54 \mathrm{E}-03$ & $\mathrm{D}$ & Aquatic mammal & 19 & $1.13 \mathrm{E}-03$ \\
\hline 64 & $26140-60-3$ & $3.16 \mathrm{E}+01$ & $1.59 \mathrm{E}+04$ & $1.99 \mathrm{E}-03$ & $\mathrm{D}$ & Aquatic mammal & 22 & $4.80 \mathrm{E}-01$ \\
\hline 65 & $32534-81-9$ & $3.16 \mathrm{E}+01$ & $2.53 \mathrm{E}+03$ & $1.25 \mathrm{E}-02$ & $\mathrm{C}$ & Aquatic mammal & 16 & $6.70 \mathrm{E}+01$ \\
\hline 66 & $32536-52-0$ & $3.16 \mathrm{E}+03$ & $5.23 \mathrm{E}+03$ & $6.04 \mathrm{E}-01$ & $\mathrm{C}$ & Terrestrial carnivore & 9 & $8.31 \mathrm{E}-02$ \\
\hline 67 & $54079-53-7$ & $3.16 \mathrm{E}+01$ & $2.36 \mathrm{E}+04$ & $1.34 \mathrm{E}-03$ & $\mathrm{D}$ & Aquatic mammal & 24 & $4.03 \mathrm{E}-05$ \\
\hline 68 & $54827-17-7$ & $3.16 \mathrm{E}-01$ & $5.07 \mathrm{E}+06$ & $6.23 \mathrm{E}-08$ & $\mathrm{~F}$ & Aquatic mammal & 54 & $5.37 \mathrm{E}-03$ \\
\hline 69 & $63936-56-1$ & $3.16 \mathrm{E}+03$ & $5.75 \mathrm{E}+03$ & $5.50 \mathrm{E}-01$ & $\mathrm{C}$ & Terrestrial carnivore & 10 & $1.86 \mathrm{E}-02$ \\
\hline 70 & 89347-09-1 & $3.16 \mathrm{E}+03$ & $1.20 \mathrm{E}+04$ & $2.64 \mathrm{E}-01$ & $\mathrm{C}$ & Terrestrial carnivore & 11 & $1.26 \mathrm{E}-03$ \\
\hline
\end{tabular}


Table SI - 11. Summary RAIDAR Level III model output for 70 chemicals based on unit emissions to air.

\begin{tabular}{|c|c|c|c|c|c|c|c|c|}
\hline ID \# & CAS \# & $\begin{array}{c}\mathbf{E}_{\mathrm{A}} \\
\mathrm{t} / \text { year }\end{array}$ & $\begin{array}{c}\text { L III-Air } E_{C} \\
t / \text { year }\end{array}$ & $\begin{array}{c}\mathbf{R A F} \\
\mathbf{E}_{\mathbf{A}} / \mathbf{E}_{\mathbf{C}}\end{array}$ & $\begin{array}{c}\text { Risk ID } \\
\text { Bin }\end{array}$ & Media of concern & $\begin{array}{c}\text { Relative } \\
\text { Rank }\end{array}$ & $\begin{array}{r}L_{\mathrm{A}} \\
\mathbf{k m}\end{array}$ \\
\hline 1 & $50-00-0$ & $2.06 \mathrm{E}+03$ & $1.63 \mathrm{E}+08$ & $1.26 \mathrm{E}-05$ & E & Aquatic mammal & 45 & $6.51 \mathrm{E}+02$ \\
\hline 2 & $50-32-8$ & $2.41 \mathrm{E}+01$ & $3.83 \mathrm{E}-01$ & $6.28 \mathrm{E}+01$ & $\mathrm{~B}$ & Terrestrial carnivore & 10 & $1.01 \mathrm{E}+02$ \\
\hline 3 & $51-28-5$ & $3.16 \mathrm{E}+03$ & $4.20 \mathrm{E}+00$ & $7.52 \mathrm{E}+02$ & A & Foliage vegetation & 4 & $5.48 \mathrm{E}+02$ \\
\hline 4 & $67-56-1$ & $2.91 E+04$ & $4.76 \mathrm{E}+08$ & $6.10 \mathrm{E}-05$ & $\mathrm{E}$ & Avian & 42 & $5.43 \mathrm{E}+03$ \\
\hline 5 & $67-63-0$ & $2.43 \mathrm{E}+03$ & $3.39 \mathrm{E}+09$ & $7.17 \mathrm{E}-07$ & $\mathrm{~F}$ & Avian & 47 & $7.06 \mathrm{E}+02$ \\
\hline 6 & $67-66-3$ & $7.05 \mathrm{E}+01$ & $1.07 \mathrm{E}+11$ & $6.61 \mathrm{E}-10$ & $\mathrm{G}$ & Aquatic mammal & 67 & $4.53 \mathrm{E}+04$ \\
\hline 7 & $67-72-1$ & $3.30 \mathrm{E}-02$ & $5.41 \mathrm{E}+08$ & $6.10 \mathrm{E}-11$ & G & Aquatic mammal & 69 & $2.31 \mathrm{E}+05$ \\
\hline 8 & $68-12-2$ & $1.37 \mathrm{E}+01$ & $1.57 \mathrm{E}+01$ & $8.75 \mathrm{E}-01$ & $\mathrm{C}$ & Foliage vegetation & 19 & $2.45 \mathrm{E}+02$ \\
\hline 9 & $70-30-4$ & $3.16 \mathrm{E}+01$ & $1.19 \mathrm{E}-02$ & $2.65 \mathrm{E}+03$ & A & Terrestrial carnivore & 3 & $4.17 \mathrm{E}+02$ \\
\hline 10 & $71-36-3$ & $9.46 \mathrm{E}+02$ & $1.42 \mathrm{E}+09$ & $6.66 \mathrm{E}-07$ & $\mathrm{~F}$ & Aquatic mammal & 48 & $7.43 \mathrm{E}+02$ \\
\hline 11 & $71-43-2$ & $1.54 \mathrm{E}+03$ & $2.38 \mathrm{E}+10$ & $6.47 \mathrm{E}-08$ & $\mathrm{~F}$ & Aquatic mammal & 55 & $2.72 \mathrm{E}+03$ \\
\hline 12 & $74-85-1$ & $1.90 \mathrm{E}+03$ & $3.15 \mathrm{E}+11$ & $6.01 \mathrm{E}-09$ & G & Aquatic mammal & 61 & $5.25 \mathrm{E}+02$ \\
\hline 13 & $76-60-8$ & $3.16 \mathrm{E}-01$ & $5.29 \mathrm{E}-01$ & $5.97 \mathrm{E}-01$ & $\mathrm{C}$ & Terrestrial carnivore & 20 & $3.67 \mathrm{E}+02$ \\
\hline 14 & $77-54-3$ & $3.16 \mathrm{E}+01$ & $2.38 \mathrm{E}+05$ & $1.33 \mathrm{E}-04$ & $\mathrm{D}$ & Terrestrial carnivore & 40 & $4.46 \mathrm{E}+02$ \\
\hline 15 & $78-87-5$ & $3.16 \mathrm{E}+03$ & $2.26 \mathrm{E}+10$ & $1.40 \mathrm{E}-07$ & $\mathrm{~F}$ & Aquatic mammal & 54 & $1.17 \mathrm{E}+04$ \\
\hline 16 & $78-93-3$ & $3.49 \mathrm{E}+03$ & $1.98 \mathrm{E}+10$ & $1.76 \mathrm{E}-07$ & $\mathrm{~F}$ & Aquatic mammal & 53 & $3.69 \mathrm{E}+03$ \\
\hline 17 & 79-01-6 & $7.30 \mathrm{E}+02$ & $4.12 \mathrm{E}+09$ & $1.77 \mathrm{E}-07$ & $\mathrm{~F}$ & Aquatic mammal & 52 & $6.58 \mathrm{E}+03$ \\
\hline 18 & $79-34-5$ & $3.14 \mathrm{E}-01$ & $7.01 \mathrm{E}+09$ & $4.48 \mathrm{E}-11$ & G & Aquatic mammal & 70 & $2.43 \mathrm{E}+04$ \\
\hline 19 & $79-92-5$ & $3.16 \mathrm{E}+01$ & $4.28 \mathrm{E}+10$ & $7.38 \mathrm{E}-10$ & G & Aquatic mammal & 65 & $7.91 \mathrm{E}+01$ \\
\hline 20 & $79-94-7$ & $3.16 \mathrm{E}+03$ & $6.91 \mathrm{E}-01$ & $4.58 \mathrm{E}+03$ & A & Terrestrial carnivore & 2 & $3.87 \mathrm{E}+02$ \\
\hline 21 & $84-74-2$ & $6.78 \mathrm{E}+00$ & $2.57 \mathrm{E}+03$ & $2.63 \mathrm{E}-03$ & $\mathrm{D}$ & Terrestrial carnivore & 32 & $4.86 \mathrm{E}+02$ \\
\hline 22 & $85-01-8$ & $1.71 \mathrm{E}+02$ & $1.24 \mathrm{E}+04$ & $1.38 \mathrm{E}-02$ & $\mathrm{C}$ & Terrestrial carnivore & 30 & $4.06 \mathrm{E}+02$ \\
\hline 23 & $85-68-7$ & $2.09 \mathrm{E}+01$ & $9.53 \mathrm{E}+03$ & $2.19 \mathrm{E}-03$ & $\mathrm{D}$ & Terrestrial carnivore & 34 & $4.62 \mathrm{E}+02$ \\
\hline
\end{tabular}




\begin{tabular}{|c|c|c|c|c|c|c|c|c|}
\hline ID \# & CAS \# & $\begin{array}{c}\mathbf{E}_{\mathrm{A}} \\
\text { t / year }\end{array}$ & $\begin{array}{c}\text { L III-Air } E_{C} \\
\text { t / year }\end{array}$ & $\begin{array}{c}\mathbf{R A F} \\
\mathbf{E}_{\mathbf{A}} / \mathbf{E}_{\mathbf{C}}\end{array}$ & $\begin{array}{c}\text { Risk ID } \\
\text { Bin }\end{array}$ & Media of concern & $\begin{array}{c}\text { Relative } \\
\text { Rank }\end{array}$ & $\begin{array}{r}L_{\mathrm{A}} \\
\mathbf{k m}\end{array}$ \\
\hline 24 & $87-86-5$ & $3.16 \mathrm{E}+03$ & $3.05 \mathrm{E}+01$ & $1.04 \mathrm{E}+02$ & A & Terrestrial carnivore & 9 & $3.54 \mathrm{E}+03$ \\
\hline 25 & $91-20-3$ & $2.78 \mathrm{E}+02$ & $1.26 \mathrm{E}+06$ & $2.22 \mathrm{E}-04$ & $\mathrm{D}$ & Terrestrial carnivore & 38 & $2.45 \mathrm{E}+02$ \\
\hline 26 & $95-50-1$ & $1.10 \mathrm{E}+01$ & $8.37 \mathrm{E}+08$ & $1.31 \mathrm{E}-08$ & $\mathrm{~F}$ & Aquatic mammal & 59 & $1.28 \mathrm{E}+04$ \\
\hline 27 & $95-63-6$ & $9.85 \mathrm{E}+02$ & $2.44 \mathrm{E}+09$ & 4.04E-07 & $\mathrm{F}$ & Aquatic mammal & 50 & $3.19 \mathrm{E}+02$ \\
\hline 28 & 101-61-1 & $3.16 \mathrm{E}-01$ & $2.48 \mathrm{E}+04$ & $1.27 \mathrm{E}-05$ & $\mathrm{E}$ & Terrestrial carnivore & 44 & $2.53 \mathrm{E}+01$ \\
\hline 29 & $104-40-5$ & $1.93 \mathrm{E}+02$ & $9.02 \mathrm{E}+02$ & $2.14 \mathrm{E}-01$ & $\mathrm{C}$ & Terrestrial carnivore & 24 & $1.03 \mathrm{E}+02$ \\
\hline 30 & $106-46-7$ & $1.60 \mathrm{E}+01$ & $5.85 \mathrm{E}+08$ & $2.73 \mathrm{E}-08$ & $\mathrm{~F}$ & Aquatic mammal & 57 & $1.28 \mathrm{E}+04$ \\
\hline 31 & $107-06-2$ & $9.52 \mathrm{E}+00$ & $2.23 \mathrm{E}+10$ & $4.26 \mathrm{E}-10$ & $\mathrm{G}$ & Aquatic mammal & 68 & $1.94 \mathrm{E}+04$ \\
\hline 32 & $107-21-1$ & $3.64 E+03$ & $6.00 \mathrm{E}+06$ & $6.06 \mathrm{E}-04$ & $\mathrm{D}$ & Foliage vegetation & 36 & $3.08 \mathrm{E}+02$ \\
\hline 33 & $108-10-1$ & $7.52 \mathrm{E}+02$ & $2.55 \mathrm{E}+10$ & $2.95 \mathrm{E}-08$ & $\mathrm{~F}$ & Aquatic mammal & 56 & $5.98 \mathrm{E}+02$ \\
\hline 34 & $108-88-3$ & $7.27 \mathrm{E}+03$ & $1.37 \mathrm{E}+10$ & $5.30 \mathrm{E}-07$ & $\mathrm{~F}$ & Aquatic mammal & 49 & $1.02 \mathrm{E}+03$ \\
\hline 35 & $108-90-7$ & $4.56 \mathrm{E}+00$ & $5.38 \mathrm{E}+09$ & $8.48 \mathrm{E}-10$ & $\mathrm{G}$ & Aquatic mammal & 64 & $3.85 \mathrm{E}+03$ \\
\hline 36 & $110-54-3$ & $6.04 \mathrm{E}+03$ & $2.70 \mathrm{E}+11$ & $2.24 \mathrm{E}-08$ & $\mathrm{~F}$ & Aquatic mammal & 58 & $9.77 \mathrm{E}+02$ \\
\hline 37 & $110-82-7$ & $1.64 \mathrm{E}+03$ & $1.39 \mathrm{E}+11$ & $1.18 \mathrm{E}-08$ & $\mathrm{~F}$ & Aquatic mammal & 60 & $6.28 \mathrm{E}+02$ \\
\hline 38 & $111-42-2$ & $3.55 \mathrm{E}+02$ & $1.37 \mathrm{E}+04$ & $2.59 \mathrm{E}-02$ & $\mathrm{C}$ & Foliage vegetation & 28 & $5.23 \mathrm{E}+01$ \\
\hline 39 & $115-07-1$ & $1.11 \mathrm{E}+03$ & $1.55 \mathrm{E}+12$ & $7.17 \mathrm{E}-10$ & G & Aquatic mammal & 66 & $1.42 \mathrm{E}+02$ \\
\hline 40 & $117-81-7$ & $8.02 \mathrm{E}+01$ & $3.35 \mathrm{E}+04$ & $2.39 \mathrm{E}-03$ & $\mathrm{D}$ & Terrestrial carnivore & 33 & $2.43 \mathrm{E}+02$ \\
\hline 41 & $118-74-1$ & $2.95 \mathrm{E}-02$ & $8.85 \mathrm{E}+04$ & $3.33 \mathrm{E}-07$ & $\mathrm{~F}$ & Aquatic mammal & 51 & $1.49 \mathrm{E}+05$ \\
\hline 42 & $120-12-7$ & $1.22 \mathrm{E}+01$ & $7.24 \mathrm{E}+00$ & $1.68 \mathrm{E}+00$ & $\mathrm{~B}$ & Terrestrial carnivore & 16 & $1.29 \mathrm{E}+02$ \\
\hline 43 & $120-83-2$ & $3.16 \mathrm{E}+01$ & $3.25 E+04$ & $9.74 \mathrm{E}-04$ & $\mathrm{D}$ & Terrestrial carnivore & 35 & $1.68 \mathrm{E}+03$ \\
\hline 44 & $124-18-5$ & $3.16 \mathrm{E}+01$ & $7.79 \mathrm{E}+09$ & $4.06 \mathrm{E}-09$ & G & Aquatic mammal & 62 & $4.79 \mathrm{E}+02$ \\
\hline 45 & $127-18-4$ & $4.21 \mathrm{E}+01$ & $1.94 \mathrm{E}+10$ & $2.17 \mathrm{E}-09$ & G & Aquatic mammal & 63 & $2.48 \mathrm{E}+04$ \\
\hline 46 & $129-00-0$ & $9.37 \mathrm{E}+01$ & $2.75 \mathrm{E}+00$ & $3.40 \mathrm{E}+01$ & $\mathrm{~B}$ & Terrestrial carnivore & 11 & $1.06 \mathrm{E}+02$ \\
\hline 47 & $603-35-0$ & $3.16 \mathrm{E}+01$ & $1.97 \mathrm{E}+03$ & $1.61 \mathrm{E}-02$ & $\mathrm{C}$ & Terrestrial carnivore & 29 & $8.71 \mathrm{E}+02$ \\
\hline
\end{tabular}




\begin{tabular}{|c|c|c|c|c|c|c|c|c|}
\hline ID \# & CAS \# & $\begin{array}{c}\mathrm{E}_{\mathrm{A}} \\
\text { t / year }\end{array}$ & $\begin{array}{c}\text { L III-Air } E_{C} \\
\text { t / year }\end{array}$ & $\begin{array}{c}\mathbf{R A F} \\
\mathbf{E}_{\mathbf{A}} / \mathbf{E}_{\mathbf{C}}\end{array}$ & $\begin{array}{c}\text { Risk ID } \\
\text { Bin }\end{array}$ & Media of concern & $\begin{array}{c}\text { Relative } \\
\text { Rank }\end{array}$ & $\begin{array}{r}L_{\mathrm{A}} \\
\mathbf{k m}\end{array}$ \\
\hline 48 & $608-93-5$ & $3.16 \mathrm{E}-01$ & $1.96 \mathrm{E}+05$ & $1.61 \mathrm{E}-06$ & E & Aquatic mammal & 46 & $6.64 \mathrm{E}+04$ \\
\hline 49 & $1163-19-5$ & $5.77 \mathrm{E}+00$ & $2.24 \mathrm{E}+01$ & $2.57 \mathrm{E}-01$ & $\mathrm{C}$ & Terrestrial carnivore & 23 & $5.22 \mathrm{E}+02$ \\
\hline 50 & $1222-05-5$ & $3.16 \mathrm{E}+01$ & $5.59 \mathrm{E}+04$ & $5.66 \mathrm{E}-04$ & $\mathrm{D}$ & Terrestrial carnivore & 37 & $1.41 \mathrm{E}+02$ \\
\hline 51 & $1330-78-5$ & $3.16 \mathrm{E}+03$ & $1.40 \mathrm{E}+03$ & $2.26 \mathrm{E}+00$ & $\mathrm{~B}$ & Terrestrial carnivore & 14 & $3.81 \mathrm{E}+02$ \\
\hline 52 & $1582-09-8$ & $3.16 \mathrm{E}-01$ & $1.81 \mathrm{E}+03$ & $1.74 \mathrm{E}-04$ & $\mathrm{D}$ & Terrestrial carnivore & 39 & $2.21 \mathrm{E}+02$ \\
\hline 53 & $1897-45-6$ & $3.16 \mathrm{E}+01$ & $1.49 \mathrm{E}-01$ & $2.12 \mathrm{E}+02$ & A & Terrestrial carnivore & 6 & $5.86 \mathrm{E}+02$ \\
\hline 54 & $1912-24-9$ & $3.16 \mathrm{E}+01$ & $4.04 \mathrm{E}-03$ & $7.82 \mathrm{E}+03$ & A & Foliage vegetation & 1 & $1.47 \mathrm{E}+02$ \\
\hline 55 & $3380-34-5$ & $3.16 \mathrm{E}+01$ & $9.39 \mathrm{E}-02$ & $3.36 \mathrm{E}+02$ & A & Terrestrial carnivore & 5 & $2.82 \mathrm{E}+02$ \\
\hline 56 & $3846-71-7$ & $3.16 \mathrm{E}+01$ & $1.36 \mathrm{E}+01$ & $2.33 \mathrm{E}+00$ & $\mathrm{~B}$ & Terrestrial carnivore & 13 & $2.20 \mathrm{E}+02$ \\
\hline 57 & $4979-32-2$ & $3.16 \mathrm{E}+01$ & $1.02 \mathrm{E}+03$ & $3.09 \mathrm{E}-02$ & $\mathrm{C}$ & Terrestrial carnivore & 27 & $4.49 \mathrm{E}+01$ \\
\hline 58 & $5285-60-9$ & $3.16 \mathrm{E}+03$ & $2.96 \mathrm{E}+03$ & $1.07 \mathrm{E}+00$ & $\mathrm{~B}$ & Terrestrial carnivore & 18 & $3.48 \mathrm{E}+01$ \\
\hline 59 & $6386-38-5$ & $3.16 \mathrm{E}+01$ & $4.52 \mathrm{E}+02$ & $6.99 \mathrm{E}-02$ & $\mathrm{C}$ & Terrestrial carnivore & 26 & $2.22 \mathrm{E}+02$ \\
\hline 60 & $13560-89-9$ & $3.16 \mathrm{E}+01$ & $7.26 \mathrm{E}+01$ & $4.35 \mathrm{E}-01$ & $\mathrm{C}$ & Terrestrial carnivore & 22 & $1.94 \mathrm{E}+02$ \\
\hline 61 & $15646-96-5$ & $3.16 \mathrm{E}+01$ & $2.18 \mathrm{E}+06$ & $1.45 \mathrm{E}-05$ & $\mathrm{E}$ & Terrestrial carnivore & 43 & $6.18 \mathrm{E}+02$ \\
\hline 62 & $23593-75-1$ & $3.16 \mathrm{E}-01$ & $4.11 \mathrm{E}+01$ & $7.69 \mathrm{E}-03$ & $\mathrm{D}$ & Terrestrial carnivore & 31 & $9.43 \mathrm{E}+01$ \\
\hline 63 & $25973-55-1$ & $3.16 \mathrm{E}+01$ & $1.56 \mathrm{E}+01$ & $2.03 \mathrm{E}+00$ & $\mathrm{~B}$ & Terrestrial carnivore & 15 & $2.01 \mathrm{E}+02$ \\
\hline 64 & $26140-60-3$ & $3.16 \mathrm{E}+01$ & $5.41 \mathrm{E}+01$ & $5.84 \mathrm{E}-01$ & $\mathrm{C}$ & Terrestrial carnivore & 21 & $4.35 \mathrm{E}+02$ \\
\hline 65 & $32534-81-9$ & $3.16 \mathrm{E}+01$ & $3.80 \mathrm{E}+02$ & $8.32 \mathrm{E}-02$ & $\mathrm{C}$ & Terrestrial carnivore & 25 & $7.59 \mathrm{E}+03$ \\
\hline 66 & $32536-52-0$ & $3.16 \mathrm{E}+03$ & $1.91 \mathrm{E}+01$ & $1.65 \mathrm{E}+02$ & A & Terrestrial carnivore & 7 & $5.54 \mathrm{E}+02$ \\
\hline 67 & $54079-53-7$ & $3.16 \mathrm{E}+01$ & $1.89 \mathrm{E}+01$ & $1.67 \mathrm{E}+00$ & $\mathrm{~B}$ & Terrestrial carnivore & 17 & $1.98 \mathrm{E}+01$ \\
\hline 68 & $54827-17-7$ & $3.16 \mathrm{E}-01$ & $4.25 \mathrm{E}+03$ & $7.43 \mathrm{E}-05$ & $\mathrm{E}$ & Human & 41 & $6.22 \mathrm{E}+01$ \\
\hline 69 & $63936-56-1$ & $3.16 \mathrm{E}+03$ & $2.06 \mathrm{E}+01$ & $1.53 \mathrm{E}+02$ & $\mathrm{~A}$ & Terrestrial carnivore & 8 & $5.26 \mathrm{E}+02$ \\
\hline 70 & $89347-09-1$ & $3.16 \mathrm{E}+03$ & $3.64 \mathrm{E}+02$ & $8.69 \mathrm{E}+00$ & $\mathrm{~B}$ & Terrestrial carnivore & 12 & $1.08 \mathrm{E}+01$ \\
\hline
\end{tabular}


Table SI - 12. Summary RAIDAR Level III model output for 70 chemicals based on unit emissions to water.

\begin{tabular}{|c|c|c|c|c|c|c|c|}
\hline ID \# & CAS \# & $\begin{array}{c}\mathbf{E}_{\mathrm{A}} \\
\text { t / year }\end{array}$ & $\begin{array}{c}\text { L III-Water } E_{C} \\
t / \text { year }\end{array}$ & $\begin{array}{c}\mathbf{R A F} \\
\mathbf{E}_{\mathbf{A}} / \mathbf{E}_{\mathbf{C}}\end{array}$ & $\begin{array}{c}\text { Risk ID } \\
\text { Bin }\end{array}$ & Media of concern & $\begin{array}{c}\text { Relative } \\
\text { Rank }\end{array}$ \\
\hline 1 & $50-00-0$ & $2.06 \mathrm{E}+03$ & $4.07 \mathrm{E}+06$ & $5.05 \mathrm{E}-04$ & $\mathrm{D}$ & Fish & 37 \\
\hline 2 & $50-32-8$ & $2.41 \mathrm{E}+01$ & $1.27 \mathrm{E}-01$ & $1.90 \mathrm{E}+02$ & $\mathrm{~A}$ & Aquatic mammal & 5 \\
\hline 3 & $51-28-5$ & $3.16 \mathrm{E}+03$ & $4.41 \mathrm{E}+05$ & $7.16 \mathrm{E}-03$ & $\mathrm{D}$ & Avian scavenger & 30 \\
\hline 4 & $67-56-1$ & $2.91 \mathrm{E}+04$ & $1.48 \mathrm{E}+09$ & $1.96 \mathrm{E}-05$ & $\mathrm{E}$ & Plankton & 50 \\
\hline 5 & $67-63-0$ & $2.43 \mathrm{E}+03$ & $2.39 \mathrm{E}+09$ & $1.02 \mathrm{E}-06$ & $\mathrm{E}$ & Fish & 62 \\
\hline 6 & $67-66-3$ & $7.05 \mathrm{E}+01$ & $1.80 \mathrm{E}+08$ & $3.91 \mathrm{E}-07$ & $\mathrm{~F}$ & Fish & 64 \\
\hline 7 & $67-72-1$ & $3.30 \mathrm{E}-02$ & $1.64 \mathrm{E}+06$ & $2.01 \mathrm{E}-08$ & $\mathrm{~F}$ & Fish & 68 \\
\hline 8 & $68-12-2$ & $1.37 \mathrm{E}+01$ & $8.67 \mathrm{E}+08$ & $1.59 \mathrm{E}-08$ & $\mathrm{~F}$ & Avian scavenger & 69 \\
\hline 9 & $70-30-4$ & $3.16 \mathrm{E}+01$ & $9.92 \mathrm{E}-02$ & $3.18 \mathrm{E}+02$ & A & Aquatic mammal & 3 \\
\hline 10 & $71-36-3$ & $9.46 \mathrm{E}+02$ & $1.89 \mathrm{E}+09$ & $5.01 \mathrm{E}-07$ & $\mathrm{~F}$ & Fish & 63 \\
\hline 11 & $71-43-2$ & $1.54 \mathrm{E}+03$ & $9.38 \mathrm{E}+07$ & $1.64 \mathrm{E}-05$ & $\mathrm{E}$ & Fish & 51 \\
\hline 12 & $74-85-1$ & $1.90 \mathrm{E}+03$ & $3.98 \mathrm{E}+08$ & $4.76 \mathrm{E}-06$ & $\mathrm{E}$ & Fish & 55 \\
\hline 13 & $76-60-8$ & $3.16 \mathrm{E}-01$ & $4.01 \mathrm{E}+00$ & $7.88 \mathrm{E}-02$ & $\mathrm{C}$ & Aquatic mammal & 23 \\
\hline 14 & $77-54-3$ & $3.16 \mathrm{E}+01$ & $1.73 \mathrm{E}+03$ & $1.83 \mathrm{E}-02$ & $\mathrm{C}$ & Aquatic mammal & 25 \\
\hline 15 & $78-87-5$ & $3.16 \mathrm{E}+03$ & $1.50 \mathrm{E}+08$ & $2.10 \mathrm{E}-05$ & $\mathrm{E}$ & Fish & 49 \\
\hline 16 & $78-93-3$ & $3.49 \mathrm{E}+03$ & $2.09 \mathrm{E}+09$ & $1.67 \mathrm{E}-06$ & $\mathrm{E}$ & Fish & 59 \\
\hline 17 & 79-01-6 & $7.30 \mathrm{E}+02$ & $5.46 \mathrm{E}+06$ & $1.34 \mathrm{E}-04$ & $\mathrm{D}$ & Fish & 44 \\
\hline 18 & $79-34-5$ & $3.14 \mathrm{E}-01$ & $9.53 \mathrm{E}+07$ & $3.29 \mathrm{E}-09$ & G & Fish & 70 \\
\hline 19 & $79-92-5$ & $3.16 \mathrm{E}+01$ & $1.11 \mathrm{E}+06$ & $2.86 \mathrm{E}-05$ & $\mathrm{E}$ & Fish & 48 \\
\hline 20 & $79-94-7$ & $3.16 \mathrm{E}+03$ & $9.75 \mathrm{E}+00$ & $3.24 \mathrm{E}+02$ & A & Aquatic mammal & 2 \\
\hline 21 & $84-74-2$ & $6.78 \mathrm{E}+00$ & $8.57 \mathrm{E}+04$ & $7.91 \mathrm{E}-05$ & $\mathrm{E}$ & Aquatic mammal & 46 \\
\hline 22 & $85-01-8$ & $1.71 \mathrm{E}+02$ & $5.19 \mathrm{E}+02$ & $3.30 \mathrm{E}-01$ & $\mathrm{C}$ & Aquatic mammal & 17 \\
\hline 23 & $85-68-7$ & $2.09 \mathrm{E}+01$ & $4.09 \mathrm{E}+04$ & $5.11 \mathrm{E}-04$ & $\mathrm{D}$ & Aquatic mammal & 36 \\
\hline
\end{tabular}




\begin{tabular}{|c|c|c|c|c|c|c|c|}
\hline ID \# & CAS \# & $\begin{array}{c}E_{A} \\
t / \text { year }\end{array}$ & $\begin{array}{c}\text { L III-Water } E_{C} \\
\text { t / year }\end{array}$ & $\begin{array}{c}\mathbf{R A F} \\
\mathbf{E}_{\mathbf{A}} / \mathbf{E}_{\mathbf{C}}\end{array}$ & $\begin{array}{c}\text { Risk ID } \\
\text { Bin }\end{array}$ & Media of concern & $\begin{array}{c}\text { Relative } \\
\text { Rank }\end{array}$ \\
\hline 24 & $87-86-5$ & $3.16 \mathrm{E}+03$ & $2.51 \mathrm{E}+02$ & $1.26 \mathrm{E}+01$ & $\mathrm{~B}$ & Aquatic mammal & 7 \\
\hline 25 & $91-20-3$ & $2.78 \mathrm{E}+02$ & $3.65 \mathrm{E}+04$ & $7.63 \mathrm{E}-03$ & $\mathrm{D}$ & Aquatic mammal & 29 \\
\hline 26 & $95-50-1$ & $1.10 \mathrm{E}+01$ & $7.06 \mathrm{E}+06$ & $1.56 \mathrm{E}-06$ & $\mathrm{E}$ & Fish & 60 \\
\hline 27 & $95-63-6$ & $9.85 \mathrm{E}+02$ & $4.67 \mathrm{E}+06$ & $2.11 \mathrm{E}-04$ & $\mathrm{D}$ & Fish & 40 \\
\hline 28 & $101-61-1$ & $3.16 \mathrm{E}-01$ & $2.50 \mathrm{E}+04$ & $1.26 \mathrm{E}-05$ & $\mathrm{E}$ & Aquatic mammal & 52 \\
\hline 29 & $104-40-5$ & $1.93 \mathrm{E}+02$ & $8.67 \mathrm{E}+00$ & $2.22 \mathrm{E}+01$ & $\mathrm{~B}$ & Aquatic mammal & 6 \\
\hline 30 & $106-46-7$ & $1.60 \mathrm{E}+01$ & $6.75 E+06$ & $2.37 \mathrm{E}-06$ & $\mathrm{E}$ & Fish & 56 \\
\hline 31 & $107-06-2$ & $9.52 \mathrm{E}+00$ & $3.87 \mathrm{E}+08$ & $2.46 \mathrm{E}-08$ & $\mathrm{~F}$ & Fish & 67 \\
\hline 32 & $107-21-1$ & $3.64 \mathrm{E}+03$ & $1.84 \mathrm{E}+09$ & $1.98 \mathrm{E}-06$ & $\mathrm{E}$ & Avian scavenger & 58 \\
\hline 33 & $108-10-1$ & $7.52 \mathrm{E}+02$ & $6.54 \mathrm{E}+08$ & $1.15 \mathrm{E}-06$ & $\mathrm{E}$ & Fish & 61 \\
\hline 34 & $108-88-3$ & $7.27 \mathrm{E}+03$ & $3.55 \mathrm{E}+07$ & $2.05 \mathrm{E}-04$ & $\mathrm{D}$ & Fish & 41 \\
\hline 35 & $108-90-7$ & $4.56 \mathrm{E}+00$ & $2.41 \mathrm{E}+07$ & $1.90 \mathrm{E}-07$ & $\mathrm{~F}$ & Fish & 65 \\
\hline 36 & $110-54-3$ & $6.04 \mathrm{E}+03$ & $3.30 \mathrm{E}+06$ & $1.83 \mathrm{E}-03$ & $\mathrm{D}$ & Fish & 33 \\
\hline 37 & $110-82-7$ & $1.64 \mathrm{E}+03$ & $6.08 \mathrm{E}+06$ & $2.71 \mathrm{E}-04$ & $\mathrm{D}$ & Fish & 39 \\
\hline 38 & $111-42-2$ & $3.55 \mathrm{E}+02$ & $1.61 \mathrm{E}+08$ & $2.20 \mathrm{E}-06$ & $\mathrm{E}$ & Avian scavenger & 57 \\
\hline 39 & $115-07-1$ & $1.11 \mathrm{E}+03$ & $1.56 \mathrm{E}+08$ & $7.15 \mathrm{E}-06$ & $\mathrm{E}$ & Fish & 53 \\
\hline 40 & $117-81-7$ & $8.02 \mathrm{E}+01$ & $4.53 \mathrm{E}+02$ & $1.77 \mathrm{E}-01$ & $\mathrm{C}$ & Aquatic mammal & 20 \\
\hline 41 & $118-74-1$ & $2.95 \mathrm{E}-02$ & $6.99 \mathrm{E}+02$ & $4.22 \mathrm{E}-05$ & E & Aquatic mammal & 47 \\
\hline 42 & $120-12-7$ & $1.22 \mathrm{E}+01$ & $3.50 \mathrm{E}+00$ & $3.48 \mathrm{E}+00$ & $\mathrm{~B}$ & Aquatic mammal & 13 \\
\hline 43 & $120-83-2$ & $3.16 \mathrm{E}+01$ & $4.48 \mathrm{E}+03$ & $7.05 \mathrm{E}-03$ & $\mathrm{D}$ & Aquatic mammal & 31 \\
\hline 44 & $124-18-5$ & $3.16 \mathrm{E}+01$ & $2.02 \mathrm{E}+05$ & $1.56 \mathrm{E}-04$ & $\mathrm{D}$ & Fish & 42 \\
\hline 45 & $127-18-4$ & $4.21 \mathrm{E}+01$ & $8.37 \mathrm{E}+06$ & 5.04E-06 & $\mathrm{E}$ & Fish & 54 \\
\hline 46 & $129-00-0$ & $9.37 \mathrm{E}+01$ & $9.51 \mathrm{E}-02$ & $9.86 \mathrm{E}+02$ & A & Aquatic mammal & 1 \\
\hline 47 & $603-35-0$ & $3.16 \mathrm{E}+01$ & $5.00 \mathrm{E}+02$ & $6.32 \mathrm{E}-02$ & $\mathrm{C}$ & Aquatic mammal & 24 \\
\hline
\end{tabular}




\begin{tabular}{|c|c|c|c|c|c|c|c|}
\hline ID \# & CAS \# & $\begin{array}{c}E_{A} \\
t / \text { year }\end{array}$ & $\begin{array}{c}\text { L III-Water } E_{C} \\
t / \text { year }\end{array}$ & $\begin{array}{c}\mathbf{R A F} \\
\mathbf{E}_{\mathbf{A}} / \mathbf{E}_{\mathrm{C}}\end{array}$ & $\begin{array}{c}\text { Risk ID } \\
\text { Bin }\end{array}$ & Media of concern & $\begin{array}{c}\text { Relative } \\
\text { Rank }\end{array}$ \\
\hline 48 & $608-93-5$ & $3.16 \mathrm{E}-01$ & $2.26 \mathrm{E}+03$ & $1.40 \mathrm{E}-04$ & $\mathrm{D}$ & Aquatic mammal & 43 \\
\hline 49 & $1163-19-5$ & $5.77 \mathrm{E}+00$ & $3.77 \mathrm{E}+02$ & $1.53 \mathrm{E}-02$ & $\mathrm{C}$ & Aquatic mammal & 27 \\
\hline 50 & $1222-05-5$ & $3.16 \mathrm{E}+01$ & $1.10 \mathrm{E}+02$ & $2.87 \mathrm{E}-01$ & $\mathrm{C}$ & Aquatic mammal & 18 \\
\hline 51 & $1330-78-5$ & $3.16 \mathrm{E}+03$ & $3.43 \mathrm{E}+02$ & $9.21 \mathrm{E}+00$ & $\mathrm{~B}$ & Aquatic mammal & 12 \\
\hline 52 & $1582-09-8$ & $3.16 \mathrm{E}-01$ & $2.31 \mathrm{E}+01$ & $1.37 \mathrm{E}-02$ & $\mathrm{C}$ & Aquatic mammal & 28 \\
\hline 53 & $1897-45-6$ & $3.16 \mathrm{E}+01$ & $2.56 \mathrm{E}+01$ & $1.23 \mathrm{E}+00$ & $\mathrm{~B}$ & Aquatic mammal & 14 \\
\hline 54 & $1912-24-9$ & $3.16 \mathrm{E}+01$ & $1.72 \mathrm{E}+04$ & $1.83 \mathrm{E}-03$ & $\mathrm{D}$ & Avian scavenger & 32 \\
\hline 55 & $3380-34-5$ & $3.16 \mathrm{E}+01$ & $1.48 \mathrm{E}-01$ & $2.14 \mathrm{E}+02$ & A & Aquatic mammal & 4 \\
\hline 56 & $3846-71-7$ & $3.16 \mathrm{E}+01$ & $1.17 \mathrm{E}+02$ & $2.69 \mathrm{E}-01$ & $\mathrm{C}$ & Aquatic mammal & 19 \\
\hline 57 & $4979-32-2$ & $3.16 \mathrm{E}+01$ & $2.74 \mathrm{E}+05$ & $1.15 \mathrm{E}-04$ & $\mathrm{D}$ & Aquatic mammal & 45 \\
\hline 58 & $5285-60-9$ & $3.16 \mathrm{E}+03$ & $3.04 \mathrm{E}+02$ & $1.04 \mathrm{E}+01$ & $\mathrm{~B}$ & Aquatic mammal & 10 \\
\hline 59 & $6386-38-5$ & $3.16 \mathrm{E}+01$ & $1.78 \mathrm{E}+03$ & $1.77 \mathrm{E}-02$ & $\mathrm{C}$ & Aquatic mammal & 26 \\
\hline 60 & $13560-89-9$ & $3.16 \mathrm{E}+01$ & $2.28 \mathrm{E}+02$ & $1.39 \mathrm{E}-01$ & $\mathrm{C}$ & Aquatic mammal & 22 \\
\hline 61 & $15646-96-5$ & $3.16 \mathrm{E}+01$ & $7.42 \mathrm{E}+04$ & $4.26 \mathrm{E}-04$ & $\mathrm{D}$ & Aquatic mammal & 38 \\
\hline 62 & $23593-75-1$ & $3.16 \mathrm{E}-01$ & $3.85 \mathrm{E}+02$ & $8.21 \mathrm{E}-04$ & $\mathrm{D}$ & Aquatic mammal & 35 \\
\hline 63 & $25973-55-1$ & $3.16 \mathrm{E}+01$ & $9.01 \mathrm{E}+01$ & $3.51 \mathrm{E}-01$ & $\mathrm{C}$ & Aquatic mammal & 16 \\
\hline 64 & $26140-60-3$ & $3.16 \mathrm{E}+01$ & $1.99 \mathrm{E}+02$ & $1.59 \mathrm{E}-01$ & $\mathrm{C}$ & Aquatic mammal & 21 \\
\hline 65 & $32534-81-9$ & $3.16 \mathrm{E}+01$ & $8.29 \mathrm{E}+01$ & $3.81 \mathrm{E}-01$ & $\mathrm{C}$ & Aquatic mammal & 15 \\
\hline 66 & $32536-52-0$ & $3.16 \mathrm{E}+03$ & $2.80 \mathrm{E}+02$ & $1.13 \mathrm{E}+01$ & $\mathrm{~B}$ & Aquatic mammal & 9 \\
\hline 67 & $54079-53-7$ & $3.16 \mathrm{E}+01$ & $2.13 \mathrm{E}+04$ & $1.48 \mathrm{E}-03$ & $\mathrm{D}$ & Aquatic mammal & 34 \\
\hline 68 & $54827-17-7$ & $3.16 \mathrm{E}-01$ & $5.03 \mathrm{E}+06$ & $6.28 \mathrm{E}-08$ & $\mathrm{~F}$ & Aquatic mammal & 66 \\
\hline 69 & 63936-56-1 & $3.16 \mathrm{E}+03$ & $3.07 \mathrm{E}+02$ & $1.03 \mathrm{E}+01$ & $\mathrm{~B}$ & Aquatic mammal & 11 \\
\hline 70 & $89347-09-1$ & $3.16 \mathrm{E}+03$ & $2.65 \mathrm{E}+02$ & $1.19 \mathrm{E}+01$ & $\mathrm{~B}$ & Aquatic mammal & 8 \\
\hline
\end{tabular}


Table SI - 13. Summary RAIDAR Level III model output for 70 chemicals based on unit emissions to soil.

\begin{tabular}{|c|c|c|c|c|c|c|c|}
\hline ID \# & CAS \# & $\begin{array}{c}\mathrm{E}_{\mathrm{A}} \\
\mathrm{t} / \text { year }\end{array}$ & $\begin{array}{c}\text { L III-Soil E } \\
\text { t / year }\end{array}$ & $\begin{array}{c}\mathbf{R A F} \\
\mathbf{E}_{\mathbf{A}} / \mathbf{E}_{\mathbf{C}}\end{array}$ & $\begin{array}{c}\text { Risk ID } \\
\text { Bin }\end{array}$ & Media of concern & $\begin{array}{c}\text { Relative } \\
\text { Rank }\end{array}$ \\
\hline 1 & $50-00-0$ & $2.06 \mathrm{E}+03$ & $2.02 \mathrm{E}+05$ & $1.02 \mathrm{E}-02$ & $\mathrm{C}$ & Terrestrial invertebrate & 16 \\
\hline 2 & $50-32-8$ & $2.41 \mathrm{E}+01$ & $3.77 \mathrm{E}+01$ & 6.39E-01 & $\mathrm{C}$ & Terrestrial carnivore & 5 \\
\hline 3 & $51-28-5$ & $3.16 \mathrm{E}+03$ & $8.21 \mathrm{E}+04$ & $3.85 \mathrm{E}-02$ & $\mathrm{C}$ & Avian & 14 \\
\hline 4 & $67-56-1$ & $2.91 \mathrm{E}+04$ & $3.00 \mathrm{E}+07$ & $9.70 \mathrm{E}-04$ & $\mathrm{D}$ & Root vegetation & 24 \\
\hline 5 & $67-63-0$ & $2.43 \mathrm{E}+03$ & $5.24 \mathrm{E}+07$ & 4.64E-05 & $\mathrm{E}$ & Terrestrial invertebrate & 39 \\
\hline 6 & $67-66-3$ & $7.05 \mathrm{E}+01$ & $1.87 \mathrm{E}+09$ & $3.76 \mathrm{E}-08$ & $\mathrm{~F}$ & Terrestrial invertebrate & 67 \\
\hline 7 & $67-72-1$ & $3.30 \mathrm{E}-02$ & $2.29 \mathrm{E}+07$ & $1.44 \mathrm{E}-09$ & $\mathrm{G}$ & Terrestrial invertebrate & 70 \\
\hline 8 & $68-12-2$ & $1.37 \mathrm{E}+01$ & $4.98 \mathrm{E}+07$ & $2.76 \mathrm{E}-07$ & $\mathrm{~F}$ & Root vegetation & 62 \\
\hline 9 & $70-30-4$ & $3.16 \mathrm{E}+01$ & $5.62 \mathrm{E}+01$ & $5.62 \mathrm{E}-01$ & $\mathrm{C}$ & Terrestrial carnivore & 6 \\
\hline 10 & $71-36-3$ & $9.46 \mathrm{E}+02$ & $8.42 \mathrm{E}+07$ & $1.12 \mathrm{E}-05$ & $\mathrm{E}$ & Terrestrial invertebrate & 45 \\
\hline 11 & $71-43-2$ & $1.54 \mathrm{E}+03$ & $3.19 \mathrm{E}+08$ & $4.82 \mathrm{E}-06$ & $\mathrm{E}$ & Terrestrial invertebrate & 53 \\
\hline 12 & $74-85-1$ & $1.90 \mathrm{E}+03$ & $6.71 \mathrm{E}+08$ & $2.83 \mathrm{E}-06$ & $\mathrm{E}$ & Terrestrial invertebrate & 54 \\
\hline 13 & $76-60-8$ & $3.16 \mathrm{E}-01$ & $2.12 \mathrm{E}+03$ & $1.49 \mathrm{E}-04$ & $\mathrm{D}$ & Terrestrial carnivore & 33 \\
\hline 14 & $77-54-3$ & $3.16 \mathrm{E}+01$ & $1.61 \mathrm{E}+05$ & $1.96 \mathrm{E}-04$ & $\mathrm{D}$ & Terrestrial carnivore & 30 \\
\hline 15 & $78-87-5$ & $3.16 \mathrm{E}+03$ & $3.80 \mathrm{E}+08$ & $8.31 \mathrm{E}-06$ & $\mathrm{E}$ & Terrestrial invertebrate & 47 \\
\hline 16 & $78-93-3$ & $3.49 \mathrm{E}+03$ & $7.99 \mathrm{E}+07$ & 4.37E-05 & E & Terrestrial invertebrate & 41 \\
\hline 17 & $79-01-6$ & $7.30 \mathrm{E}+02$ & $7.31 \mathrm{E}+07$ & $9.98 \mathrm{E}-06$ & $\mathrm{E}$ & Terrestrial invertebrate & 46 \\
\hline 18 & $79-34-5$ & $3.14 \mathrm{E}-01$ & $1.67 \mathrm{E}+08$ & $1.88 \mathrm{E}-09$ & $\mathrm{G}$ & Terrestrial invertebrate & 69 \\
\hline 19 & $79-92-5$ & $3.16 \mathrm{E}+01$ & $7.66 \mathrm{E}+07$ & 4.12E-07 & $\mathrm{F}$ & Terrestrial invertebrate & 60 \\
\hline 20 & 79-94-7 & $3.16 \mathrm{E}+03$ & $6.06 \mathrm{E}+03$ & $5.22 \mathrm{E}-01$ & $\mathrm{C}$ & Terrestrial carnivore & 7 \\
\hline 21 & $84-74-2$ & $6.78 \mathrm{E}+00$ & $1.08 \mathrm{E}+06$ & $6.30 \mathrm{E}-06$ & $\mathrm{E}$ & Terrestrial carnivore & 49 \\
\hline 22 & $85-01-8$ & $1.71 \mathrm{E}+02$ & $5.99 \mathrm{E}+03$ & $2.86 \mathrm{E}-02$ & $\mathrm{C}$ & Terrestrial carnivore & 15 \\
\hline 23 & $85-68-7$ & $2.09 \mathrm{E}+01$ & $9.88 \mathrm{E}+05$ & $2.11 \mathrm{E}-05$ & $\mathrm{E}$ & Terrestrial carnivore & 44 \\
\hline
\end{tabular}




\begin{tabular}{|c|c|c|c|c|c|c|c|}
\hline ID \# & CAS \# & $\begin{array}{c}\mathbf{E}_{\mathrm{A}} \\
\mathrm{t} / \text { year }\end{array}$ & $\begin{array}{c}\text { L III-Soil E } \\
\text { t / year }\end{array}$ & $\begin{array}{c}\mathbf{R A F} \\
\mathbf{E}_{\mathbf{A}} / \mathbf{E}_{\mathbf{C}}\end{array}$ & $\begin{array}{c}\text { Risk ID } \\
\text { Bin }\end{array}$ & Media of concern & $\begin{array}{c}\text { Relative } \\
\text { Rank }\end{array}$ \\
\hline 24 & $87-86-5$ & $3.16 \mathrm{E}+03$ & $2.50 \mathrm{E}+02$ & $1.26 \mathrm{E}+01$ & $\mathrm{~B}$ & Terrestrial carnivore & 2 \\
\hline 25 & $91-20-3$ & $2.78 \mathrm{E}+02$ & $5.57 \mathrm{E}+04$ & $5.00 \mathrm{E}-03$ & $\mathrm{D}$ & Human & 20 \\
\hline 26 & $95-50-1$ & $1.10 \mathrm{E}+01$ & $3.25 \mathrm{E}+07$ & $3.39 \mathrm{E}-07$ & $\mathrm{~F}$ & Terrestrial invertebrate & 61 \\
\hline 27 & $95-63-6$ & $9.85 \mathrm{E}+02$ & $3.59 \mathrm{E}+07$ & $2.75 \mathrm{E}-05$ & $\mathrm{E}$ & Terrestrial invertebrate & 43 \\
\hline 28 & $101-61-1$ & $3.16 \mathrm{E}-01$ & $6.01 \mathrm{E}+04$ & $5.26 \mathrm{E}-06$ & $\mathrm{E}$ & Terrestrial carnivore & 52 \\
\hline 29 & $104-40-5$ & $1.93 \mathrm{E}+02$ & $3.90 \mathrm{E}+03$ & $4.94 \mathrm{E}-02$ & $\mathrm{C}$ & Terrestrial carnivore & 12 \\
\hline 30 & $106-46-7$ & $1.60 \mathrm{E}+01$ & $2.70 \mathrm{E}+07$ & $5.92 \mathrm{E}-07$ & $\mathrm{~F}$ & Terrestrial invertebrate & 58 \\
\hline 31 & $107-06-2$ & $9.52 \mathrm{E}+00$ & $2.73 \mathrm{E}+08$ & $3.49 \mathrm{E}-08$ & $\mathrm{~F}$ & Terrestrial invertebrate & 68 \\
\hline 32 & $107-21-1$ & $3.64 \mathrm{E}+03$ & $8.28 \mathrm{E}+07$ & $4.39 \mathrm{E}-05$ & $\mathrm{E}$ & Root vegetation & 40 \\
\hline 33 & $108-10-1$ & $7.52 \mathrm{E}+02$ & $1.21 \mathrm{E}+08$ & $6.22 \mathrm{E}-06$ & E & Terrestrial invertebrate & 50 \\
\hline 34 & $108-88-3$ & $7.27 \mathrm{E}+03$ & $1.56 \mathrm{E}+08$ & $4.65 \mathrm{E}-05$ & E & Terrestrial invertebrate & 38 \\
\hline 35 & $108-90-7$ & $4.56 \mathrm{E}+00$ & $1.05 \mathrm{E}+08$ & 4.34E-08 & $\mathrm{F}$ & Terrestrial invertebrate & 66 \\
\hline 36 & $110-54-3$ & $6.04 \mathrm{E}+03$ & $2.64 \mathrm{E}+09$ & $2.28 \mathrm{E}-06$ & E & Terrestrial invertebrate & 55 \\
\hline 37 & $110-82-7$ & $1.64 \mathrm{E}+03$ & $1.02 \mathrm{E}+09$ & $1.61 \mathrm{E}-06$ & $\mathrm{E}$ & Terrestrial invertebrate & 57 \\
\hline 38 & $111-42-2$ & $3.55 \mathrm{E}+02$ & $7.29 \mathrm{E}+06$ & $4.87 \mathrm{E}-05$ & $\mathrm{E}$ & Root vegetation & 37 \\
\hline 39 & $115-07-1$ & $1.11 \mathrm{E}+03$ & $2.21 \mathrm{E}+09$ & $5.04 \mathrm{E}-07$ & $\mathrm{~F}$ & Terrestrial invertebrate & 59 \\
\hline 40 & $117-81-7$ & $8.02 \mathrm{E}+01$ & $6.20 \mathrm{E}+05$ & $1.29 \mathrm{E}-04$ & $\mathrm{D}$ & Terrestrial carnivore & 34 \\
\hline 41 & $118-74-1$ & $2.95 \mathrm{E}-02$ & $1.56 \mathrm{E}+04$ & $1.88 \mathrm{E}-06$ & $\mathrm{E}$ & Terrestrial carnivore & 56 \\
\hline 42 & $120-12-7$ & $1.22 \mathrm{E}+01$ & $5.28 \mathrm{E}+01$ & $2.30 \mathrm{E}-01$ & $\mathrm{C}$ & Terrestrial carnivore & 8 \\
\hline 43 & $120-83-2$ & $3.16 \mathrm{E}+01$ & $6.18 \mathrm{E}+03$ & $5.11 \mathrm{E}-03$ & $\mathrm{D}$ & Human & 19 \\
\hline 44 & $124-18-5$ & $3.16 \mathrm{E}+01$ & $1.41 \mathrm{E}+08$ & $2.24 \mathrm{E}-07$ & $\mathrm{~F}$ & Terrestrial invertebrate & 63 \\
\hline 45 & $127-18-4$ & $4.21 \mathrm{E}+01$ & $4.40 \mathrm{E}+08$ & $9.57 \mathrm{E}-08$ & $\mathrm{~F}$ & Terrestrial invertebrate & 64 \\
\hline 46 & $129-00-0$ & $9.37 \mathrm{E}+01$ & $3.55 \mathrm{E}+00$ & $2.64 \mathrm{E}+01$ & $\mathrm{~B}$ & Terrestrial carnivore & 1 \\
\hline 47 & $603-35-0$ & $3.16 \mathrm{E}+01$ & $2.03 \mathrm{E}+05$ & $1.55 \mathrm{E}-04$ & $\mathrm{D}$ & Terrestrial carnivore & 32 \\
\hline
\end{tabular}




\begin{tabular}{|c|c|c|c|c|c|c|c|}
\hline ID \# & CAS \# & $\begin{array}{c}\mathbf{E}_{\mathrm{A}} \\
\mathrm{t} / \text { year }\end{array}$ & $\begin{array}{c}\text { L III-Soil } E_{C} \\
\text { t / year }\end{array}$ & $\begin{array}{c}\mathbf{R A F} \\
\mathbf{E}_{\mathbf{A}} / \mathbf{E}_{\mathbf{C}}\end{array}$ & $\begin{array}{c}\text { Risk ID } \\
\text { Bin }\end{array}$ & Media of concern & $\begin{array}{c}\text { Relative } \\
\text { Rank }\end{array}$ \\
\hline 48 & $608-93-5$ & $3.16 \mathrm{E}-01$ & $4.81 \mathrm{E}+04$ & $6.57 \mathrm{E}-06$ & E & Terrestrial carnivore & 48 \\
\hline 49 & $1163-19-5$ & $5.77 \mathrm{E}+00$ & $4.87 \mathrm{E}+04$ & $1.19 \mathrm{E}-04$ & $\mathrm{D}$ & Terrestrial carnivore & 35 \\
\hline 50 & $1222-05-5$ & $3.16 \mathrm{E}+01$ & $4.15 \mathrm{E}+04$ & $7.62 \mathrm{E}-04$ & $\mathrm{D}$ & Terrestrial carnivore & 25 \\
\hline 51 & $1330-78-5$ & $3.16 \mathrm{E}+03$ & $3.42 \mathrm{E}+05$ & $9.24 \mathrm{E}-03$ & $\mathrm{D}$ & Terrestrial carnivore & 17 \\
\hline 52 & $1582-09-8$ & $3.16 \mathrm{E}-01$ & $1.11 \mathrm{E}+03$ & 2.84E-04 & $\mathrm{D}$ & Terrestrial carnivore & 28 \\
\hline 53 & $1897-45-6$ & $3.16 \mathrm{E}+01$ & $2.50 \mathrm{E}+01$ & $1.26 \mathrm{E}+00$ & B & Terrestrial carnivore & 4 \\
\hline 54 & $1912-24-9$ & $3.16 \mathrm{E}+01$ & $4.77 \mathrm{E}+03$ & $6.62 \mathrm{E}-03$ & $\mathrm{D}$ & Avian & 18 \\
\hline 55 & $3380-34-5$ & $3.16 \mathrm{E}+01$ & $5.57 \mathrm{E}+00$ & $5.68 \mathrm{E}+00$ & $\mathrm{~B}$ & Terrestrial carnivore & 3 \\
\hline 56 & $3846-71-7$ & $3.16 \mathrm{E}+01$ & $7.12 \mathrm{E}+04$ & 4.44E-04 & $\mathrm{D}$ & Terrestrial carnivore & 27 \\
\hline 57 & $4979-32-2$ & $3.16 \mathrm{E}+01$ & $3.59 \mathrm{E}+05$ & $8.81 \mathrm{E}-05$ & E & Terrestrial carnivore & 36 \\
\hline 58 & $5285-60-9$ & $3.16 \mathrm{E}+03$ & $8.03 E+04$ & $3.94 \mathrm{E}-02$ & $\mathrm{C}$ & Terrestrial carnivore & 13 \\
\hline 59 & $6386-38-5$ & $3.16 \mathrm{E}+01$ & $1.64 \mathrm{E}+05$ & $1.93 \mathrm{E}-04$ & $\mathrm{D}$ & Terrestrial carnivore & 31 \\
\hline 60 & $13560-89-9$ & $3.16 \mathrm{E}+01$ & $2.08 \mathrm{E}+04$ & $1.52 \mathrm{E}-03$ & $\mathrm{D}$ & Terrestrial carnivore & 22 \\
\hline 61 & $15646-96-5$ & $3.16 \mathrm{E}+01$ & $1.12 \mathrm{E}+06$ & $2.82 \mathrm{E}-05$ & $\mathrm{E}$ & Terrestrial carnivore & 42 \\
\hline 62 & $23593-75-1$ & $3.16 \mathrm{E}-01$ & $5.81 \mathrm{E}+04$ & $5.43 \mathrm{E}-06$ & $\mathrm{E}$ & Terrestrial carnivore & 51 \\
\hline 63 & $25973-55-1$ & $3.16 \mathrm{E}+01$ & $6.54 \mathrm{E}+04$ & $4.83 \mathrm{E}-04$ & $\mathrm{D}$ & Terrestrial carnivore & 26 \\
\hline 64 & $26140-60-3$ & $3.16 \mathrm{E}+01$ & $1.29 \mathrm{E}+05$ & $2.45 \mathrm{E}-04$ & $\mathrm{D}$ & Terrestrial carnivore & 29 \\
\hline 65 & $32534-81-9$ & $3.16 \mathrm{E}+01$ & $2.39 \mathrm{E}+04$ & $1.32 \mathrm{E}-03$ & $\mathrm{D}$ & Aquatic mammal & 23 \\
\hline 66 & $32536-52-0$ & $3.16 \mathrm{E}+03$ & $2.55 \mathrm{E}+04$ & $1.24 \mathrm{E}-01$ & $\mathrm{C}$ & Terrestrial carnivore & 9 \\
\hline 67 & $54079-53-7$ & $3.16 \mathrm{E}+01$ & $1.80 \mathrm{E}+04$ & $1.75 \mathrm{E}-03$ & $\mathrm{D}$ & Avian scavenger & 21 \\
\hline 68 & $54827-17-7$ & $3.16 \mathrm{E}-01$ & $3.47 \mathrm{E}+06$ & $9.11 \mathrm{E}-08$ & $\mathrm{~F}$ & Avian scavenger & 65 \\
\hline 69 & $63936-56-1$ & $3.16 \mathrm{E}+03$ & $2.80 \mathrm{E}+04$ & $1.13 \mathrm{E}-01$ & $\mathrm{C}$ & Terrestrial carnivore & 10 \\
\hline 70 & $89347-09-1$ & $3.16 \mathrm{E}+03$ & $5.91 \mathrm{E}+04$ & $5.34 \mathrm{E}-02$ & $\mathrm{C}$ & Terrestrial carnivore & 11 \\
\hline
\end{tabular}


Table SI - 14. Summary RAIDAR Level III model output for 70 chemicals based on equal unit emissions to air, water and soil.

\begin{tabular}{|c|c|c|c|c|c|c|c|}
\hline ID \# & CAS \# & $\begin{array}{c}\mathbf{E}_{\mathrm{A}} \\
\mathrm{t} / \text { year }\end{array}$ & $\begin{array}{c}\text { L III-AWS E } \\
\text { t / year }\end{array}$ & $\begin{array}{c}\mathbf{R A F} \\
\mathbf{E}_{\mathbf{A}} / \mathbf{E}_{\mathbf{C}}\end{array}$ & $\begin{array}{c}\text { Risk ID } \\
\text { Bin }\end{array}$ & Media of concern & $\begin{array}{c}\text { Relative } \\
\text { Rank }\end{array}$ \\
\hline 1 & $50-00-0$ & $2.06 \mathrm{E}+03$ & $6.05 \mathrm{E}+05$ & $3.41 \mathrm{E}-03$ & $\mathrm{D}$ & Terrestrial invertebrate & 35 \\
\hline 2 & $50-32-8$ & $2.41 \mathrm{E}+01$ & $3.75 \mathrm{E}-01$ & $6.41 \mathrm{E}+01$ & $\mathrm{~B}$ & Aquatic mammal & 8 \\
\hline 3 & $51-28-5$ & $3.16 \mathrm{E}+03$ & $1.26 \mathrm{E}+01$ & $2.51 \mathrm{E}+02$ & A & Foliage vegetation & 5 \\
\hline 4 & $67-56-1$ & $2.91 \mathrm{E}+04$ & $8.66 \mathrm{E}+07$ & $3.35 \mathrm{E}-04$ & $\mathrm{D}$ & Root vegetation & 42 \\
\hline 5 & $67-63-0$ & $2.43 \mathrm{E}+03$ & $1.56 \mathrm{E}+08$ & $1.56 \mathrm{E}-05$ & $E$ & Terrestrial invertebrate & 52 \\
\hline 6 & $67-66-3$ & $7.05 \mathrm{E}+01$ & $5.38 \mathrm{E}+08$ & $1.31 \mathrm{E}-07$ & $\mathrm{~F}$ & Fish & 66 \\
\hline 7 & $67-72-1$ & $3.30 \mathrm{E}-02$ & $4.88 \mathrm{E}+06$ & $6.76 \mathrm{E}-09$ & G & Fish & 69 \\
\hline 8 & $68-12-2$ & $1.37 \mathrm{E}+01$ & $4.71 \mathrm{E}+01$ & $2.92 \mathrm{E}-01$ & $\mathrm{C}$ & Foliage vegetation & 20 \\
\hline 9 & $70-30-4$ & $3.16 \mathrm{E}+01$ & $3.57 \mathrm{E}-02$ & $8.85 \mathrm{E}+02$ & $\mathrm{~A}$ & Terrestrial carnivore & 3 \\
\hline 10 & $71-36-3$ & $9.46 \mathrm{E}+02$ & $2.50 \mathrm{E}+08$ & $3.78 \mathrm{E}-06$ & E & Terrestrial invertebrate & 59 \\
\hline 11 & $71-43-2$ & $1.54 \mathrm{E}+03$ & $2.77 \mathrm{E}+08$ & $5.54 \mathrm{E}-06$ & E & Fish & 58 \\
\hline 12 & $74-85-1$ & $1.90 \mathrm{E}+03$ & $1.18 \mathrm{E}+09$ & $1.60 \mathrm{E}-06$ & $\mathrm{E}$ & Fish & 63 \\
\hline 13 & $76-60-8$ & $3.16 \mathrm{E}-01$ & $1.59 \mathrm{E}+00$ & $1.99 \mathrm{E}-01$ & $\mathrm{C}$ & Terrestrial carnivore & 21 \\
\hline 14 & $77-54-3$ & $3.16 \mathrm{E}+01$ & $5.16 \mathrm{E}+03$ & $6.13 \mathrm{E}-03$ & $\mathrm{D}$ & Aquatic mammal & 33 \\
\hline 15 & $78-87-5$ & $3.16 \mathrm{E}+03$ & $4.40 \mathrm{E}+08$ & 7.19E-06 & E & Fish & 56 \\
\hline 16 & $78-93-3$ & $3.49 \mathrm{E}+03$ & $2.39 \mathrm{E}+08$ & $1.46 \mathrm{E}-05$ & $\mathrm{E}$ & Terrestrial invertebrate & 53 \\
\hline 17 & $79-01-6$ & $7.30 \mathrm{E}+02$ & $1.63 \mathrm{E}+07$ & $4.48 \mathrm{E}-05$ & $\mathrm{E}$ & Fish & 50 \\
\hline 18 & $79-34-5$ & $3.14 \mathrm{E}-01$ & $2.75 \mathrm{E}+08$ & $1.14 \mathrm{E}-09$ & G & Fish & 70 \\
\hline 19 & $79-92-5$ & $3.16 \mathrm{E}+01$ & $3.31 \mathrm{E}+06$ & $9.54 \mathrm{E}-06$ & $\mathrm{E}$ & Fish & 55 \\
\hline 20 & $79-94-7$ & $3.16 \mathrm{E}+03$ & $2.07 \mathrm{E}+00$ & $1.53 \mathrm{E}+03$ & A & Terrestrial carnivore & 2 \\
\hline 21 & $84-74-2$ & $6.78 \mathrm{E}+00$ & $7.69 \mathrm{E}+03$ & 8.81E-04 & $\mathrm{D}$ & Terrestrial carnivore & 39 \\
\hline 22 & $85-01-8$ & $1.71 \mathrm{E}+02$ & $1.53 \mathrm{E}+03$ & $1.12 \mathrm{E}-01$ & $\mathrm{C}$ & Aquatic mammal & 25 \\
\hline 23 & $85-68-7$ & $2.09 \mathrm{E}+01$ & $2.81 \mathrm{E}+04$ & $7.42 \mathrm{E}-04$ & $\mathrm{D}$ & Terrestrial carnivore & 40 \\
\hline
\end{tabular}




\begin{tabular}{|c|c|c|c|c|c|c|c|}
\hline ID \# & CAS \# & $\begin{array}{c}\mathbf{E}_{\mathrm{A}} \\
\mathrm{t} / \text { year }\end{array}$ & $\begin{array}{c}\text { L III-AWS E } \\
\text { t / year }\end{array}$ & $\begin{array}{c}\mathbf{R A F} \\
\mathbf{E}_{\mathbf{A}} / \mathbf{E}_{\mathbf{C}}\end{array}$ & $\begin{array}{c}\text { Risk ID } \\
\text { Bin }\end{array}$ & Media of concern & $\begin{array}{c}\text { Relative } \\
\text { Rank }\end{array}$ \\
\hline 24 & $87-86-5$ & $3.16 \mathrm{E}+03$ & $8.03 \mathrm{E}+01$ & $3.93 \mathrm{E}+01$ & $\mathrm{~B}$ & Terrestrial carnivore & 11 \\
\hline 25 & $91-20-3$ & $2.78 \mathrm{E}+02$ & $1.05 \mathrm{E}+05$ & $2.66 \mathrm{E}-03$ & $\mathrm{D}$ & Aquatic mammal & 36 \\
\hline 26 & $95-50-1$ & $1.10 \mathrm{E}+01$ & $2.08 \mathrm{E}+07$ & $5.29 \mathrm{E}-07$ & $\mathrm{~F}$ & Fish & 65 \\
\hline 27 & $95-63-6$ & $9.85 \mathrm{E}+02$ & $1.39 \mathrm{E}+07$ & $7.09 \mathrm{E}-05$ & E & Fish & 46 \\
\hline 28 & $101-61-1$ & $3.16 \mathrm{E}-01$ & $5.11 \mathrm{E}+04$ & $6.18 \mathrm{E}-06$ & $\mathrm{E}$ & Terrestrial carnivore & 57 \\
\hline 29 & $104-40-5$ & $1.93 \mathrm{E}+02$ & $2.59 \mathrm{E}+01$ & $7.43 \mathrm{E}+00$ & $\mathrm{~B}$ & Aquatic mammal & 12 \\
\hline 30 & $106-46-7$ & $1.60 \mathrm{E}+01$ & $1.98 \mathrm{E}+07$ & $8.08 \mathrm{E}-07$ & $\mathrm{~F}$ & Fish & 64 \\
\hline 31 & $107-06-2$ & $9.52 \mathrm{E}+00$ & $8.16 \mathrm{E}+08$ & $1.17 \mathrm{E}-08$ & $\mathrm{~F}$ & Terrestrial invertebrate & 68 \\
\hline 32 & $107-21-1$ & $3.64 \mathrm{E}+03$ & $1.79 \mathrm{E}+07$ & $2.03 \mathrm{E}-04$ & $\mathrm{D}$ & Foliage vegetation & 43 \\
\hline 33 & $108-10-1$ & $7.52 \mathrm{E}+02$ & $3.62 \mathrm{E}+08$ & $2.08 \mathrm{E}-06$ & E & Terrestrial invertebrate & 61 \\
\hline 34 & $108-88-3$ & $7.27 \mathrm{E}+03$ & $1.05 \mathrm{E}+08$ & $6.90 \mathrm{E}-05$ & E & Fish & 47 \\
\hline 35 & $108-90-7$ & $4.56 \mathrm{E}+00$ & $7.10 \mathrm{E}+07$ & $6.42 \mathrm{E}-08$ & $\mathrm{~F}$ & Fish & 67 \\
\hline 36 & $110-54-3$ & $6.04 \mathrm{E}+03$ & $9.90 \mathrm{E}+06$ & $6.10 \mathrm{E}-04$ & $\mathrm{D}$ & Fish & 41 \\
\hline 37 & $110-82-7$ & $1.64 \mathrm{E}+03$ & $1.82 \mathrm{E}+07$ & $9.02 \mathrm{E}-05$ & $\mathrm{E}$ & Fish & 45 \\
\hline 38 & $111-42-2$ & $3.55 \mathrm{E}+02$ & $4.11 \mathrm{E}+04$ & $8.62 \mathrm{E}-03$ & $\mathrm{D}$ & Foliage vegetation & 32 \\
\hline 39 & $115-07-1$ & $1.11 \mathrm{E}+03$ & $4.66 \mathrm{E}+08$ & $2.39 \mathrm{E}-06$ & E & Fish & 60 \\
\hline 40 & $117-81-7$ & $8.02 \mathrm{E}+01$ & $1.36 \mathrm{E}+03$ & $5.91 \mathrm{E}-02$ & $\mathrm{C}$ & Aquatic mammal & 28 \\
\hline 41 & $118-74-1$ & $2.95 \mathrm{E}-02$ & $2.06 \mathrm{E}+03$ & $1.43 \mathrm{E}-05$ & $\mathrm{E}$ & Aquatic mammal & 54 \\
\hline 42 & $120-12-7$ & $1.22 \mathrm{E}+01$ & $1.04 \mathrm{E}+01$ & $1.17 \mathrm{E}+00$ & $\mathrm{~B}$ & Aquatic mammal & 16 \\
\hline 43 & $120-83-2$ & $3.16 \mathrm{E}+01$ & $1.22 \mathrm{E}+04$ & $2.60 \mathrm{E}-03$ & $\mathrm{D}$ & Aquatic mammal & 37 \\
\hline 44 & $124-18-5$ & $3.16 \mathrm{E}+01$ & $6.07 \mathrm{E}+05$ & $5.21 \mathrm{E}-05$ & $\mathrm{E}$ & Fish & 48 \\
\hline 45 & $127-18-4$ & $4.21 \mathrm{E}+01$ & $2.51 \mathrm{E}+07$ & $1.68 \mathrm{E}-06$ & $\mathrm{E}$ & Fish & 62 \\
\hline 46 & $129-00-0$ & $9.37 \mathrm{E}+01$ & $2.81 \mathrm{E}-01$ & $3.33 \mathrm{E}+02$ & $\mathrm{~A}$ & Aquatic mammal & 4 \\
\hline 47 & $603-35-0$ & $3.16 \mathrm{E}+01$ & $1.47 \mathrm{E}+03$ & $2.15 \mathrm{E}-02$ & $\mathrm{C}$ & Aquatic mammal & 30 \\
\hline
\end{tabular}




\begin{tabular}{|c|c|c|c|c|c|c|c|}
\hline ID \# & CAS \# & $\begin{array}{c}\mathbf{E}_{\mathrm{A}} \\
\mathrm{t} / \text { year }\end{array}$ & $\begin{array}{c}\text { L III-AWS E } \\
\text { t / year }\end{array}$ & $\begin{array}{c}\mathbf{R A F} \\
\mathbf{E}_{\mathbf{A}} / \mathbf{E}_{\mathbf{C}}\end{array}$ & $\begin{array}{c}\text { Risk ID } \\
\text { Bin }\end{array}$ & Media of concern & $\begin{array}{c}\text { Relative } \\
\text { Rank }\end{array}$ \\
\hline 48 & $608-93-5$ & $3.16 \mathrm{E}-01$ & $6.66 \mathrm{E}+03$ & $4.75 \mathrm{E}-05$ & $\mathrm{E}$ & Aquatic mammal & 49 \\
\hline 49 & $1163-19-5$ & $5.77 \mathrm{E}+00$ & $6.73 \mathrm{E}+01$ & $8.58 \mathrm{E}-02$ & $\mathrm{C}$ & Terrestrial carnivore & 27 \\
\hline 50 & $1222-05-5$ & $3.16 \mathrm{E}+01$ & $3.29 \mathrm{E}+02$ & $9.60 \mathrm{E}-02$ & $\mathrm{C}$ & Aquatic mammal & 26 \\
\hline 51 & $1330-78-5$ & $3.16 \mathrm{E}+03$ & $1.02 \mathrm{E}+03$ & $3.10 \mathrm{E}+00$ & $\mathrm{~B}$ & Aquatic mammal & 15 \\
\hline 52 & $1582-09-8$ & $3.16 \mathrm{E}-01$ & $6.88 \mathrm{E}+01$ & $4.59 \mathrm{E}-03$ & $\mathrm{D}$ & Aquatic mammal & 34 \\
\hline 53 & $1897-45-6$ & $3.16 \mathrm{E}+01$ & $4.45 \mathrm{E}-01$ & $7.11 \mathrm{E}+01$ & $\mathrm{~B}$ & Terrestrial carnivore & 7 \\
\hline 54 & $1912-24-9$ & $3.16 \mathrm{E}+01$ & $1.21 \mathrm{E}-02$ & $2.61 \mathrm{E}+03$ & A & Foliage vegetation & 1 \\
\hline 55 & $3380-34-5$ & $3.16 \mathrm{E}+01$ & $2.75 \mathrm{E}-01$ & $1.15 \mathrm{E}+02$ & A & Terrestrial carnivore & 6 \\
\hline 56 & $3846-71-7$ & $3.16 \mathrm{E}+01$ & $4.07 \mathrm{E}+01$ & $7.76 \mathrm{E}-01$ & $\mathrm{C}$ & Terrestrial carnivore & 17 \\
\hline 57 & $4979-32-2$ & $3.16 \mathrm{E}+01$ & $3.06 \mathrm{E}+03$ & $1.03 \mathrm{E}-02$ & $\mathrm{C}$ & Terrestrial carnivore & 31 \\
\hline 58 & $5285-60-9$ & $3.16 \mathrm{E}+03$ & $9.08 \mathrm{E}+02$ & $3.48 \mathrm{E}+00$ & $\mathrm{~B}$ & Aquatic mammal & 14 \\
\hline 59 & $6386-38-5$ & $3.16 \mathrm{E}+01$ & $1.35 \mathrm{E}+03$ & $2.34 \mathrm{E}-02$ & $\mathrm{C}$ & Terrestrial carnivore & 29 \\
\hline 60 & $13560-89-9$ & $3.16 \mathrm{E}+01$ & $2.17 \mathrm{E}+02$ & $1.46 \mathrm{E}-01$ & $\mathrm{C}$ & Terrestrial carnivore & 23 \\
\hline 61 & $15646-96-5$ & $3.16 \mathrm{E}+01$ & $2.20 \mathrm{E}+05$ & $1.43 \mathrm{E}-04$ & $\mathrm{D}$ & Aquatic mammal & 44 \\
\hline 62 & $23593-75-1$ & $3.16 \mathrm{E}-01$ & $1.23 \mathrm{E}+02$ & $2.57 \mathrm{E}-03$ & $\mathrm{D}$ & Terrestrial carnivore & 38 \\
\hline 63 & $25973-55-1$ & $3.16 \mathrm{E}+01$ & $4.67 \mathrm{E}+01$ & $6.77 \mathrm{E}-01$ & $\mathrm{C}$ & Terrestrial carnivore & 18 \\
\hline 64 & $26140-60-3$ & $3.16 \mathrm{E}+01$ & $1.62 \mathrm{E}+02$ & $1.95 \mathrm{E}-01$ & $\mathrm{C}$ & Terrestrial carnivore & 22 \\
\hline 65 & $32534-81-9$ & $3.16 \mathrm{E}+01$ & $2.42 \mathrm{E}+02$ & $1.30 \mathrm{E}-01$ & $\mathrm{C}$ & Aquatic mammal & 24 \\
\hline 66 & $32536-52-0$ & $3.16 \mathrm{E}+03$ & $5.74 \mathrm{E}+01$ & $5.51 \mathrm{E}+01$ & $\mathrm{~B}$ & Terrestrial carnivore & 9 \\
\hline 67 & $54079-53-7$ & $3.16 \mathrm{E}+01$ & $5.66 \mathrm{E}+01$ & $5.58 \mathrm{E}-01$ & $\mathrm{C}$ & Terrestrial carnivore & 19 \\
\hline 68 & $54827-17-7$ & $3.16 \mathrm{E}-01$ & $1.28 \mathrm{E}+04$ & $2.48 \mathrm{E}-05$ & E & Human & 51 \\
\hline 69 & $63936-56-1$ & $3.16 \mathrm{E}+03$ & $6.18 \mathrm{E}+01$ & $5.11 \mathrm{E}+01$ & $\mathrm{~B}$ & Terrestrial carnivore & 10 \\
\hline 70 & 89347-09-1 & $3.16 \mathrm{E}+03$ & $7.92 \mathrm{E}+02$ & $3.99 \mathrm{E}+00$ & $\mathrm{~B}$ & Aquatic mammal & 13 \\
\hline
\end{tabular}


RAIDAR - ES\&T Supporting Information (es0514085)

\section{References}

1. Mackay D. (2001), Multimedia Environmental Models: The Fugacity Approach Second Edition, Lewis Publishers, Boca Raton, Fla.

2. Mackay D.; Di Guardo A.; Paterson S.; Cowan C. Evaluating the environmental fate of a variety of types of chemicals using the EQC model. Environ. Toxicol. Chem. 1996, 15, 1627-1637.

3. Kelly B.C.; Gobas F.A.P.C.; McLachlan M.S. Intestinal absorption and biomagnification of organic contaminants in fish, wildlife, and humans. Environ. Toxicol. Chem. 2004, 23, 2324-2336.

4. Arnot J.A.; Gobas F.A.P.C. A food web bioaccumulation model for organic chemicals in aquatic ecosystems. Environ. Toxicol. Chem. 2004, 23, 2343-2355.

5. Kelly B.C.; Gobas F.A.P.C. An arctic terrestrial food-chain bioaccumulation model for persistent organic pollutants. Environ. Sci. Technol. 2003, 37, 29662974.

6. Hung H.; Mackay D. A novel and simple model of the uptake of organic chemicals by vegetation from air and soil. Chemosphere 1997, 35, 959-977.

7. U.S. EPA (1993), Wildlife Exposure Factors Handbook. U.S. Environmental Protection Agency.

8. Gobas F.A.P.C.; Mackay D. Dynamics of hydrophobic organic chemical bioconcentration in fish. Environ. Toxicol. Chem. 1987, 6, 495-504.

9. Czub G.; McLachlan M.S. A food chain model to predict the levels of lipophilic organic contaminants in humans. Environ. Toxicol. Chem. 2004, 23, 2356-2366.

10. McLachlan M.S. Bioaccumulation of hydrophobic chemicals in agricultural food chains. Environ. Sci. Technol. 1996, 30, 252-259.

11. Drouillard K.G.; Norstrom R.J. Dietary absorption efficiencies and toxicokinetics of polychlorinated biphenyls in ring doves following exposure to Aroclor mixtures. Environ. Toxicol. Chem. 2000, 19, 2707-2714.

12. Gobas F.A.P.C.; Kelly B.C.; Arnot J.A. Quantitative Structure-Activity Relationships for predicting the bioaccumulation of POPs in terrestrial food webs. QSAR Comb. Sci. 2003, 22, 329-336.

13. Beyer A.; Mackay D.; Matthies M.; Wania F.; Webster E. Assessing long-range transport potential of persistent organic pollutants. Environ. Sci. Technol. 2000, 34, 699-703.

14. Environment Canada. National Pollutant Release Inventory (2003). April 13, 2005, http://www.ec.gc.ca/pdb/npri/npri_home_e.cfm.U.S.

15. Environment Canada. Domestic Substances List - Preliminary Ecological Categorization Decisions CD-ROM, April 2005.

16. EPA (1996), Exposure Factors Handbook. U.S. Environmental Protection Agency.

17. U.S. EPA. EPI (Estimation Programs Interface) Suite version 3.12, November 30, 2004, http://www.epa.gov/oppt/exposure/docs/episuitedl.htm. 